Portland State University

PDXScholar

$1-1-2010$

\title{
Symmetric Near-Field Probe Design and Comparison to Asymmetric Probes
}

Jeffrey Jon Doughty

Portland State University

Follow this and additional works at: https://pdxscholar.library.pdx.edu/open_access_etds

Let us know how access to this document benefits you.

\section{Recommended Citation}

Doughty, Jeffrey Jon, "Symmetric Near-Field Probe Design and Comparison to Asymmetric Probes" (2010). Dissertations and Theses. Paper 390.

https://doi.org/10.15760/etd.390

This Thesis is brought to you for free and open access. It has been accepted for inclusion in Dissertations and Theses by an authorized administrator of PDXScholar. Please contact us if we can make this document more accessible: pdxscholar@pdx.edu. 
Near-Field Probe Design: A Comparison of Symmetric and Asymmetric Probes

\author{
by \\ Jeffrey Jon Doughty
}

A thesis submitted in partial fulfillment of the requirements for the degree of

\author{
Master of Science \\ in \\ Physics
}

Thesis Committee:

Erik Sánchez, Chair

John Freeouf

Andres La Rosa

Portland State University

(C)2010 


\begin{abstract}
Tip Enhanced Near-field Optical Microscopy (TENOM) is a method for optically imaging at resolutions far below the diffraction limit. This technique requires optical nano-probes with very specialized geometries, in order to obtain large, localized enhancements of the electromagnetic field, which is the driver behind this imaging method. Traditional methods for the fabrication of these nano-probes involve electrochemical etching and subsequent FIB milling. However, this milling process is non-trivial, requiring multiple cuts on each probe. This requires multiple rotations of the probe within the FIB system, which may not be possible in all systems, meaning the sample must be removed from vacuum, rotated by hand and placed back under vacuum. This is time consuming and costly and presents a problem with reproducibility. The method presented here is to replace multiple cuts from a side profile with a small number of cuts from a top down profile. This method uses the inherent imaging characteristics of the FIB, by assigning beam dwell times to specific locations on the sample, through the use of bitmap images. These bitmaps are placed over the sample while imaging and provide a lookup table for the beam while milling. These images are grayscale with the color of each pixel representing the dwell time at that pixel. This technique, combined with grayscale gradients, can provide probes with a symmetric geometry, making the system polarization independent.
\end{abstract}




\section{Dedication}

This work is dedicated to every person who never has no in their heart. Those people who face all odds and persevere. You are the people who make the world turn. Never give up, your dreams can indeed be realized.

Specifically, I'd like to thank my wife Jamie, my daughter Olivia, and Eleanor Wentz for their support and love. I would not be where I am today without them. 


\section{Acknowledgments}

There are a number of people who have helped me throughout this first foray into research. The obvious first would be my advisor, Dr. Erik Sánchez, who through his patience and sense of humor has helped to form me into a researcher, despite my endless stream of questions. This guidance is priceless and the time taken from his busy schedule to mentor me will never be forgotten. It is a privilege to work for someone with such an enormous skill set and such a drive to succeed. This experience has changed my life forever.

Next, I think it is very appropriate to thank the members of my defense committee, Dr. John Freeouf and Dr. Andres La Rosa. For their patience throughout this process, with all my rescheduling I appreciate their help greatly.

I would also like to thank Dr. Derek Nowak for his help. Derek is one of those amazing people, like Erik Sánchez, who is extremely competent and is willing to share his knowledge to bring others to his level. Derek has a way of inspiring that I've never seen before and I am in his debt for helping me stay motivated, as well as actually helping me accomplish my goals.

My lab mates, Andrew Lawrence and Mike DeArmond, you guys are awesome. In addition to the help you were always willing to give with my project; to have friends for coworkers makes every difference in the world when coming to the 
lab every day for years on end. Also Mike, thank you for doing all of the grammar and spelling end of the proofreading, that help was invaluable.

Kelvin Pittman I would like to thank for his proofreading skills. Thanks also go to Philip Witham for his suggestions on different directions to take when running into problems. Many times a fresh perspective is extremely helpful. I'd also like to thank Amber Lauer for being my nemesis and for her occasional computer help.

Finally, I'd like to thank Free Geek for their generous donation of 11 modeling computers. I can't even imagine completing this project without this resource. This donation saved me an uncountable number of hours. Thanks, solely to this donation, I was able to run 13 models at any given time. Many of these models would take 5 days to run. I would still be running models were it not for them. 


\section{Table of Contents}

Abstract

Dedication $\quad$ ii

Acknowledgments iii

List of Figures vi vi

I. Introduction $\mathbf{1}$

1.1: Diffraction Limit $\quad 1$

1.2: Near Field Microscopy 3

1.3: Specific geometries/Motivation $\quad 6$

II. Introduction to modeling $\quad \mathbf{1 1}$

2.1: Introduction to FDTD $\quad 11$

2.2: The Incident and Scattered Fields $\quad 15$

2.3: Material Parameters $\quad 17$

2.4: Realistic Modeling Spaces - Limitations of FDTD 18

III. Modeling of Probes 25

3.1: Varying location of focal volume and polarization independence 25

IV. $\quad$ Fabrication Methods $\quad 48$

4.1: Electrochemical Etching $\quad \mathbf{4 8}$

4.2: Focused Ion Beam Systems

4.3: Stream Files $\quad 54$

4.4: Automation through LabVIEW ${ }^{\mathrm{TM}}$

4.5: Pixmaps $\quad 57$

4.6: Creating Stream Files from Bitmaps $\quad 58$

4.7: Bitmap Geometries Used 62

4.8: Experimental Procedures $\quad 63$

$\begin{array}{lll}\text { V. Discussion and Conclusions } & 69\end{array}$

5.1: Discussion and Conclusions $\quad 69$

$\begin{array}{lll}\text { VI. } & \text { References } & 74\end{array}$ 


\section{List of Figures}

\section{Chapter I: Introduction}

1.1: Light focused through a lens, illustrating the concepts of numerical aperture and focal volume.

1.2: The idea behind TENOM. Light is incident from below a metal probe in such a way as to produce a strong localized field at the tip apex.

1.3: Concepts of side illumination, showing the polarization requirement.

1.4: An asymmetric TENOM probe. This design allows for both topographic and optical imaging.

1.5: The asymmetric tip fabrication process. A) Pre-FIBed tip. B) First cut. C) $180^{\circ}$ rotation. D) Second cut. E) $180^{\circ}$ rotation. F) Third cut. G) $180^{\circ}$ rotation. H) Fourth and final cut.

\section{Chapter II: Introduction to modeling}

2.1: A Yee cell, in which is calculated the electric field at each edge and the magnetic field at each face.

2.2: The spherical coordinate system for xFDTD.

2.3: A $100 \mathrm{~nm}$ radius sphere approximated by $80 \mathrm{~nm}$ cubes.

2.4: An $80 \mathrm{~nm}$ cell size with $1 \mathrm{~nm}$ submeshing for the sphere.

2.5: Zoomed in on the submeshed region from Figure 2.4.

2.6: Partial submeshing of a sphere.

2.7: It takes roughly 5 microns to transition from $1 \mathrm{~nm}$ cells to $80 \mathrm{~nm}$ cells.

\section{Chapter III: Modeling of Probes}

3.1: The focal volume placement in the xy-plane. 
3.2: Lens with index of refraction equal to $n=1.541$. A NA of $\sim 1.18$ is calculated. 27

3.3: The scale used in the following figures.

3.4: The yz-plane field response for an asymmetric probe, with polarization in the $y$ direction.

3.5: The xy-plane field response for an asymmetric probe with polarization in the $y$ direction.

3.6: The yz-plane field response for an asymmetric probe, with polarization in the $\mathrm{z}$ direction.

3.7: The xy-plane field response for an asymmetric probe, with polarization in the $\mathrm{z}$ direction.

3.8: A sharp tip and its field response.

3.9: The yz-plane field response for a y polarization at point A in Figure3.1.

3.10: The xy-plane field response for a y polarization at point A in Figure 3.1.

3.11: The yz-plane field response for the second half of the wavelength at point $\mathrm{A}$ in Figure 3.1. The polarization is in $\mathrm{y}$.

3.12: The xy-plane field response for the second half of the wavelength at point $\mathrm{A}$ in Figure 3.1. The polarization is in $\mathrm{y}$.

3.13: The yz-plane field response for a z polarization at point A in Figure 3.1.

3.14: The xy-plane field response for a z polarization at point A in Figure 3.1.

3.15: The yz-plane field response for a y polarization at point B in Figure 3.1.

3.16: The xy-plane field response for a y polarization at point B in Figure 3.1.

3.17: The yz-plane field response for a y polarization at point B in Figure 3.1.

3.18: The xy-plane field response for a z polarization at point B in Figure 3.1.

3.19: The yz-plane field response for a y polarization at point $\mathrm{C}$ in Figure 3.1. 
3.21: The xy-plane field response for a y polarization at point D in Figure 3.1.

3.22: The yz-plane field response for a $\mathrm{z}$ polarization at point $\mathrm{D}$ in Figure 3.1.

3.23: The xy-plane field response for a z polarization at point D in Figure 3.1.

3.24: The yz-plane field response for a y polarization at point $\mathrm{E}$ in Figure 3.1.

3.25: The xy-plane field response for a y polarization at point $\mathrm{E}$ in Figure 3.1.

3.26: The yz-plane field response for a z polarization at point E in Figure 3.1.

3.27: The xy-plane field response for a z polarization at point E in Figure 3.1.

\section{Chapter IV: Fabrication Methods:}

4.1: A sharp tip, post etch.

4.2: Focused Ion Beam interaction volume.

4.3: The white square shows an area of damage from imaging with the FIB.

4.4: An example of a stream file.

4.5: An example VI, including both block diagram (left) and front panel (right).

4.6: The process by which the LabVIEW VI converts a bitmap into a stream file.

4.7: A four pixel bitmap and its corresponding stream file.

4.8: The graphical user interface for the VI.

4.9: The two bitmaps used in the fabrication process.

4.10: The etch circuit used in the electrochemical etching step of the fabrication process.

4.11: Proof of concept, however, milled off center and with the wrong bitmap. The tip inside the hole is $\sim 500 \mathrm{~nm}$ in diameter. This is very preliminary. 


\section{Chapter V: Discussion and Conclusions:}

5.1: Zoomed out head on view of a tip that was fabricated using the lookup table method.

5.2: A top down view of the best tip created using this lookup table method.

Zoomed in view of the damaged square in Figure 5.1.

5.3: A side view of the tip shown in Figures 5.1 and 5.2. 


\section{Chapter 1: Introduction}

\section{1: Diffraction Limit}

The diffraction limit has traditionally been the way of describing the best possible resolution that a given optical system can obtain, with perfect conditions (monochromatic light, aberration free lenses, highest possible numerical aperture). Specifically, it is defined by the use of equation 1.1 (known as the Rayleigh criterion) and 1.3 as the closest distance two objects can be from one another and remain distinguishable as two objects. This relationship is dependant on $\lambda$, the wavelength of the incident light, and the numerical aperture of the lens system, which is defined as the product of the index of refraction (n) of the medium through which the light is propagating after the lens and the sine of the half angle $\alpha$, as shown in Figure 1.1. The numerical aperture is a way of representing how tightly light is focused through a lens. The Rayleigh criterion predicts that the spot size is given by:

$$
d \cong 1.22 \frac{\lambda}{2 \cdot N A}
$$

where $N A=n \cdot \sin \alpha$ is the numerical aperture. The final distance two objects can be to each other and still be resolvable as:

$$
d \cong 1.22 \frac{\lambda}{2 \cdot n \cdot \sin \alpha}
$$




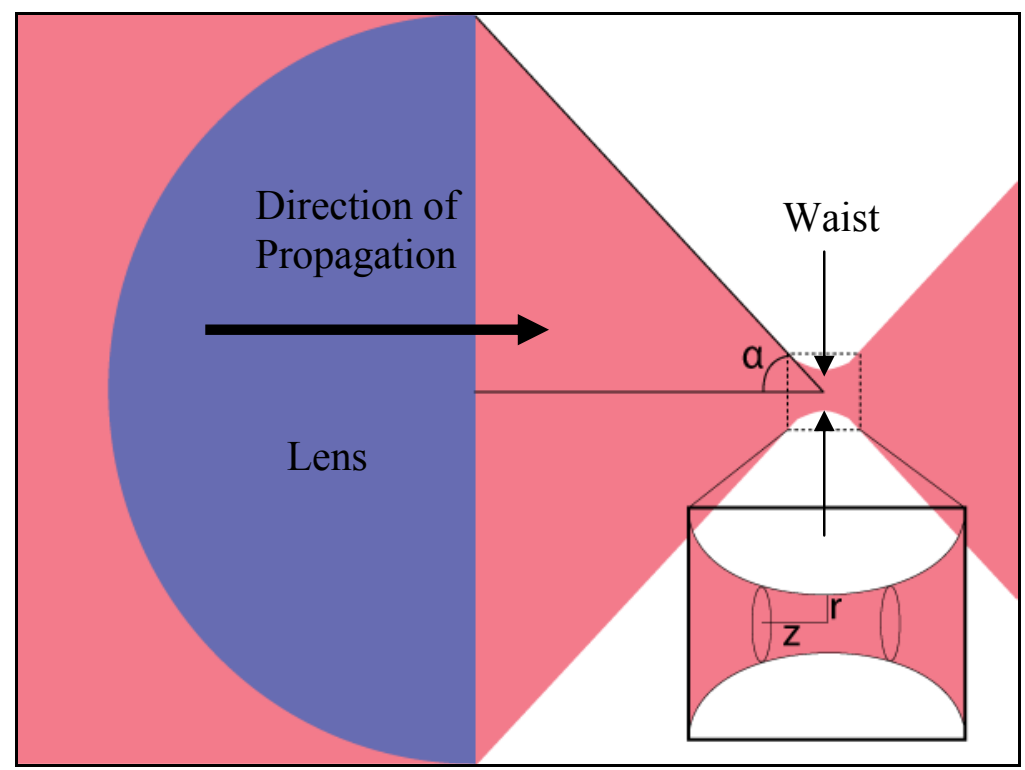

Figure 1.1: Light focused through a lens, illustrating the concepts of numerical aperture and focal volume.

From equations $1.1-1.2$, it can be elucidated that to achieve a high resolution, or low d value, a short wavelength of light must be used. Second, a high index of refraction will also give a higher resolution. For example, for a very tightly focused beam $\left(80^{\circ}\right)$ of $800 \mathrm{~nm}$ light focused in air $(n=1.0003) d$ roughly equals $495 \mathrm{~nm}$. In order to achieve a high numerical aperture, the lens must be coupled to an index higher than that of air, such as oil, which is then in contact with the glass cover slip which the sample is deposited on. The oil and substrate material (the cover slip) need to have matched indices of refraction, or losses will occur at the interface between them, in the form of reflection and a subsequent loss in image quality. With an oil/glass index of refraction equal to $n=1.5$, and the same parameters as above, a resolution of $\sim 349 \mathrm{~nm}$ is achieved, which is still very large for many applications.

Placing the concept of spot size into a 3 dimensional picture gives a focal volume, which is generally approximated as a cylinder. This is the region at which the lens focuses the light most intensely. This focal volume is explained in terms of the 
beam waist, or $r$ in Figure 1.1 and the Rayleigh range, or $z$ in Figure 1.1. The Rayleigh length is directly related to the spot size and wavelength as dictated by equation 1.4:

$$
z=\frac{\pi r^{2}}{\lambda}
$$

which for a spot of $800 \mathrm{~nm}$ light, with a radius of $250 \mathrm{~nm}$, the Rayleigh range is roughly $245 \mathrm{~nm}$.

\section{2: Near-field Microscopy}

In 1928, E. H. Synge proposed the concept of near field excitation in a microscope in order to break the diffraction limit set forth by the Rayleigh criterion [Synge, 1928]. The idea used light incident on one side of an opaque material with a very small aperture, situated very close (on the order of one wavelength of the source) to the sample to achieve a spot size smaller than the diffraction limit. This spot is then scanned across the sample to be imaged, with the light collected at each point by a sensor. Unfortunately, in 1928 the ability to experimentally achieve what Synge proposed was not realistic, due to technological limitations at the time. Synge's ideas were forgotten until Bethe in 1944, Bouwkamp in 1950 and O'Keefe published similar work in 1956 [Bethe, 1944]; [Bouwkamp, 1950]; [O’Keefe, 1956]. In his paper, O'Keefe even mentions the lack of feasibility for the theory to be realized in any short time scale. The experiment did not catch up to this early theoretical work until Ash and Nichols obtained near field images in 1972 using light in the microwave regime, incident on a $1.5 \mathrm{~mm}$ aperture [Ash and Nichols, 1972]. 
The first images with better than diffraction limited resolution in the visible regime were in 1984, by Pohl et al, with Lewis et al publishing within the same year [Pohl, Denk, and Lanz, 1984]; [Lewis et al, 1984]. In 1987, the experimental capabilities of near field microscopy were realized using a different probe design by Eric Betzig [Betzig, 1987]; [Betzig, 1992]; [Betzig, 1993]. Betzig utilized the concept of a tapered, metal-coated glass pipette acting as a waveguide. As the pipette gets smaller, all modes of transmission are cutoff and the field is transported through the metal coating. At the end of the fiber, the metal-fiber interface reradiates the field annularly with a ring the size of the end radius of the metal/pipette interface.

However, this technique (NSOM) does not achieve the resolutions desirable to many applications. This is due to the fact that the end radius of the fiber or pipette is generally rather large. The ability to create an apertured near field probe with ideal parameters is so far not possible. It would be necessary to create a very small end radius with a low aspect ratio, because once the taper becomes such a width that real transmission modes are no longer supported, the evanescent wave will decay quickly. There are various other complications with the apertured near-field approach; such as difficulty in obtaining a perfect field confining metal coating on the fiber and insufficient topographic imaging capabilities due to the coating.

In 1985, John Wessel published a paper outlining the concept of using a submicron sized metal particle as a probe for near field microscopy [Wessel, 1985]. This brought about a revolution in near field imaging, when researchers realized that it was possible to get higher resolutions with an apertureless near-field scanning optical microscope (ANSOM) than was possible with apertured versions. Since 1985, 
multiple groups have utilized metal tips illuminated by scattering the field from a laser for near field imaging. The first to do so was Specht et al in 1992 followed closely by Kawata and Inouye, in 1995, both with symmetric metal tips [Specht et al, 1992]; [Kawata and Inouye, 1995]. Zenhausern et al were also within this time frame, obtaining 10 angstrom resolution [Zenhausern, Martin and Wickramasinghe, 1995]. Then the first design to utilize the concept of field enhancement rather than just scattering came along. TENOM, or Tip Enhanced Near-field Optical Microscopy, is a subset of ANSOM, in that it is apertureless; however TENOM utilizes an asymmetric geometry, as outlined by Sánchez et al in 1999 [Sanchez, Novotny, and Xie, 1999], with the current, unpublished version shown in Figure 1.2. In this image, $\sim 800 \mathrm{~nm}$ light is incident on a 1.4 NA objective lens, which focuses on the bulk region of the metallic tip (gold in this case). The polarization of the incident light in the y direction causes a high surface charge density in the metal, which causes the field to reradiate at the apex of the tip portion as a sub-diffraction limited spot. This acts as the sub-diffraction limited illumination source that the sample is scanned under. 


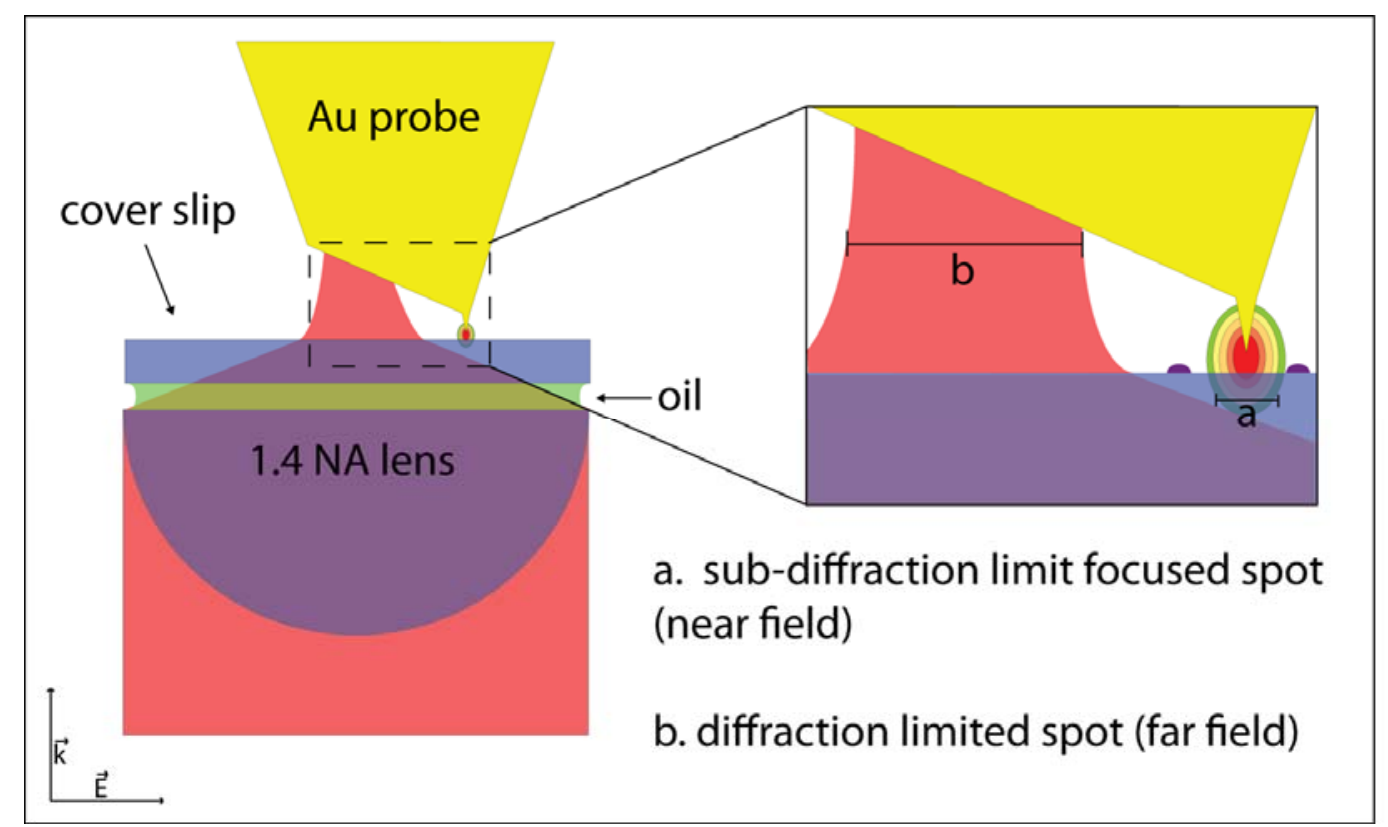

Figure 1.2: The idea behind TENOM. Light is incident from below a metal probe in such a way as to produce a strong localized field at the tip apex.

\section{3: Specific geometries / Motivation}

Very sharp tips alone will not produce ideal near field images with the TENOM design. This will be shown in subsequent chapters through Finite Difference Time Domain modeling. This is due to the positioning of the objective lens from below and the difficulty in achieving a $\mathrm{z}$ polarization (as seen in Figure 1.4) of the incident light. A very sharp tip could possibly work with a side illumination system as shown in Figure 1.3, but the requirement of the tip to be within nanometers of the objective lens (in order to maintain a high numerical aperture and thus a low power level) makes this idea extremely difficult in implementation. 


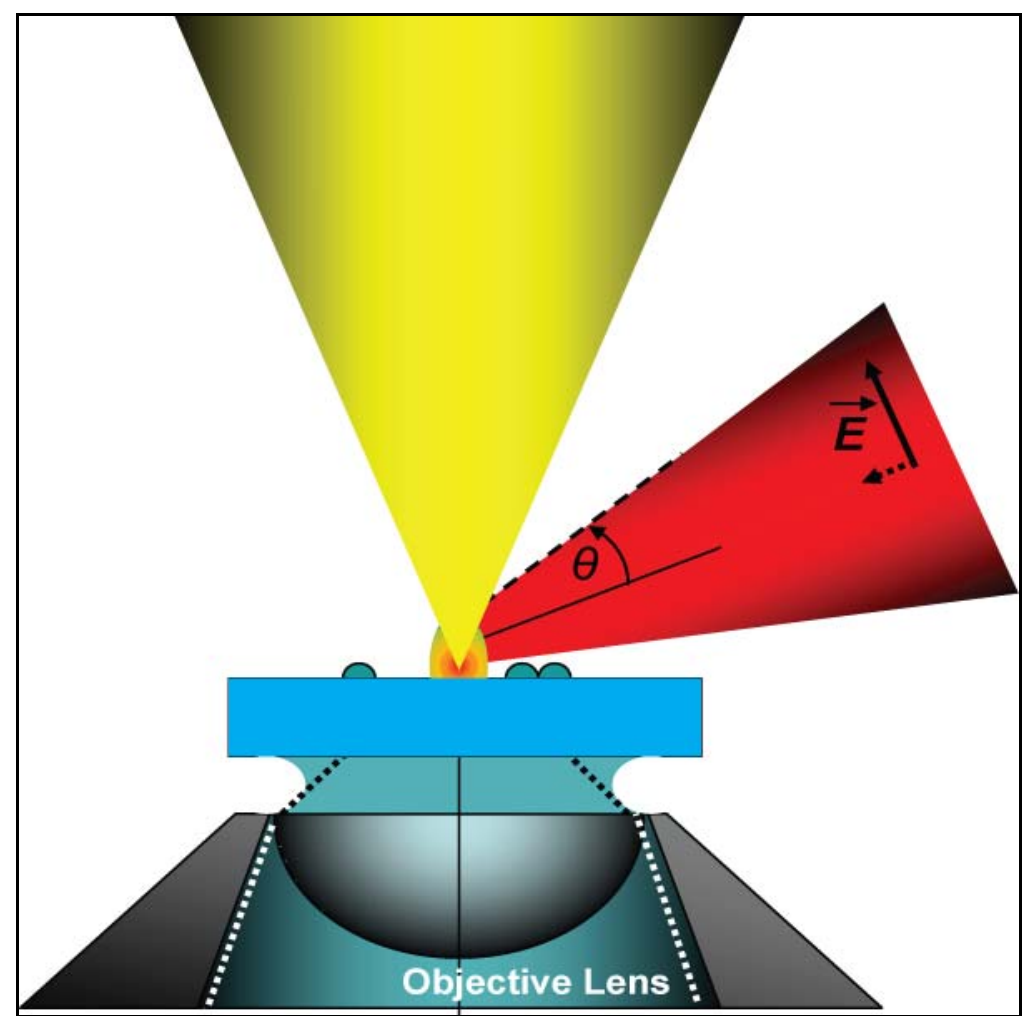

Figure 1.3: Concepts of side illumination, showing the polarization requirement.

Systems with a side illumination have been developed by Mehtani, however, these systems suffer from a need to use high laser powers, which can photobleach the sample and introduce high background levels, making it difficult to resolve small features [Mehtani, 2005]. By utilizing bottom illumination, it is possible to use power levels in the microwatt regime, as opposed to the milliwatt regime published by Mehtani. Therefore, more tailored geometries needed to be explored for a bottom illumination system.

There is another aspect to consider when thinking about geometries, and that is the fact that a TENOM system is not just an optical microscope, but a scanning probe microscope as well. TENOM makes use of shear force microscopy, which does need 
a sharp tip in order to produce topographies with high spatial resolutions. Therefore, the TENOM system needs a probe that is both sharp for topographic imaging, yet specifically tailored to the polarization of incident light, in order to achieve high field enhancements. Figure 1.4 shows a compromise between the two, giving both a good topography and good near field imaging.

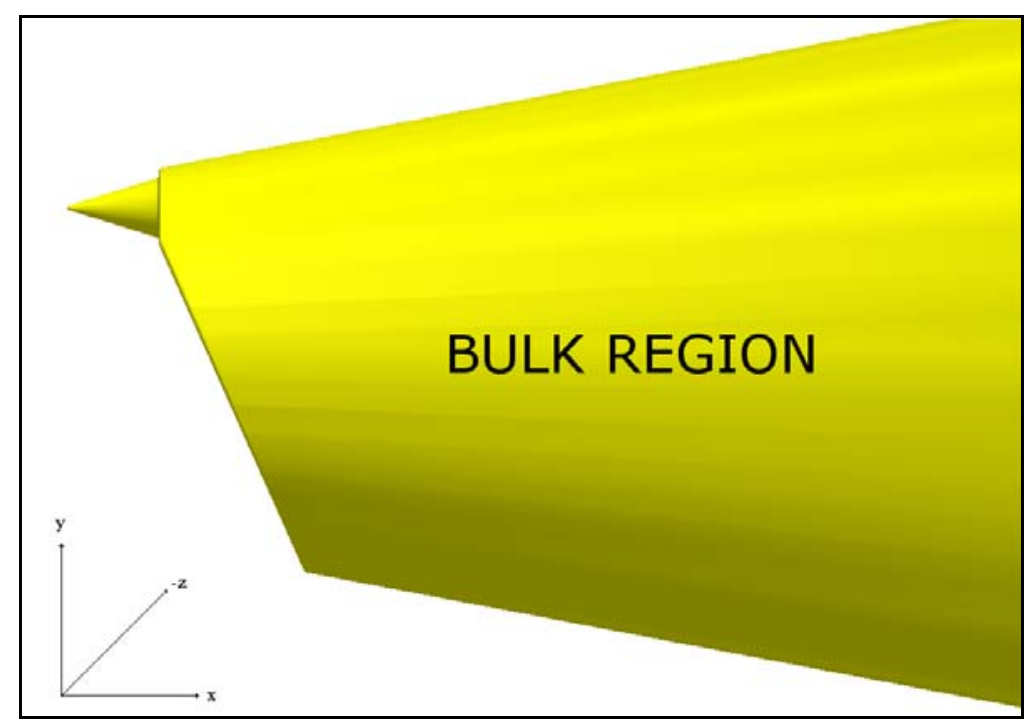

Figure 1.4: An asymmetric TENOM probe. This design allows for both topographic and optical imaging.

There are two difficulties with this geometry: the complications related to fabrication, and the necessity of a specific polarization in the xy-plane, as shown previously in Figure 1.2. To make this tip, multiple cuts and rotations must be made with a Focused Ion Beam system (FIB). Some of these rotations may not even be possible in certain FIB systems. Also, for every rotation and cut, there is a period of time associated with obtaining a good image (ie: stigmation and focus). This time is inversely proportional to tip apex radius, because the longer the ion or electron beam dwells on the tip, the blunter it becomes. This is due to the inherent milling effect 
associated with interaction volumes and charged particle microscopy. Figure 1.5 shows the steps required to create these asymmetric probes.

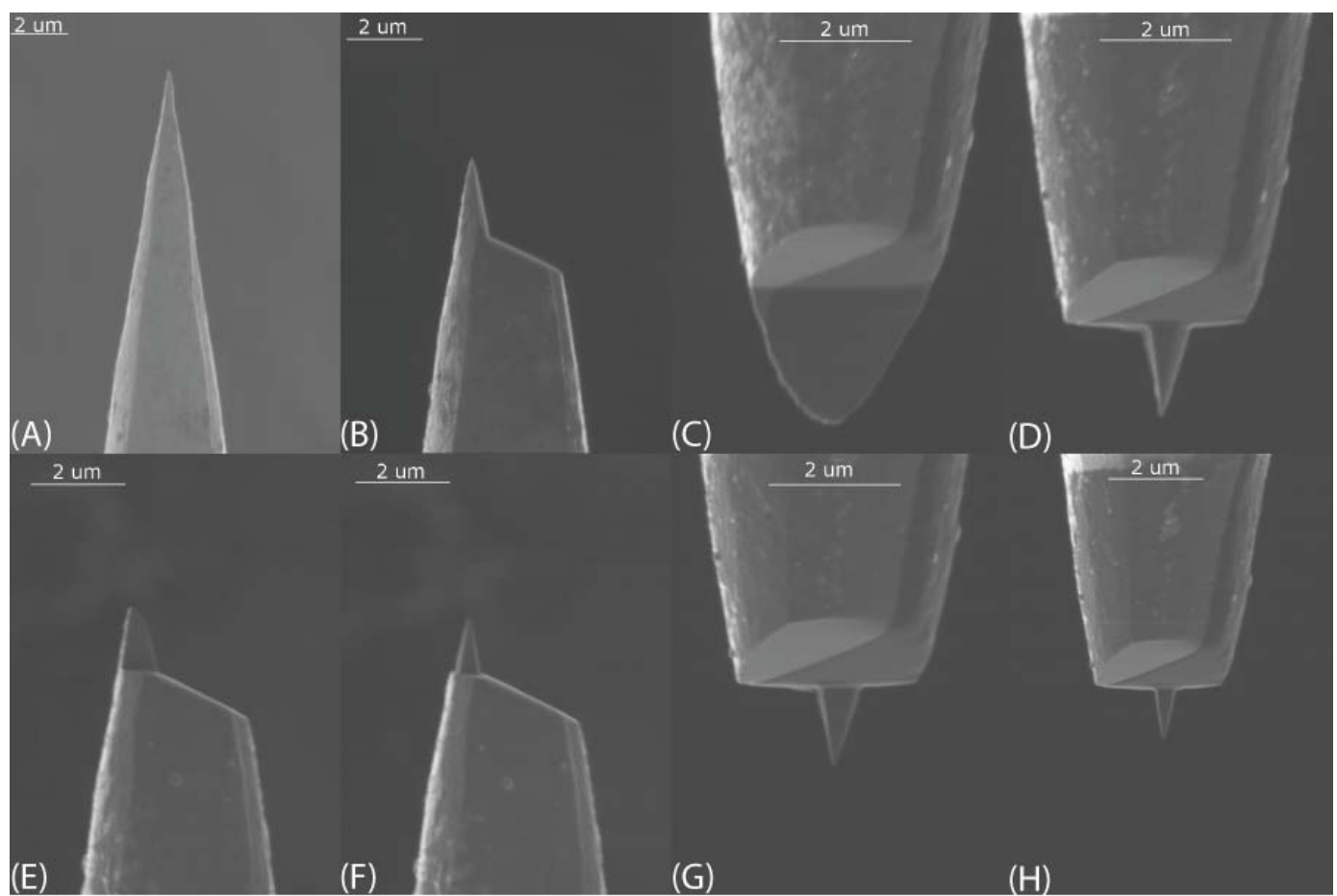

Figure 1.5: The asymmetric tip fabrication process. A) Pre-FIBed tip. B) First cut. C) $180^{\circ}$ rotation. D) Second cut. E) $180^{\circ}$ rotation. F) Third cut. G) $180^{\circ}$ rotation. H) Fourth and final cut.

The polarization dependence is not yet fully understood theoretically, however, the asymmetric tip design requires a polarization in the $\mathrm{x}$ axis, focused on the bulk region shown in Figure 1.4, in order to achieve a large field at the end of the tip. This has been shown empirically both through finite difference time domain modeling and through the effect of polarization on photon counts in a TENOM system. The FDTD results will be discussed at length in chapters 3 and 4 .

Due to the complexities in fabrication and the polarization dependence of the asymmetric tip design, a method for fabrication of a new symmetric design is outlined in chapter 5. This new design is a compromise between the easy fabrication of a sharp 
tip, with the good imaging capabilities of the asymmetric design, fabricated in a way that eliminates previous problems and provides for semi-autonomy, making it applicable for mass production. As an added benefit, polarization dependence is reduced, because the possibility of utilizing polarization in any direction in the xyplane is possible. 


\section{Chapter 2: Introduction to Modeling \\ 2.1: Introduction to FDTD Modeling}

Finite difference time domain (FDTD) modeling has been a useful technique of the numerical field modeling community. This is primarily due to the wide range of applications that FDTD can address and the relatively low computational requirements to run semi-realistic models. Unlike many modeling algorithms, FDTD operates in the time domain, rather than frequency, allowing the electric and magnetic fields to be calculated from Maxwell's equations in a time stepping manner. In many applications, including TENOM, modeling is the only way to qualify the field response as a function of geometry alone. It is known that high field intensity is needed very close to the sample in order to achieve good near field imaging with a technique like TENOM. This field can easily be examined in the modeling world, while it cannot in the physical world. In order to determine optimal geometries, it is not possible to make all the different physical probes and test them all quickly in the microscope, and obtain a result that isolates the geometry as the single variable. Therefore, modeling is a necessity for the development of suitable probe geometries in TENOM. For this purpose, Remcom's xFDTD, version 6.2, based on the formulism laid out by Kunz and Leubbers, was used extensively [Kunz and Leubbers, 1993].

The idea for FDTD came from a paper published in 1966 by Kane Yee [Yee,

1966]. In this paper, Yee outlines the concept of using Maxwell's equations (in Gaussian units):

$$
\vec{\nabla} \times \vec{E}=-\frac{\partial \vec{B}}{\partial t},
$$




$$
\begin{aligned}
& \vec{\nabla} \times \vec{H}=\frac{\partial \vec{D}}{\partial t}+\vec{J}, \\
& \vec{\nabla} \cdot \vec{D}-\rho=0, \\
& \vec{\nabla} \cdot \vec{B}=0
\end{aligned}
$$

where:

$$
\begin{aligned}
& \vec{D}=\varepsilon \vec{E}, \\
& \vec{B}=\mu \vec{H},
\end{aligned}
$$

in a finite difference form, to leapfrog solutions of $\vec{E}$ and $\vec{H}$ (the electric and magnetic fields) through an isotropic medium. This utilizes small cubes, later called Yee cells, to form the desired geometry. The electric field, $\vec{E}$, is calculated at every edge of the Yee cell, and the magnetic field, $\vec{H}$, is calculated at every face. A Yee cell is shown in Figure 2.1, in which it is to be noted that there is a slight offset of $1 / 2$ the cell size between the electric and magnetic fields. The size of the Yee cell determines the overall resolution of the field calculated and the spatial discrepancy between electric and magnetic fields. The number of cells determines the computational power required, as will be discussed in section 2.4. Basically, geometries are approximated by a number of cubes. As the physically represented size of the cubes decrease, the object is more accurately represented, but as the number of cubes increases which drastically increases the necessary computational power. 


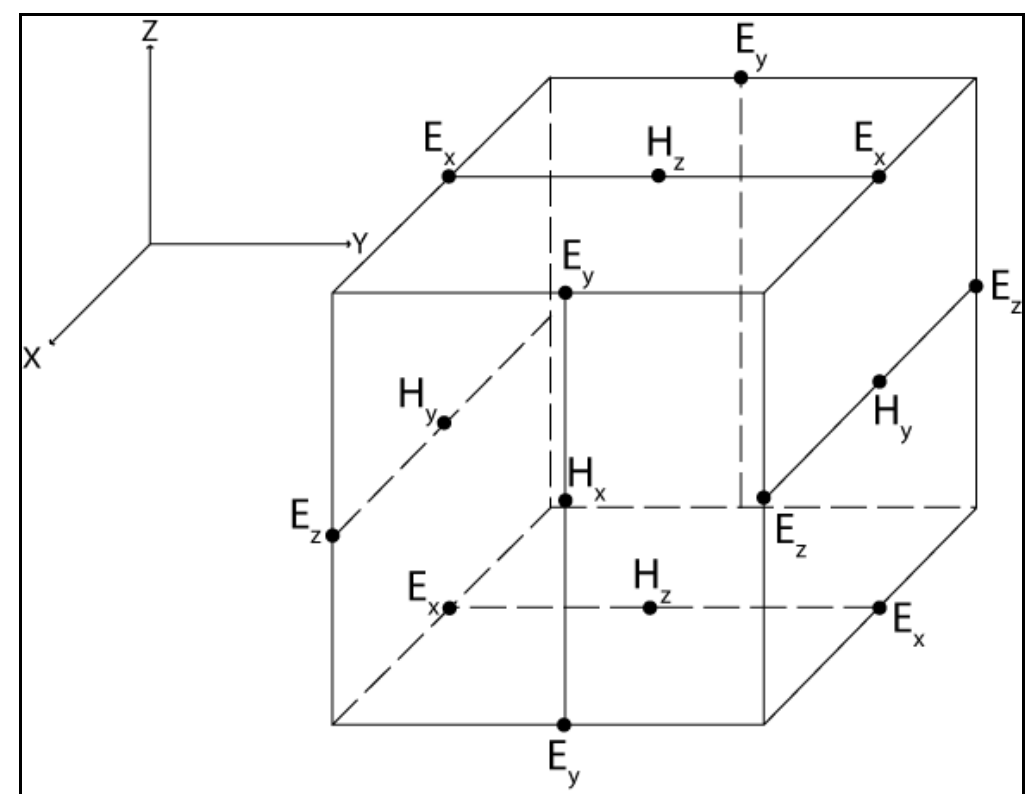

Figure 2.1: A diagram of a Yee cell, in which is calculated the electric field at each edge and the magnetic field at each face.

As outlined by Yee, there are some requirements for the cell size; it is not just an arbitrary value. It is necessary to have a cell size that is small enough that the fields remain roughly constant throughout the cell. This means that, conventionally, the cell size must be smaller than, $\lambda / 10$ where $\lambda$ is the wavelength of the incident field. This comes from an empirical usage of the Courant stability criterion and the Nyquist sampling theorem, which states that in order to have an adequate representation of a space, at least 2 samples must be taken per wavelength. The Courant stability criterion is:

$$
c \Delta t \leq \frac{1}{\sqrt{\frac{1}{(\Delta x)^{2}}+\frac{1}{(\Delta y)^{2}}+\frac{1}{(\Delta z)^{2}}}},
$$

where $\Delta x, \Delta y, \Delta z$ are the size of the edges of the Yee cell and $c$ is the speed of light. One thing to note is that $c$ may not represent the speed of light in a vacuum, depending on the medium the light is traveling through. For the Kunz and Luebbers formulism 
of FDTD, 10 samples per wavelength must be taken for reasons related to a variety of approximations, in addition to Courant stability and Nyquist sampling. This will also assure that the incident field does not travel through more than one cell at a time, which would also cause instabilities. In summary, the field must remain constant while travelling through each cell and may not travel through multiple cells in a single time step.

The basis of FDTD is in Maxwell's equations, which can be seen in equations 2.1 through 2.5. As outlined by Kunz and Leubbers on page 12, it is only necessary to consider 2.1 and 2.2, because by taking the divergence of the curl equations, we can obtain the relationship that the divergence equations 2.3 and 2.4 are constant, and since they begin at 0 , are equal to 0 for all time [Kunz and Leubbers, 1993]. Therefore, only discretized versions of the curl equations:

$$
\begin{aligned}
& \frac{\partial \vec{E}}{\partial t}=-\frac{\sigma}{\varepsilon} \vec{E}+\frac{1}{\varepsilon}(\vec{\nabla} \times \vec{H}), \\
& \frac{\partial \vec{H}}{\partial t}=-\frac{1}{\mu}(\vec{\nabla} \times \vec{E})-\frac{\sigma^{*}}{\mu} \vec{H},
\end{aligned}
$$

are used in the FDTD formulism. The derivation of equations 2.8 and 2.9 are straightforward, aside from the magnetic conductivity term in equation $2.9\left(\frac{\sigma^{*}}{\mu} \vec{H}\right)$. 


\section{2: The Incident and Scattered Fields}

The fields in XFDTD are specified in typical spherical coordinates, shown in Figure 2.2 .

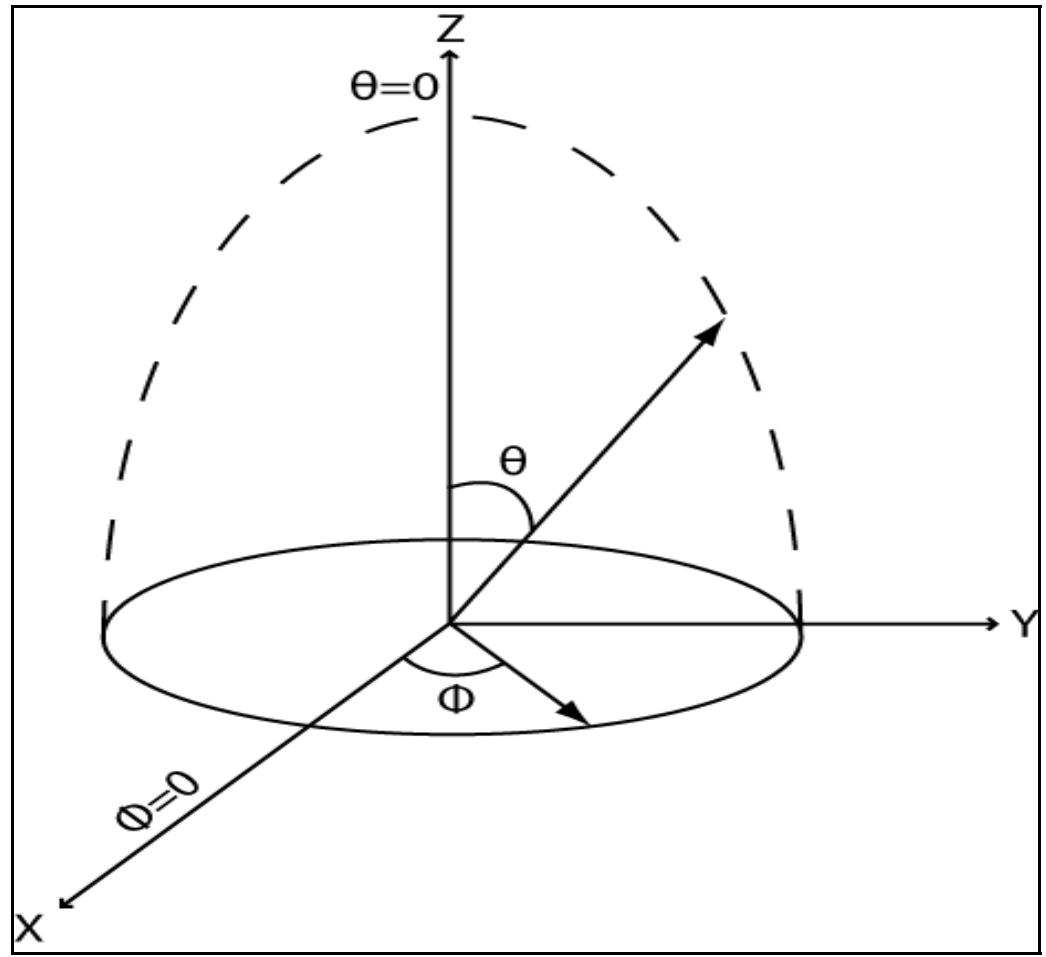

Figure 2.2: Diagram of the spherical coordinate system for xFDTD.

Only the electric field need be given, because the magnetic field is calculated from the electric in a similar manner. The field is specified through:

$$
\vec{E}=\left[E_{\theta} \hat{\theta}+E_{\phi} \hat{\phi}\right] f\left(t+\frac{\left(r^{\prime} \cdot \hat{r}\right)}{c}+\frac{R}{C}\right),
$$

where $E_{\theta}$ and $E_{\phi}$ are given by the user. The $f(t)$ allows for different types of signals to be used, such as Gaussian pulses, sinusoids, etc, $R$ is a variable which allows for propagation of the field to the object, rather than appearing instantly at it. The $\left(r^{\prime} \cdot \hat{r}\right)$ term determines which direction the field will propagate from. The Cartesian 
coordinates are readily obtained from the theta and phi components of the specified field, through the following:

$$
E_{x}=E_{\theta} \cos \theta \cos \phi-E_{\phi} \sin \phi,
$$

and

$$
E_{z}=-E_{\theta} \sin \theta,
$$

and

$$
E_{y}=E_{\theta} \cos \theta \sin \phi+E_{\phi} \cos \phi,
$$

are components for a typical transformation from spherical to Cartesian coordinate systems. Due to the incident field being specified by the user, only the field scattered off of objects need be calculated. This calculation, as previously mentioned, is done through a differencing of Maxwell's equations, through:

$$
\begin{aligned}
& \frac{\partial f}{\partial t} \approx \frac{f\left(x, t_{2}\right)-f\left(x, t_{1}\right)}{\Delta t}, \\
& \frac{\partial f}{\partial x} \approx \frac{f\left(x_{2}, t\right)-f\left(x_{1}, t\right)}{\Delta x} .
\end{aligned}
$$

where $\mathrm{f}$ is some function of time. From this relationship, it is possible to discretize equations 2.8 and 2.9 , although they must first be put into the component forms. The $\mathrm{x}$ component of equation 2.8 is shown in equation 2.16 :

$$
\frac{\partial E_{x}^{s}}{\partial t}=\frac{1}{\varepsilon_{0}}\left(\frac{\partial H_{z}^{s}}{\partial y}-\frac{\partial H_{y}^{s}}{\partial z}\right),
$$

where the $s$ denotes the scattered field. The total field is merely the sum of the incident and the scattered fields:

$$
E_{\text {total }}=E_{\text {scattered }}+E_{\text {incident }} \text {. }
$$


Using equations $2.14,2.15$, and 2.16 the $\mathrm{x}$ component of the electric field at each time step can be calculated:

$$
\begin{aligned}
& E_{x}^{s}(I, J, K)^{n}=E_{x}^{s}(I, J, K)^{n-1}\left(\frac{\varepsilon}{\varepsilon+\sigma \Delta t}\right) \\
& -\left(\frac{\sigma \Delta t}{\varepsilon+\sigma \Delta t}\right) E_{x}^{i}(I, J, K)^{n}-\left(\frac{\left(\varepsilon-\varepsilon_{0}\right) \Delta t}{\varepsilon+\sigma \Delta t}\right) \dot{E}_{x}^{i}(I, J, K)^{n} \\
& +\frac{H_{z}^{s}(I, J, K)^{n-\frac{1}{2}}-H_{z}^{s}(I, J-1, K)^{n-\frac{1}{2}}}{\Delta y}\left(\frac{\Delta t}{\varepsilon+\sigma \Delta t}\right) \\
& +\frac{H_{y}^{s}(I, J, K)^{n-\frac{1}{2}}-H_{y}^{s}(I, J, K-1)^{n-\frac{1}{2}}}{\Delta z}\left(\frac{\Delta t}{\varepsilon+\sigma \Delta t}\right)
\end{aligned}
$$

where $s$ denotes the scattered field, $i$ denotes the incident field, and $n$ is the current time step. Similar results are obtained for the $y$ and $z$ components of the electric field, and for the magnetic field.

\section{3: Material Parameters}

The above formulation assumes constant material parameters $\varepsilon$ and $\mu$ (for the magnetic version not shown). However, for a realistic conductor, these values are a function of frequency. Thus, in order to accurately represent field responses from a realistic material, a formulation for frequency dependant material parameters must be implemented. For this reason, xFDTD allows the user to specify materials that have frequency dependant permittivity and permeability, following a modified Debye formulism, which is based on the Drude model:

$$
\tilde{\varepsilon}(\omega)=1+\frac{\omega_{p}^{2}}{\omega\left(i v_{c}-\omega\right)},
$$


where $\omega_{p}$ is the plasma frequency, $\omega$ the angular frequency, and $v_{c}$ the collision frequency.

The Drude model is not directly usable because it provides a poor approximation for the index of refraction of noble metals for large frequency ranges. In this modified Debye formulism, a frequency dependent permittivity, $\tilde{\varepsilon}(\omega)$ (and permeability for magnetic materials) is used:

$$
\tilde{\varepsilon}(\omega)=\varepsilon_{\infty}+\frac{\varepsilon_{s}-\varepsilon_{\infty}}{1+i \omega \tau}+\frac{\sigma}{i \omega \varepsilon_{0}},
$$

where $\varepsilon_{\infty}$ is the infinite frequency permittivity, $\varepsilon_{s}$ is the static permittivity, $\tau$ is the relaxation time and $\sigma$ is the conductivity of the dispersive material. Unfortunately, there is no automation for the process of calculating these processes in XFDTD, all material parameters must be calculated by hand and put into the software manually. All of the models done used the parameters set forth by Krug et al, for gold, $\varepsilon_{\mathrm{s}}=-12987, \varepsilon_{\infty}=9.012, \sigma=1.464 \times 10^{7} \mathrm{~S} / \mathrm{m}$, and $\tau=8.017 \times 10^{-15} \mathrm{~s}$, which gives a good coverage of wavelengths for the lasers used for TENOM (700-1000 nm) [Krug, Sánchez and Xie, 2002]. All the modeling done in the following chapters is done at $800 \mathrm{~nm}$ which is reasonably close to the $830 \mathrm{~nm}$ used in the current generation of TENOM systems.

\section{4: Realistic Modeling Spaces - Limitations of FDTD}

As previously mentioned, one of the major hurdles to modeling in general is the ability to use computational power effectively to produce not only realistic results, 
but realistic simulations. It does no good to model the field response of a nano-probe if your system is comprised of micron sized Yee cells. Conversely, the computational power required to model large spaces using small cells can quickly reach unobtainable requirements. Using a rough estimate, every cell will require 30 bytes of memory to be computed. This means that for a $100 \times 100 \times 100$ cell model space, roughly $30 \mathrm{Mb}$ of RAM is required. This also means that with $30 \mathrm{Mb}$ of RAM, and $1 \mathrm{~nm}$ cell sizes, it is only possible to model a space that is roughly $.1 \mu \mathrm{m}$ in each dimension. For a space that is 1 meter across, using $1 \mathrm{~nm}$ cells, the RAM requirement is approximately $30 \mathrm{~Gb}$.

One useful feature of xFDTD is sub-meshing, or adaptive meshing. This allows for finer detail features to be examined in a space that is comparatively large and is especially useful (as in this case) when examining a feature where fine detail is required but elsewhere is unnecessary. The basic principle behind sub-meshing is that the user can specify a region within the model space that has a different Yee cell size. Thus, it is possible to propagate a wave through free space with large cell sizes and yet examine a very small object, with small cell sizes. The concept of submeshing is illustrated through Figures 2.3-2.6. In Figure 2.3, a $100 \mathrm{~nm}$ radius sphere is approximated in a $\sim 1 \mu \mathrm{m}$ modeling space, using $80 \mathrm{~nm}$ cells. As illustrated by this figure, approximating a sphere with cubes with similar spatial dimensions is not a good approximation and will result in poor field approximations for a sphere. However, to run a model this size would require only $\sim 10 \mathrm{Mb}$ of RAM. The time requirement would also be very low. 


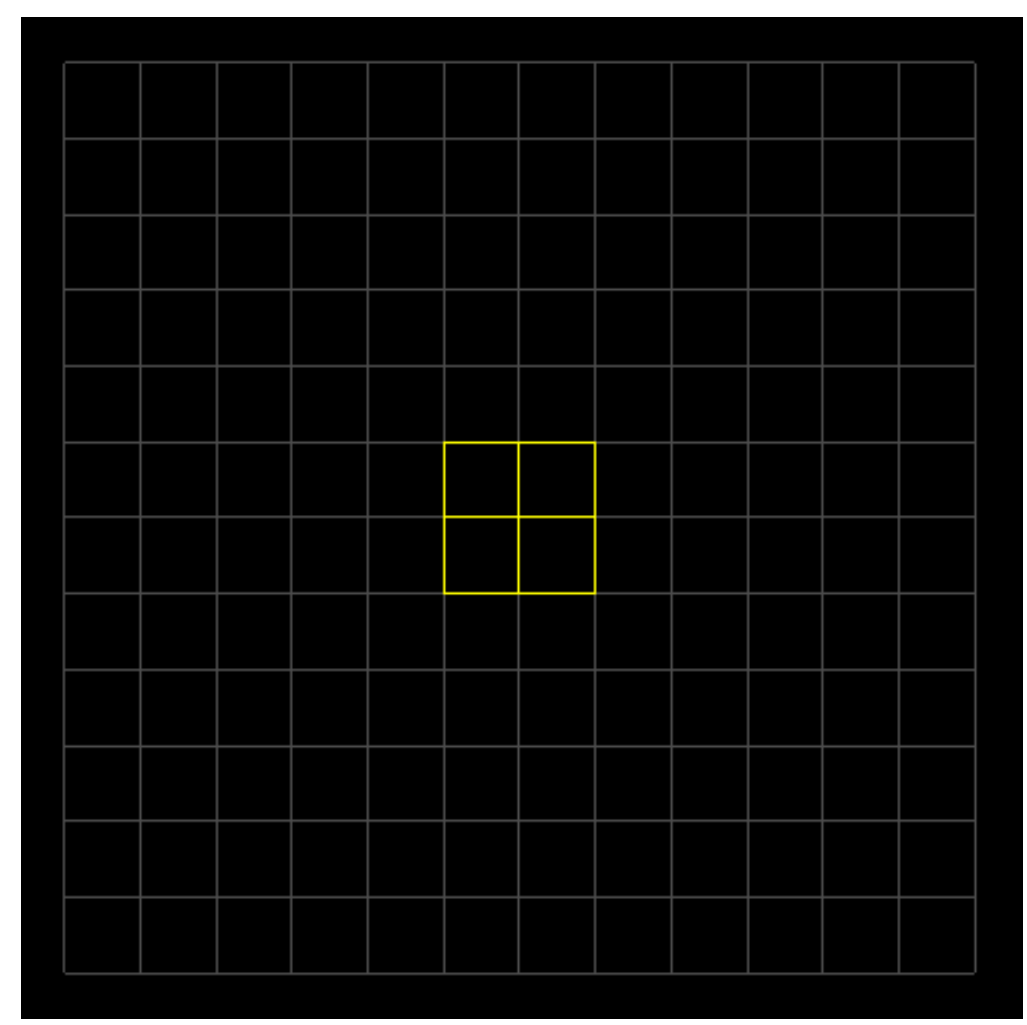

Figure 2.3: $100 \mathrm{~nm}$ radius sphere approximated by $80 \mathrm{~nm}$ cubes.

In the other extreme, a $\sim 1$ micron space with no submeshing and $1 \mathrm{~nm}$ cells approximates the sphere very well. However, to actually run the model is beyond the abilities of available resources and XFDTD is unable to even mesh this configuration due to the resource requirement. It would take around $26 \mathrm{~Gb}$ of RAM to run this model and a very long time, even with a state of the art desktop pc. Figure 2.4 illustrates submeshing of an $80 \mathrm{~nm}$ cell size with a $1 \mathrm{~nm}$ cell submesh. This compromise both represents a reasonable resource requirement and a reasonable approximation to the sphere. The RAM requirement for this configuration is roughly $504 \mathrm{Mb}$, which is reasonable for most desktop pc systems. 


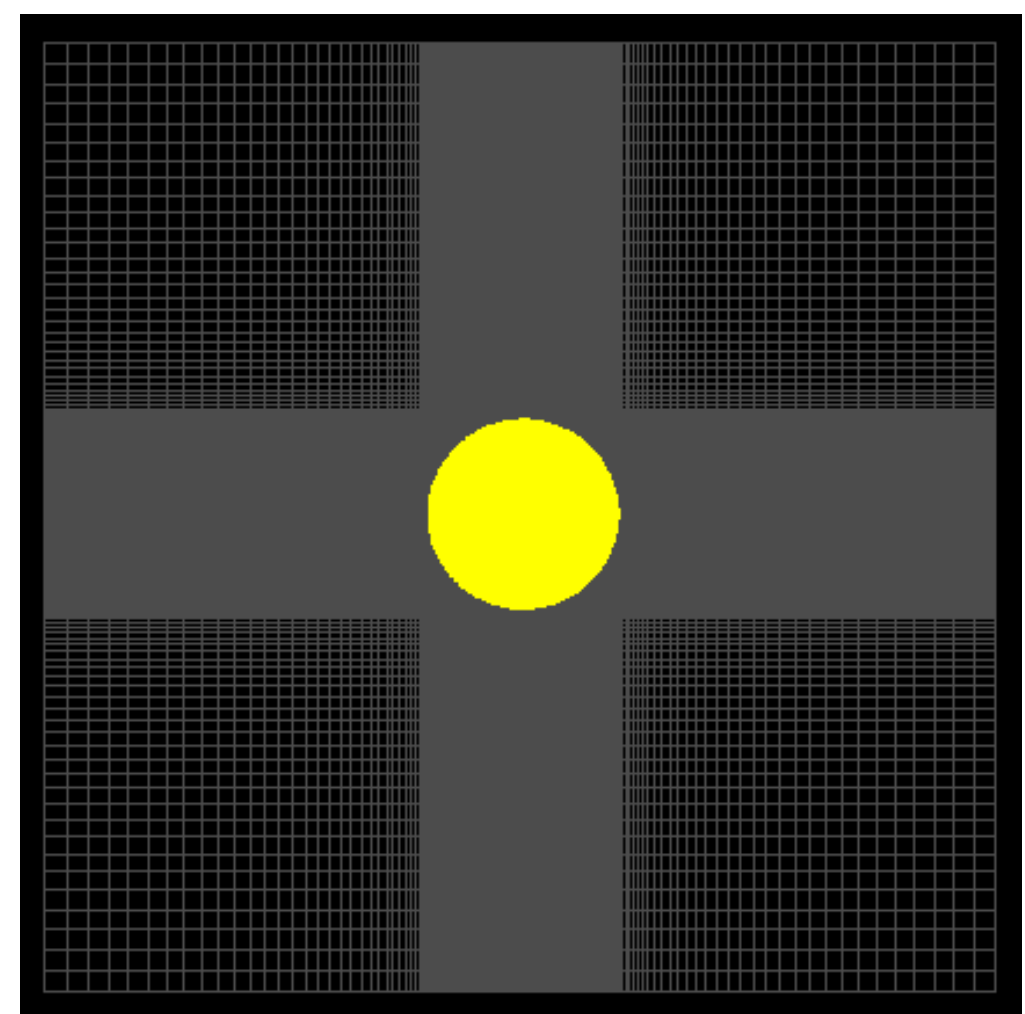

Figure 2.4: An image of the GUi in XFDTD for an $80 \mathrm{~nm}$ cell size with

$1 \mathrm{~nm}$ submeshing for the sphere.

Zooming in on Figure 2.4 gives Figure 2.5, which is the $1 \mathrm{~nm}$ submeshed sphere in an $80 \mathrm{~nm}$ cell base cell space. As shown in this figure, a $1 \mathrm{~nm}$ cell size represents the sphere quite well and as mentioned above, the resource requirements are insubstantial, allowing more complex models to be run without the need of a supercomputer.

Figures 2.6 and 2.7 show the way xFDTD handles transitions from normal meshes to submeshes. In 2.6, only quadrant 4 is submeshed to $1 \mathrm{~nm}$. xFDTD basically submeshes a strip along the entire space in the plane that is selected. For this scenario, if the sphere is at the origin, the submeshing is from 0 to $100 \mathrm{~nm}$ in each axis. This results in a strip from -.5 microns to .5 microns in each axis that is 
submeshed at $1 \mathrm{~nm}$. In other words, the points $(-.5,0),(-.5, .1),(.5,0)$ and $(.5, .1)$ form a rectangle in which every cell is submeshed to $1 \mathrm{~nm}$ in the y direction. Similarly, the points $(0,-.5),(.1,-.5),(0, .5)$ and $(.1, .5)$ describe the region of $1 \mathrm{~nm}$ submeshing in the $\mathrm{x}$ direction. Quadrant 4 is where the 2 (or 3 including the $\mathrm{z}$ axis) submeshed regions combine to form the completely submeshed region. Quadrant 1 is a transition region only, there is no submeshing specifically set, however this region is still affected as seen in Figure 2.7. As seen in this figure, it takes roughly 5 microns to transition from $1 \mathrm{~nm}$ to $80 \mathrm{~nm}$ cells. This means that any features near the submeshed region will be at least partially submeshed. This is done to eliminate artifacts and make the modeling space continuous.

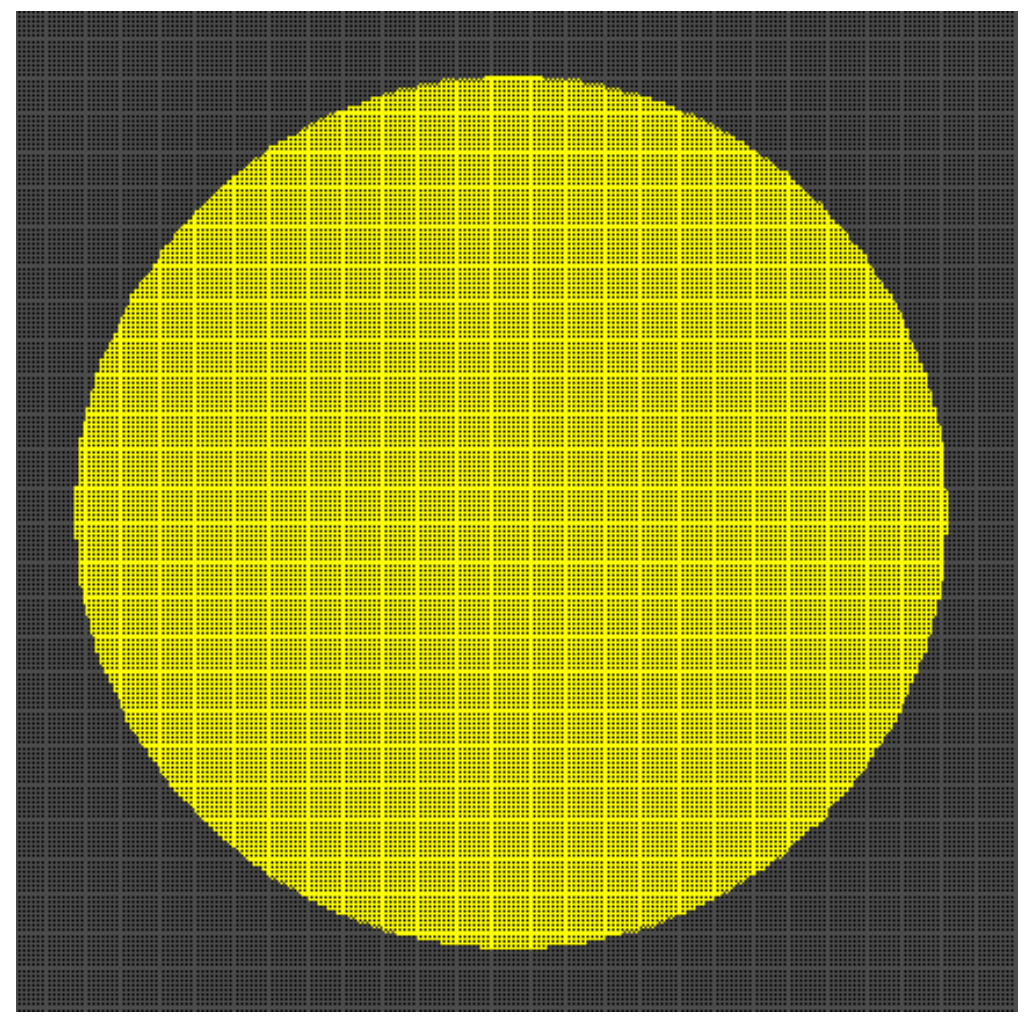

Figure 2.5: zoomed in on the submeshed region from Figure 2.4. 


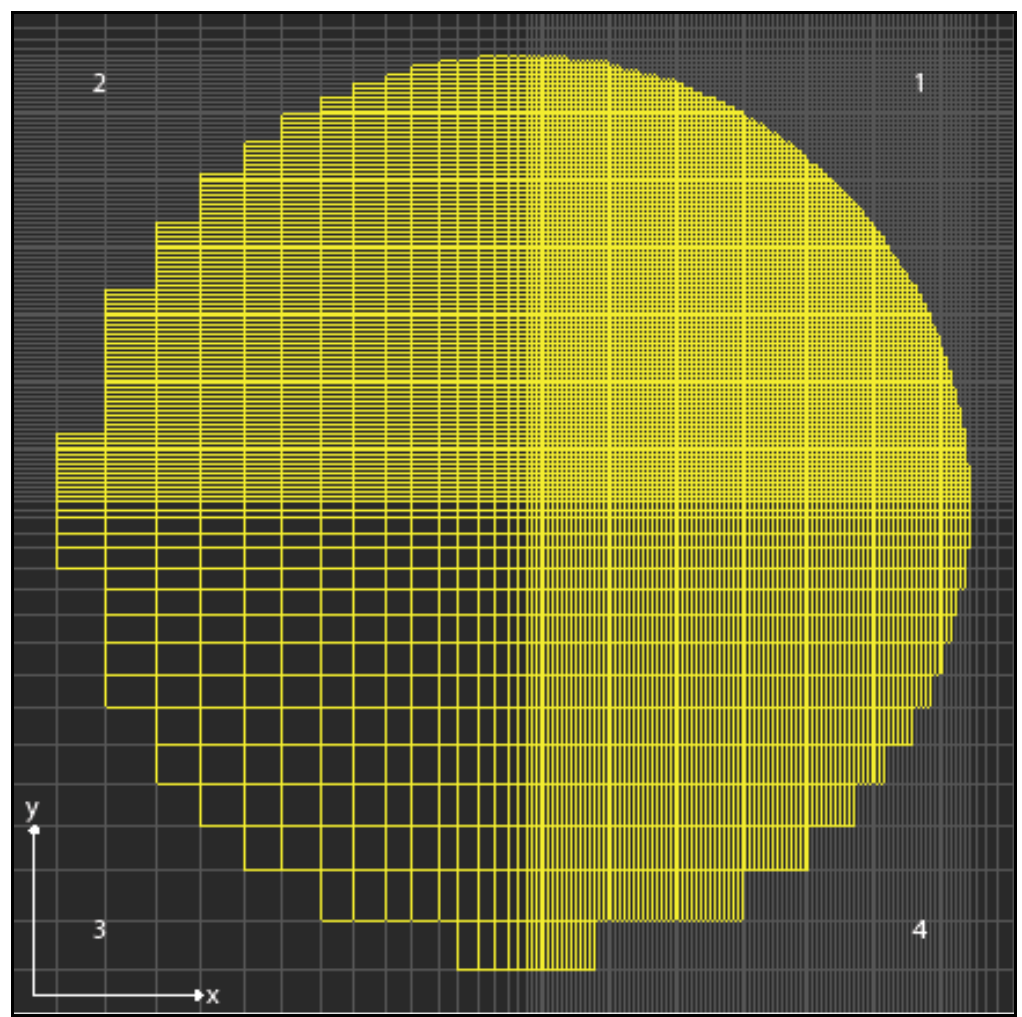

Figure 2.6: partial submeshing of a sphere.

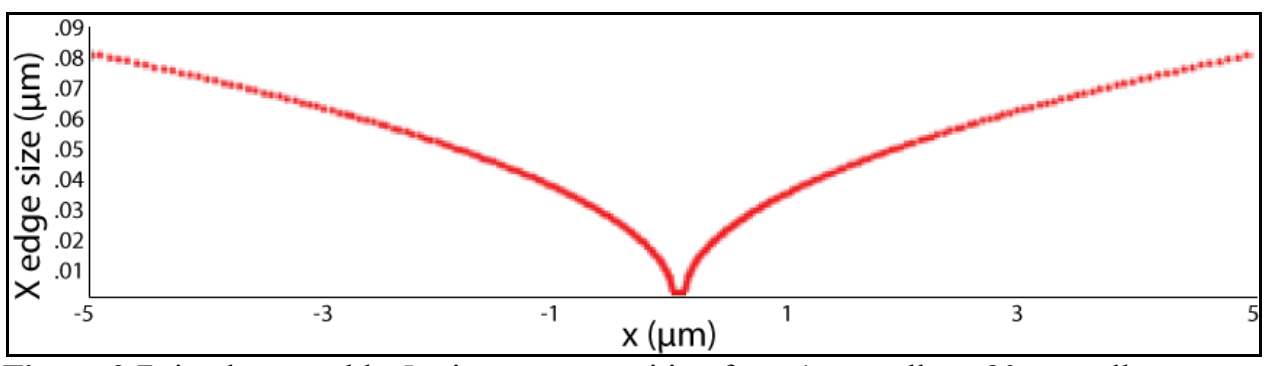

Figure 2.7: it takes roughly 5 microns to transition from $1 \mathrm{~nm}$ cells to $80 \mathrm{~nm}$ cells.

The largest benefit gained from the submeshing is the ability to model a lens.

Without submeshing, this would not be possible utilizing the available resources.

Nowhere else in the literature is the lens modeled in systems such as this, because it is 
so resource intensive. With submeshing, it was possible to model the lens/tip system with Pentium 4 desktops with only $2 \mathrm{~Gb}$ of RAM. This allowed a focused point to interact with the probe, rather than a mere plane wave, giving more accurate results. 


\section{Chapter 3: Modeling of Probes}

\section{1: Varying location of focal volume}

The main point of this section is to show that by varying the location of the focal volume and polarization of light, the effect of polarization can be examined, and shown to be unimportant in the symmetrical probe geometry, which is not the case in the asymmetric geometry. It will also be shown that focusing in the bulk region, at points $\mathrm{A}$ or $\mathrm{C}$ is necessary for background suppression. Figure 3.1 outlines the positions where the focal volume was placed and the corresponding FDTD field snapshots follow. In this figure and in the modeling runs done, the distance between the tip and the lens system is $9 \mathrm{~nm}$. This is also an accurate representation of the current TENOM system.

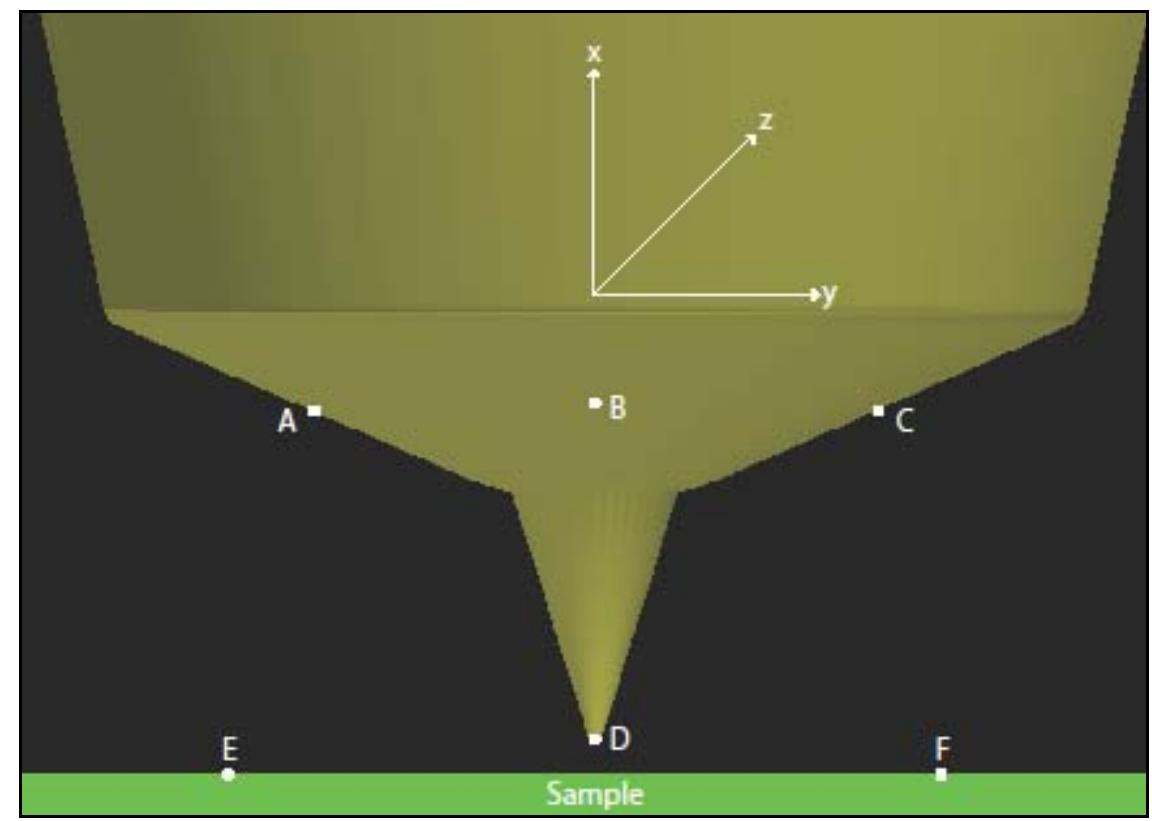

Figure 3.1: The focal volume placement in the xy-plane.

Due to symmetry, only points A, B, D, and E are shown below. Point F will be the same as $\mathrm{E}$, and point $\mathrm{C}$ will be the same as $\mathrm{A}$. One figure of point $\mathrm{C}$ will be 
presented to illustrate this; otherwise the field snapshots of $\mathrm{C}$ and $\mathrm{F}$ are discarded as extraneous.

For each location, a half wavelength is shown in $1 / 8$ wavelength steps. Since it is a sine wave that is propagating through the material and only magnitude is considered, the negative portion of the sine wave excitation is the same as the positive portion. This is illustrated by comparing Figure 3.5 to Figure 3.7, and Figure 3.6 to Figure 3.8. In order to truly approximate $1 / 8$ wavelength steps, time steps of $\sim 63$ would have to be taken, but since only intervals of 5 were available, time steps of 65 were taken. Therefore, there are slight differences in the 2 half wavelengths, because data was taken in intervals of 5 time steps during the modeling calculations. However, it is safe to approximate the 2 halves of a wavelength as equal and therefore only the first half wavelength will be presented for all images subsequent.

The source for all the modeling is an $800 \mathrm{~nm}(\sim 374,628 \mathrm{GHz}$ in air $)$ sinusoid propagating in the $\mathrm{x}$ direction, focused to a "point" by a glass lens as shown in Figure 3.2. Figure 3.2 is not to scale with the rest of the images in this chapter, in terms of field intensity. The half angle is roughly $50^{\circ}$ which gives an NA of about 1.18 for glass with an index of refraction of 1.541. Each model is allowed to run for 20,000 time steps and data is taken from 15,000 to 20,000 time steps in increments of 5, for a total of 1,000 data points. The number of time steps is dictated by the condition that the incident field reaches a steady state, which will occur after roughly 10 wavelengths have propagated.

The workspace consists of $80 \mathrm{~nm}$ cells, with the tip apex submeshed down to 3 $\mathrm{nm}$. This is within a space that is roughly $49,367,500$ cells, depending on the specific 
model (490x325x310 as one example). This usually takes up around $10 \mathrm{~Gb}$ of hard drive space, which can be problematic, depending on the resources available. It may be desirable to save only the last 1,000 time steps $(19,000$ to 20,000$)$ in order to save disk space.

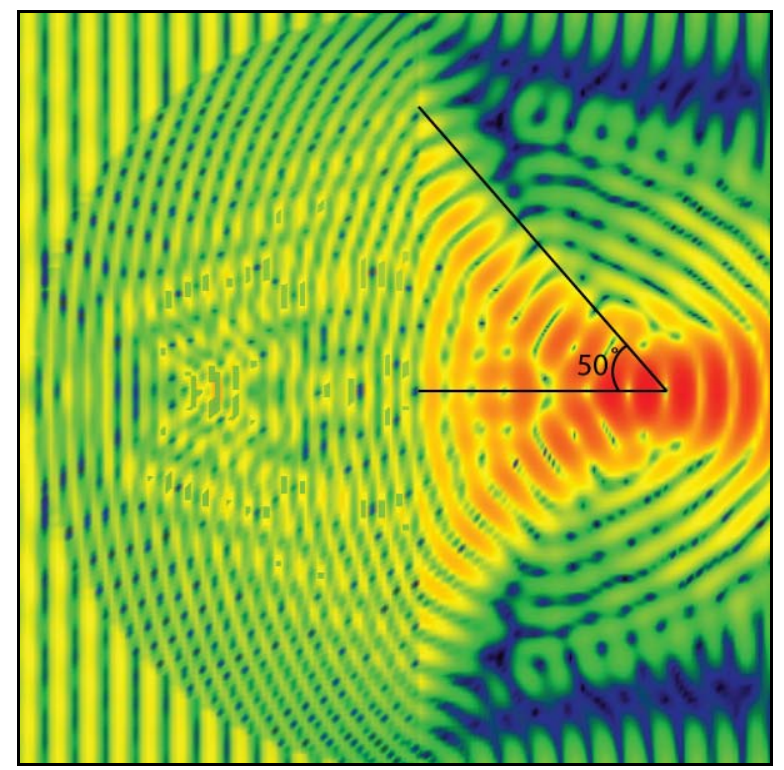

Figure 3.2: Lens with an index of refraction equal to 1.541. A NA of 1.18 is calculated.

Figure 3.3 represents the scale used for all the following images, making comparable the field intensity for all images. To find the field enhancement at any point, squaring the field intensity is needed. Therefore, for a $70 \mathrm{~V} / \mathrm{m}$ intensity, the field enhancement is $4,900 \mathrm{~V}^{2} / \mathrm{m}^{2}$, which is slightly higher than the actual maximum field enhancement found in any snapshots. This was done to keep red as the maximum for all snapshots and ensuring that $70 \mathrm{~V} / \mathrm{m}$ was the upper limit to the field found in all models.

Figure 3.3: The scale used in the following figures. 
The key to interpreting these images is that a field will excite the sample and it is desirable to only excite the sample in the region which is sub-diffraction limited. This is accomplished by a high field at the apex of the tip, with a low field everywhere else. This gives a high signal to noise ratio, which is very important in near-field imaging. Background suppression has traditionally been a problem and is currently a focus of much energy within the community. It is undesirable to have a field anywhere but at the apex of the tip because the desired effect is sample excitation within a sub-diffraction limited spot.

The first of the snapshots are shown in Figures $3.4-3.7$ and represent the field response in the asymmetric probe design at focal spot $\mathrm{C}$ in Figure 3.1. This is for comparison to snapshots of the symmetric design, shown later in the chapter. This asymmetric design has been used to acquire images in the current TENOM system and works very well for both topography and near-field. Figures 3.4 and 3.5 show the field response with the polarization in the $\mathrm{y}$ direction, with $\mathrm{x}$ the propagation direction. These snapshots show why the asymmetric probe has been a good design to date; there is a high field at the apex and for the most part, a low field everywhere else, at least comparatively. The axes shown in Figures 3.4 and 3.5 are used for their corresponding snapshots for the remainder of the chapter.

However, when the polarization is rotated into the $\mathrm{z}$ direction, this design becomes useless for near-field imaging, as shown in Figures 3.6 and 3.7. These snapshots show that the apex field is far lower than the background and therefore only the background, diffraction limited far-field will excite the sample, producing only noise. This shows that the asymmetric probe design is very much dependant on 
polarization and in the physical system, it is necessary to rotate polarization once focused on the probe until a high signal count is achieved.

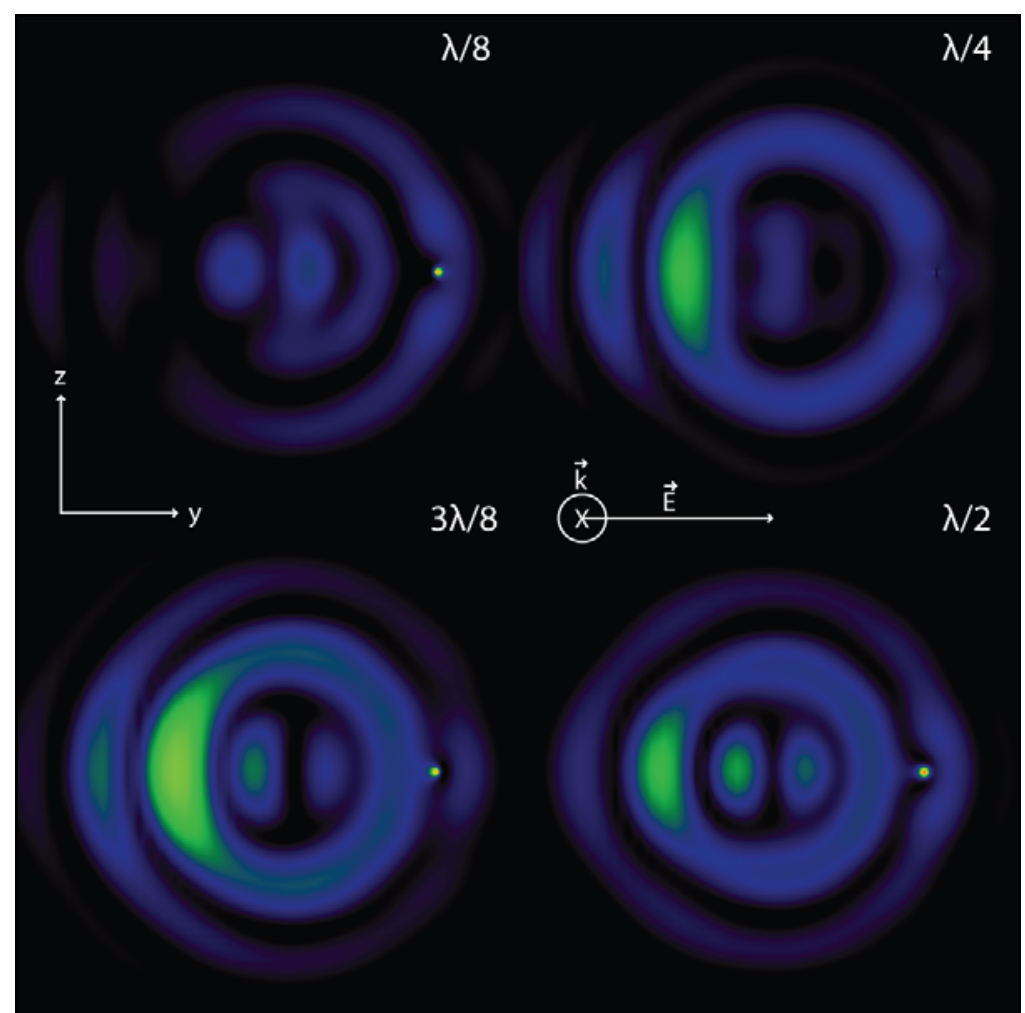

Figure 3.4: The yz-plane field response for an asymmetric probe, with polarization in the y direction. 


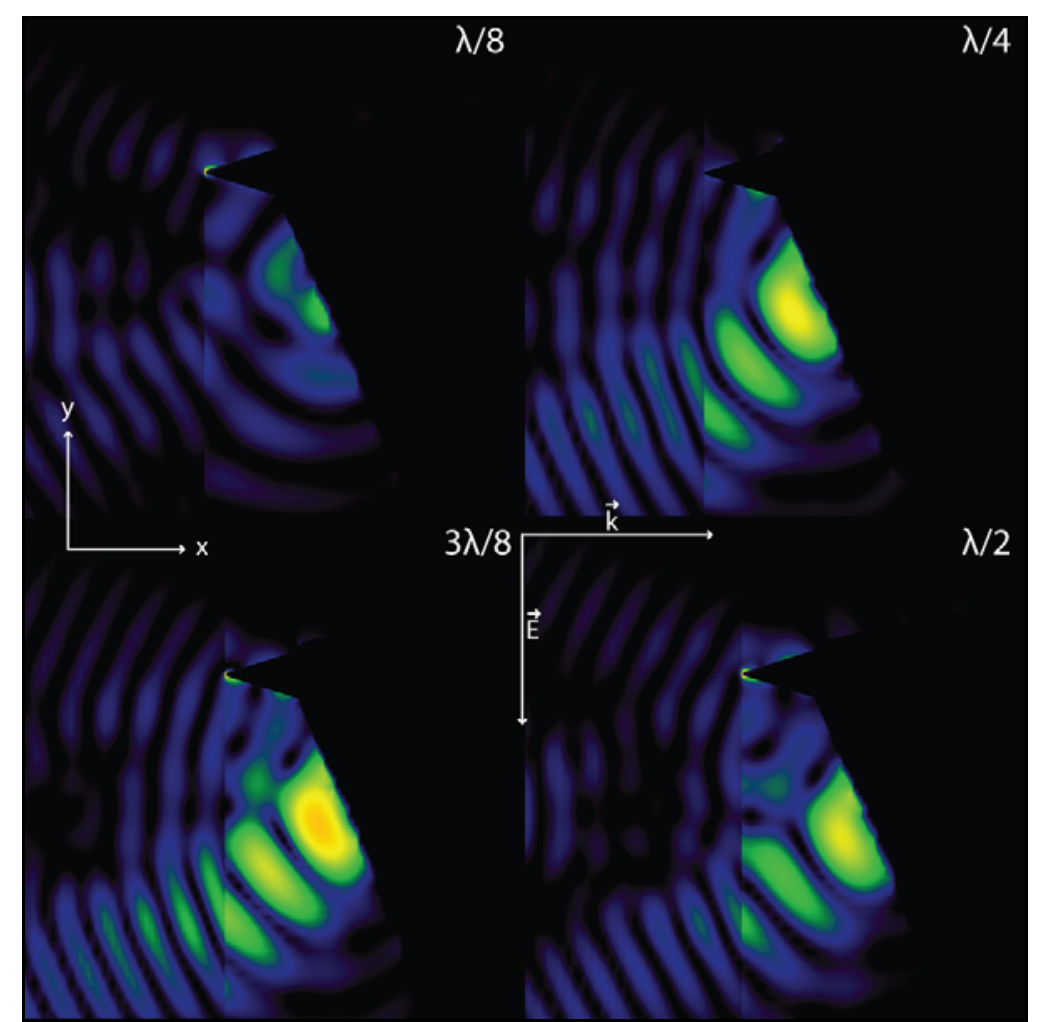

Figure 3.5: The xy-plane field response for an asymmetric probe with polarization in the y direction.

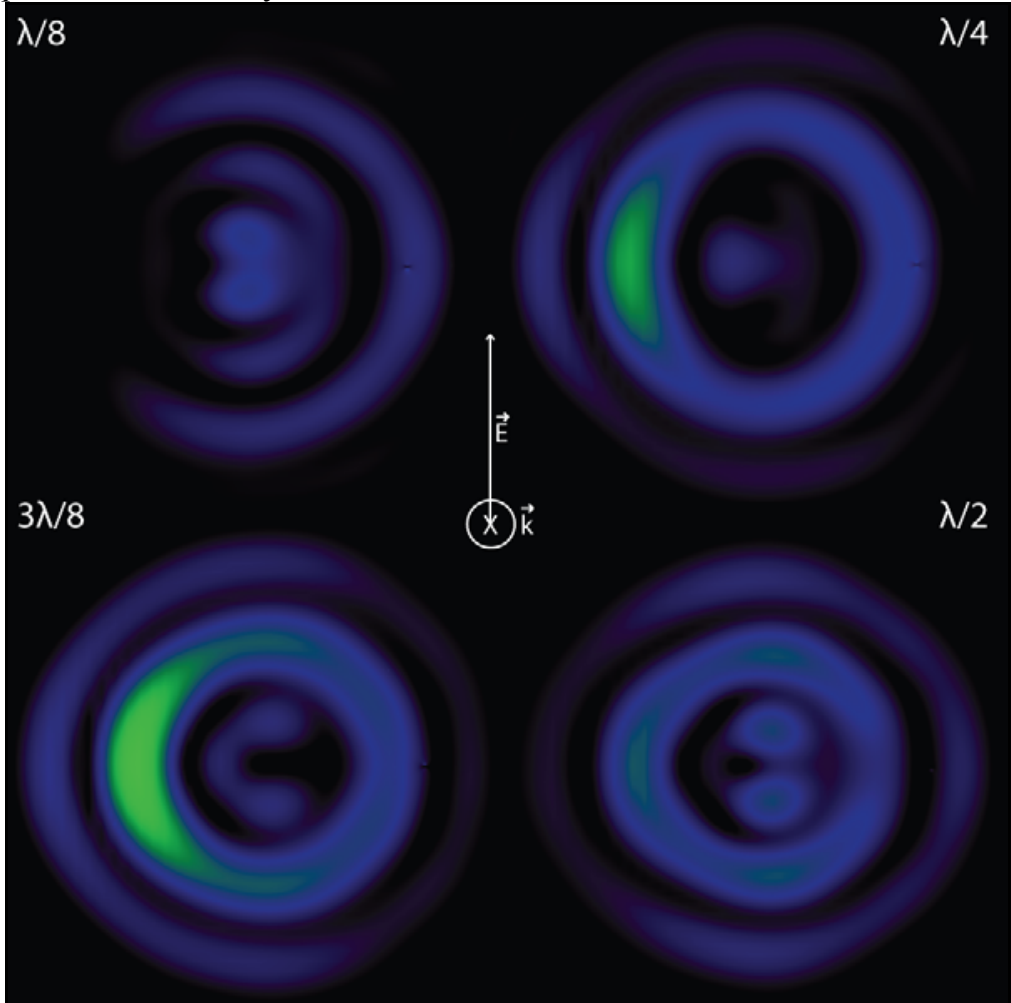

Figure 3.6: The yz-plane field response for an asymmetric probe, with polarization in the $\mathrm{z}$ direction. 


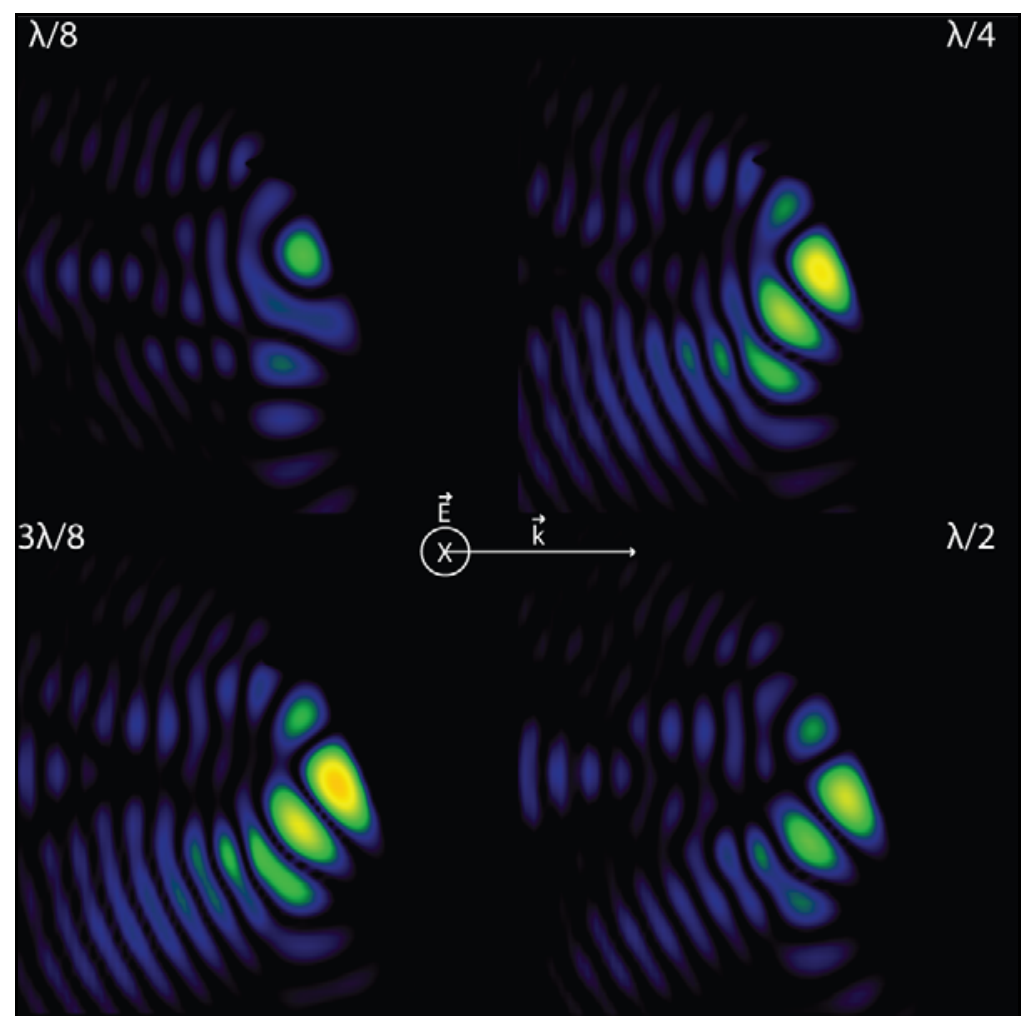

Figure 3.7: The xy-plane field response for an asymmetric probe, with polarization in the $\mathrm{z}$ direction.

By propagating the incident field in the $\mathrm{x}$ axis with a polarization in the $\mathrm{y}$ direction; it is shown that in Figures 3.9 and 3.10, corresponding to point A in Figure 3.1, a very good signal to noise ratio is predicted. The tip apex field is clearly visible and has the highest field of all points, at all 4 time steps. This is the ideal imaging scenario for this geometry; the small end radius will provide for good topography, while the high field at that small tip will provide good near field imaging with a low background. In this snapshot the beam is focused away from the actual imaging center, which reduces the background signal, by removing the far-field component from the portion of the sample being imaged. With the polarization of the field driving charge from the bulk to the tip apex, it is repeatedly shown that the highest field enhancements result. This is really the key; driving charge from the bulk to the tip apex and is why polarizations 
in the wrong direction produce poor imaging conditions. This is also why a very sharp tip alone will not produce desirable results which can be seen in Figure 3.8. There is no bulk from which a large quantity of free electrons can be driven to a sharp point creating a large charge density, so the charges oscillate between the sides of the sharp tip and never make their way down to the apex.

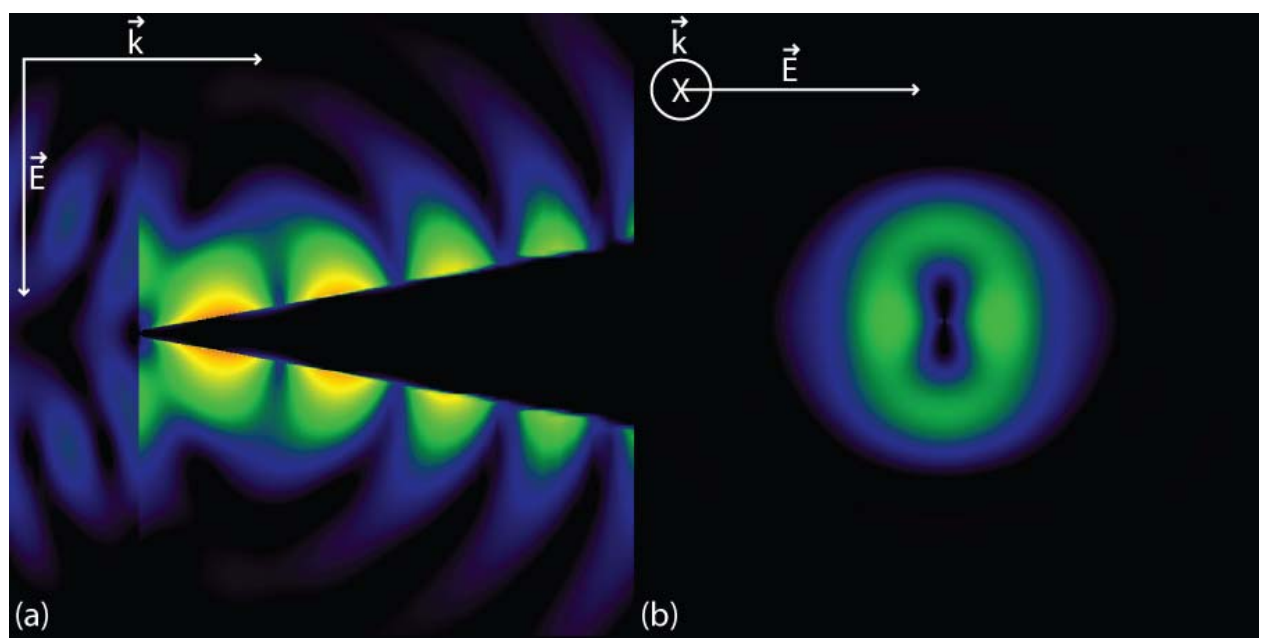

Figure 3.8: A sharp tip and its field response. 


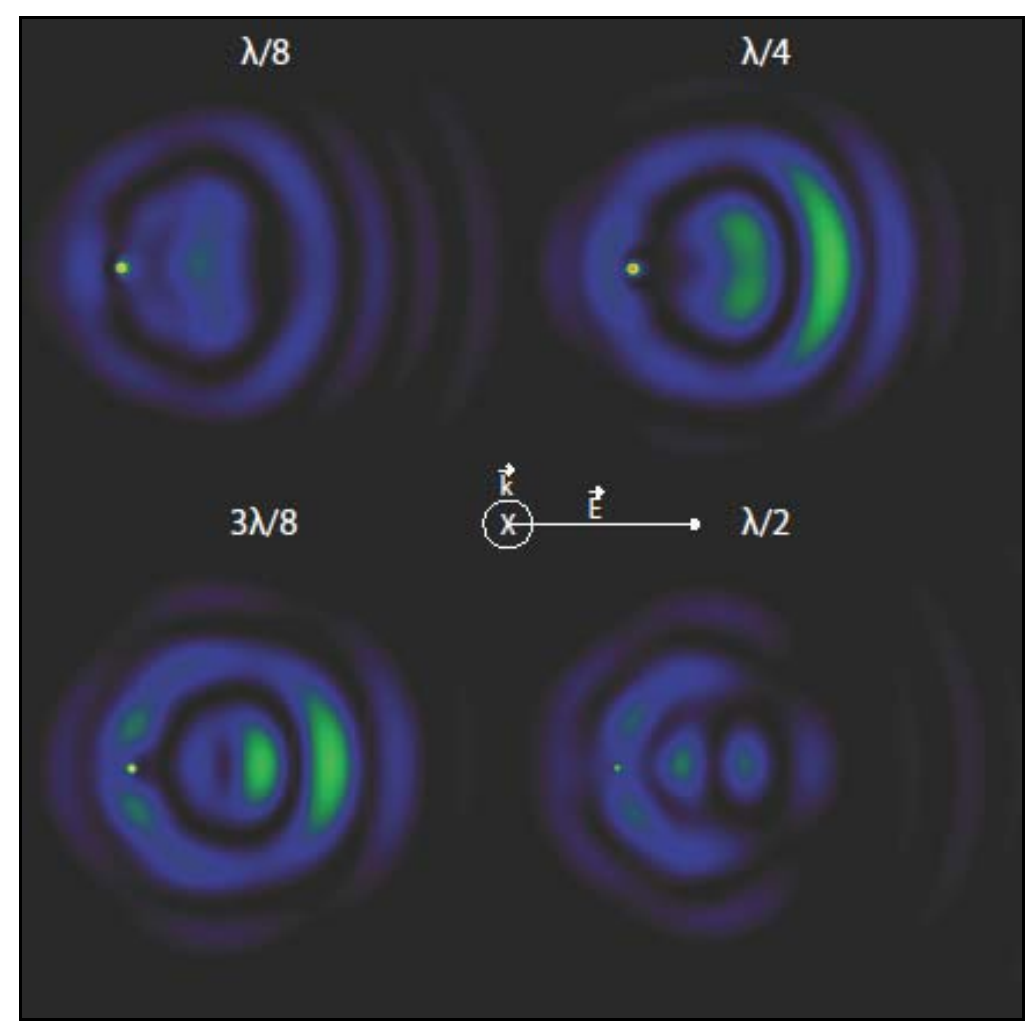

Figure 3.9: The yz-plane field response for a y polarization at point A in Figure 3.1.

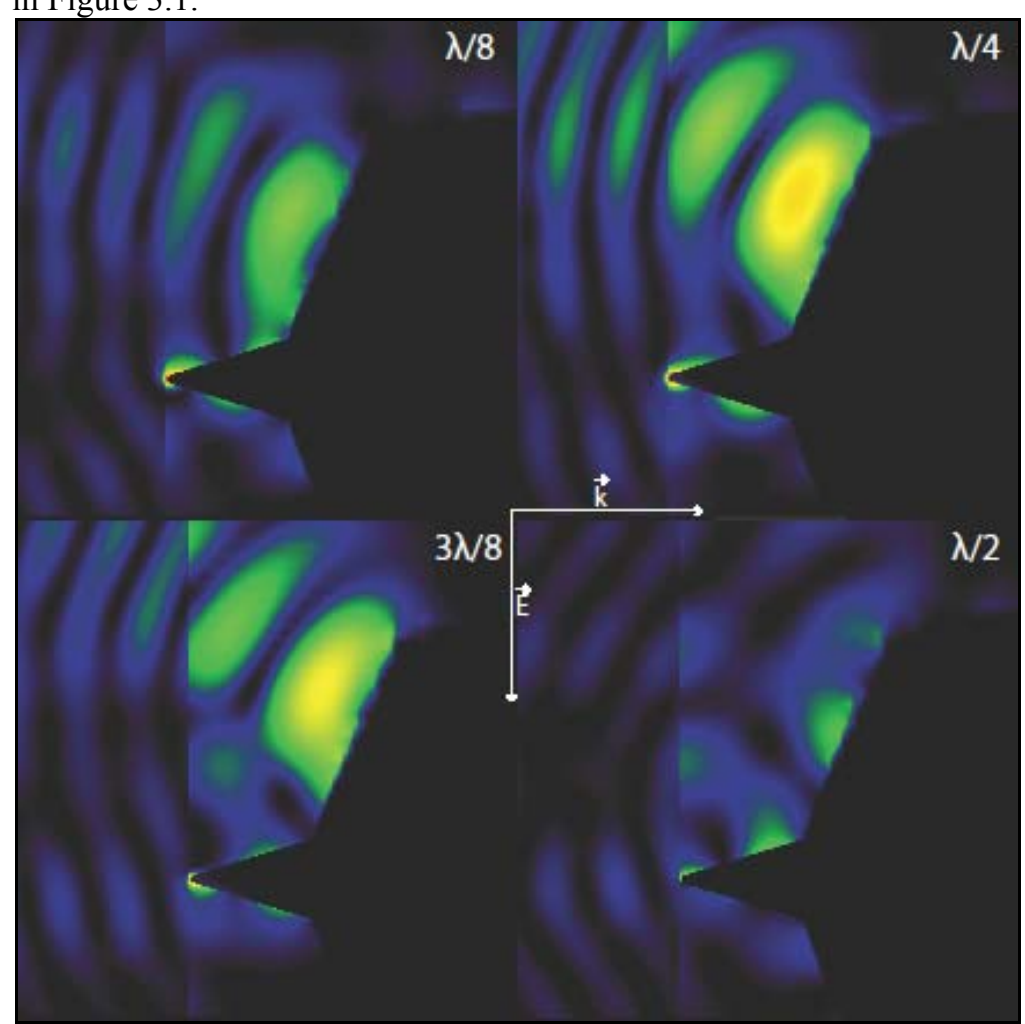

Figure 3.10: The xy-plane field response for a y polarization at point $\mathrm{A}$ in Figure 3.1. 


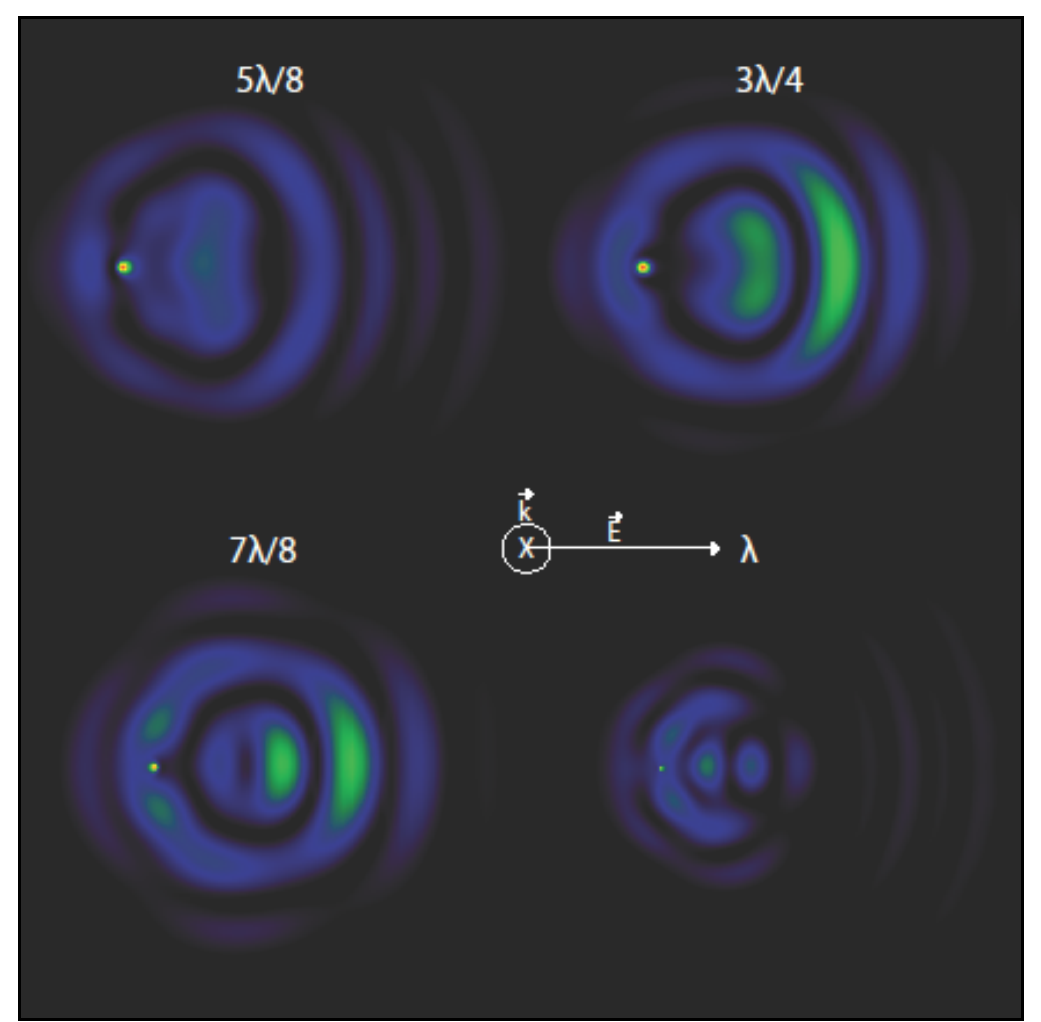

Figure 3.11: The yz-plane field response for the second half of the wavelength at point $A$ in Figure 3.1. The polarization is in $\mathrm{y}$.

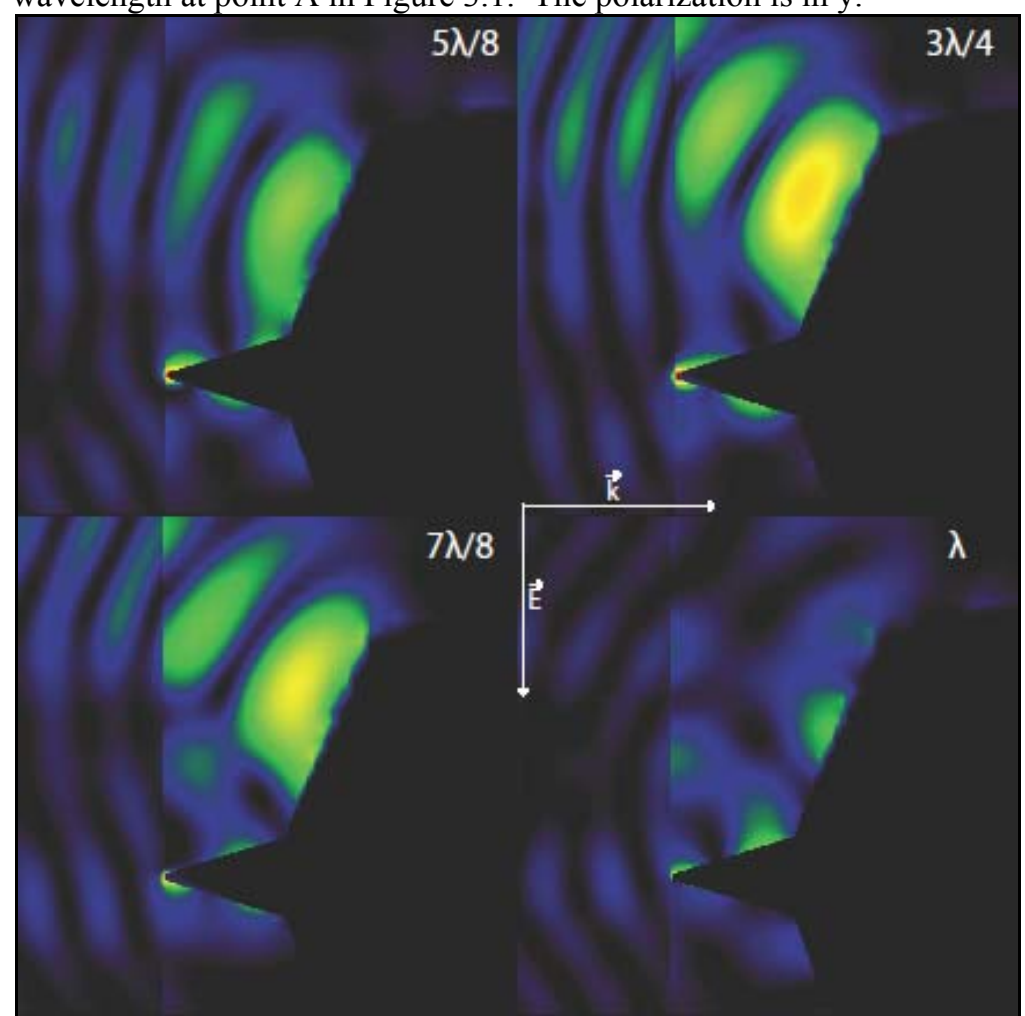

Figure 3.12: The xy-plane field response for the second half of the wavelength at point A in Figure 3.1. The polarization is in y. 


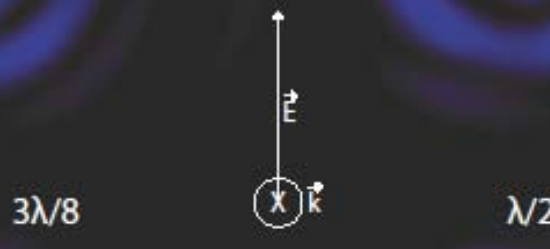

Figure 3.13: The yz-plane field response for a $\mathrm{z}$ polarization at point $\mathrm{A}$ in Figure 3.1.

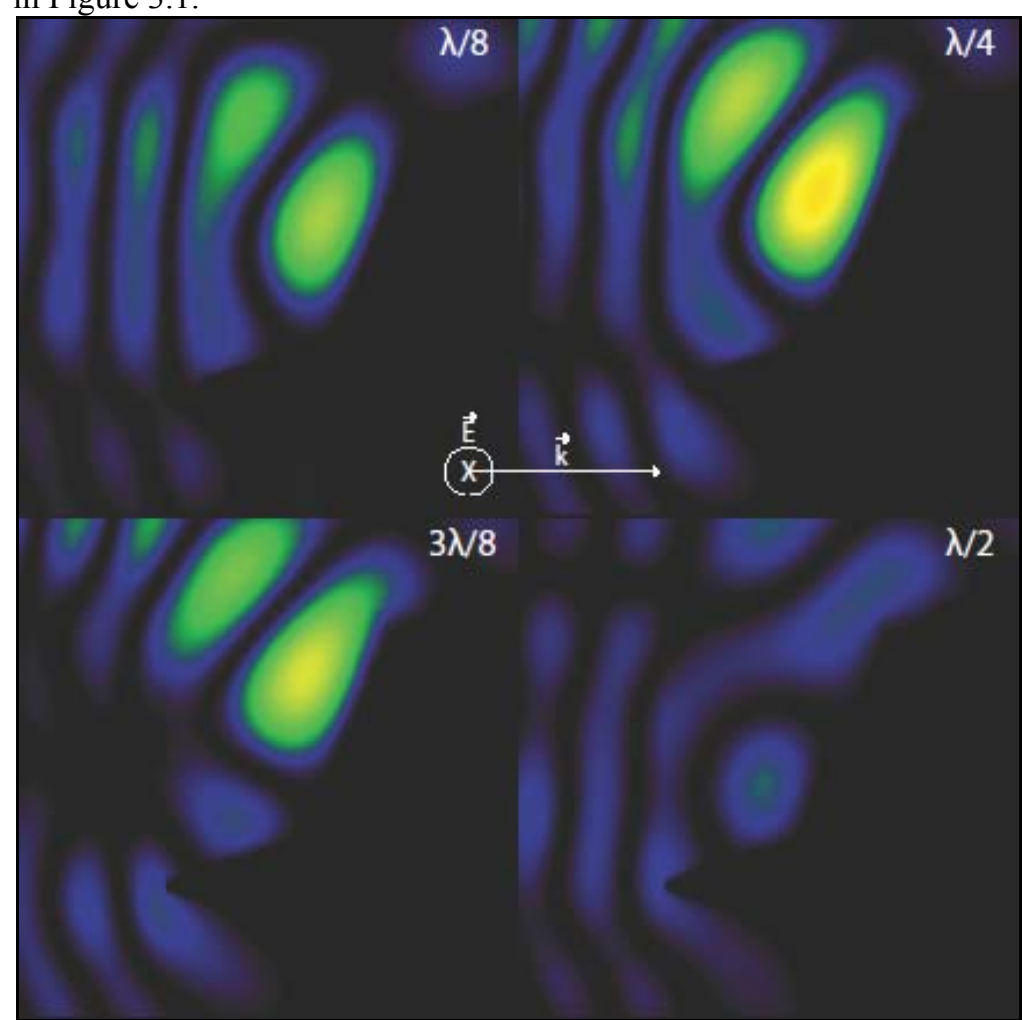

Figure 3.14: The xy-plane field response for a $\mathrm{z}$ polarization at point $\mathrm{A}$ in Figure 3.1. 
As mentioned above, switching the polarization at focal point A, from $\mathrm{y}$ to the $\mathrm{z}$ direction leads to very poor field enhancement. This can be seen in Figures 3.13 and 3.14. Nowhere in these figures is the tip apex field greater than that of the background. This is due to the direction of charge driving in the direction perpendicular to the tip apex. These are very comparable to the asymmetric design. However, the symmetric design allows for some flexibility in utilizing many possible polarizations, as explained later, whereas the asymmetric probe can only utilize a single polarization direction for ideal imaging conditions.

Moving the focus to point B in Figure 3.1, it is seen through Figures 3.15 and 3.16 that a low signal to noise ratio would result, due to the high background field. This is with the polarization in the y direction. However, as can be seen in Figures 3.17 and 3.18 with a $\mathrm{z}$ polarization, the polarization direction doesn't matter at this location; poor near-field imaging will result no matter what the polarization. By rotating the polarization by $90^{\circ}$, a similar rotation of the field response is seen. This is very apparent by comparing the $\lambda / 2$ pane in Figures 3.15 and 3.17. The lobes in Figure 3.15 (with a y polarization) are along the y axis but when the polarization is in the $\mathrm{z}$ axis, the lobes are along the $\mathrm{z}$ axis. This is with the focus within the tip apex/sample plane, which means that the focused light from the lens is passing through the sample at the imaging location. This is opposed to points $\mathrm{A}$ and $\mathrm{C}$, where the focus is away from the imaging location. Hence, at focal points $\mathrm{A}$ and $\mathrm{C}$, a comparatively low background is predicted, whereas the far-field contribution at B and $\mathrm{D}$ is high. 


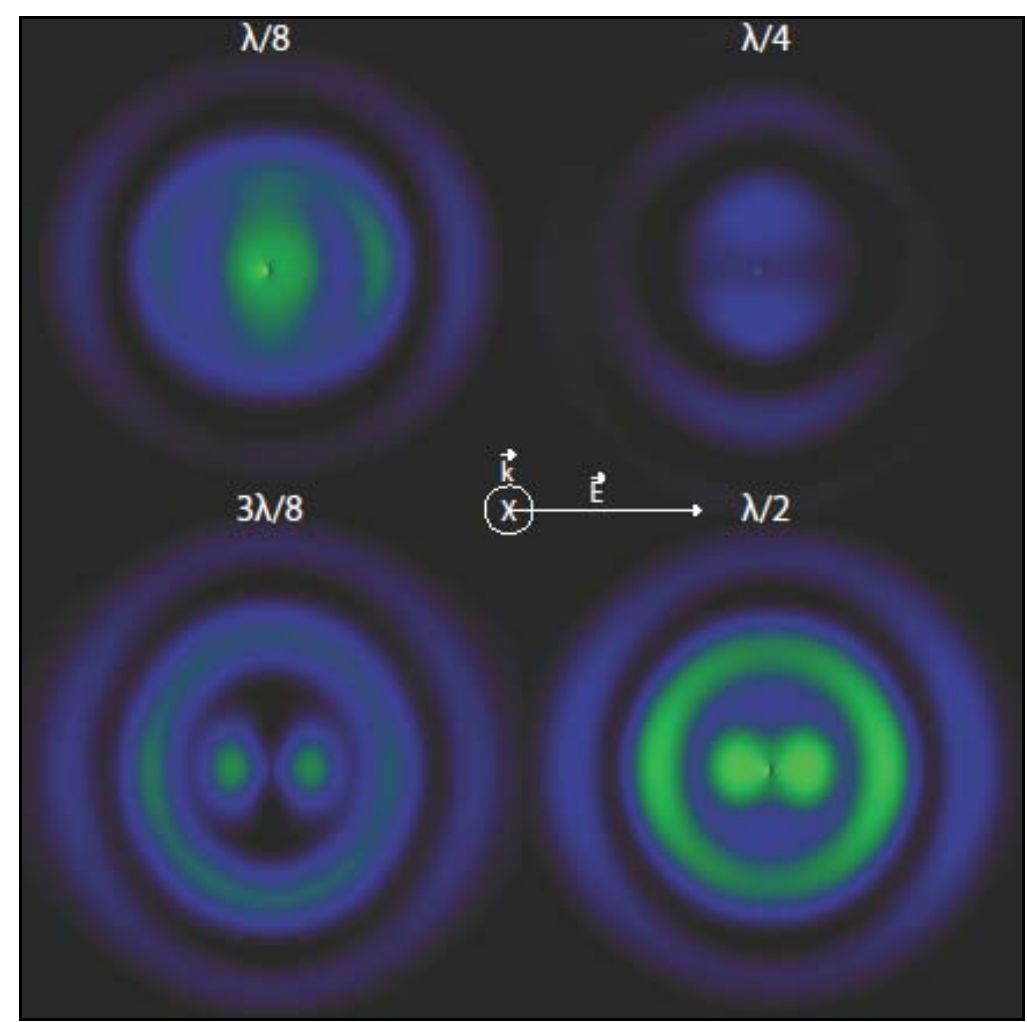

Figure 3.15: The yz-plane field response for a y polarization at point B in Figure 3.1.

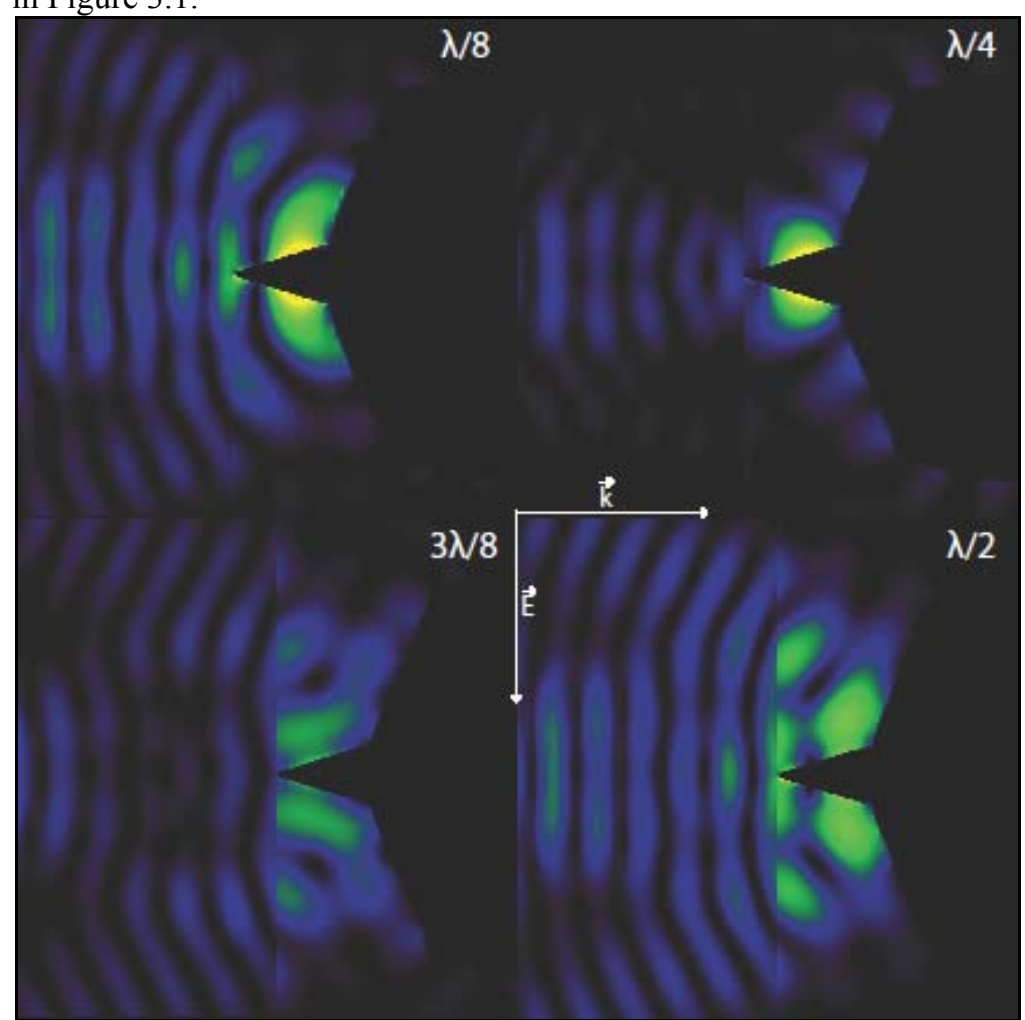

Figure 3.16: The xy-plane field response for a y polarization at point $\mathrm{B}$ in Figure 3.1. 


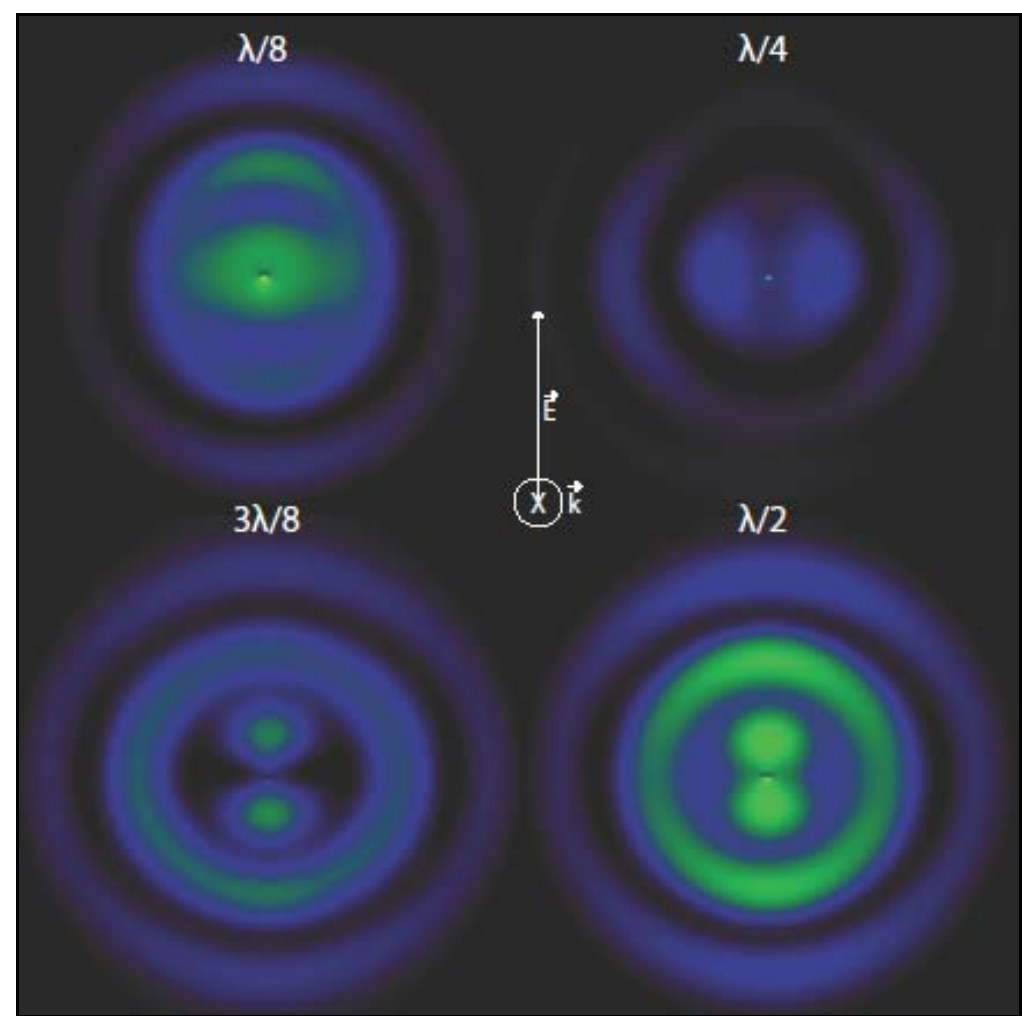

Figure 3.17: The yz-plane field response for a y polarization at point $\mathrm{B}$ in Figure 3.1.

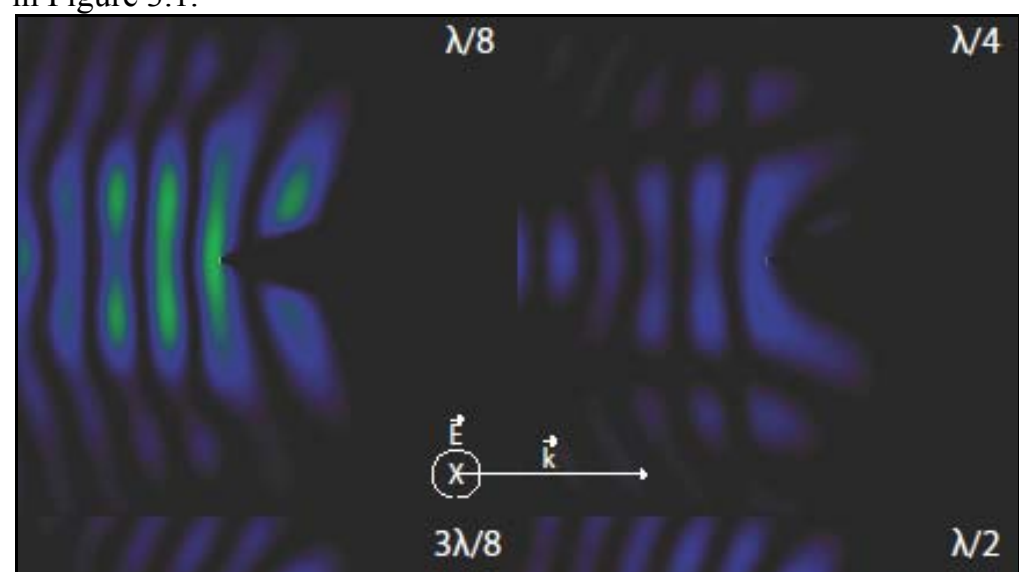

Figure 3.18: The xy-plane field response for a $\mathrm{z}$ polarization at point $\mathrm{B}$ in Figure 3.1. 
Figure 3.19 shows the field response for point C. This shows the symmetrical response within the geometry and allows for the discarding of points $\mathrm{C}$ and $\mathrm{F}$. In comparison to focal point $\mathrm{A}$ in Figure 4.5, point $\mathrm{C}$ is the mirror image, which demonstrates the polarization dependence removal of the asymmetric tip design. This is exactly what should happen; the focus is merely moved from one side of the tip apex to the other, focused within the bulk region, with the polarization remaining the same. They should give the exact same field response due to symmetry and as the figures show, they do.

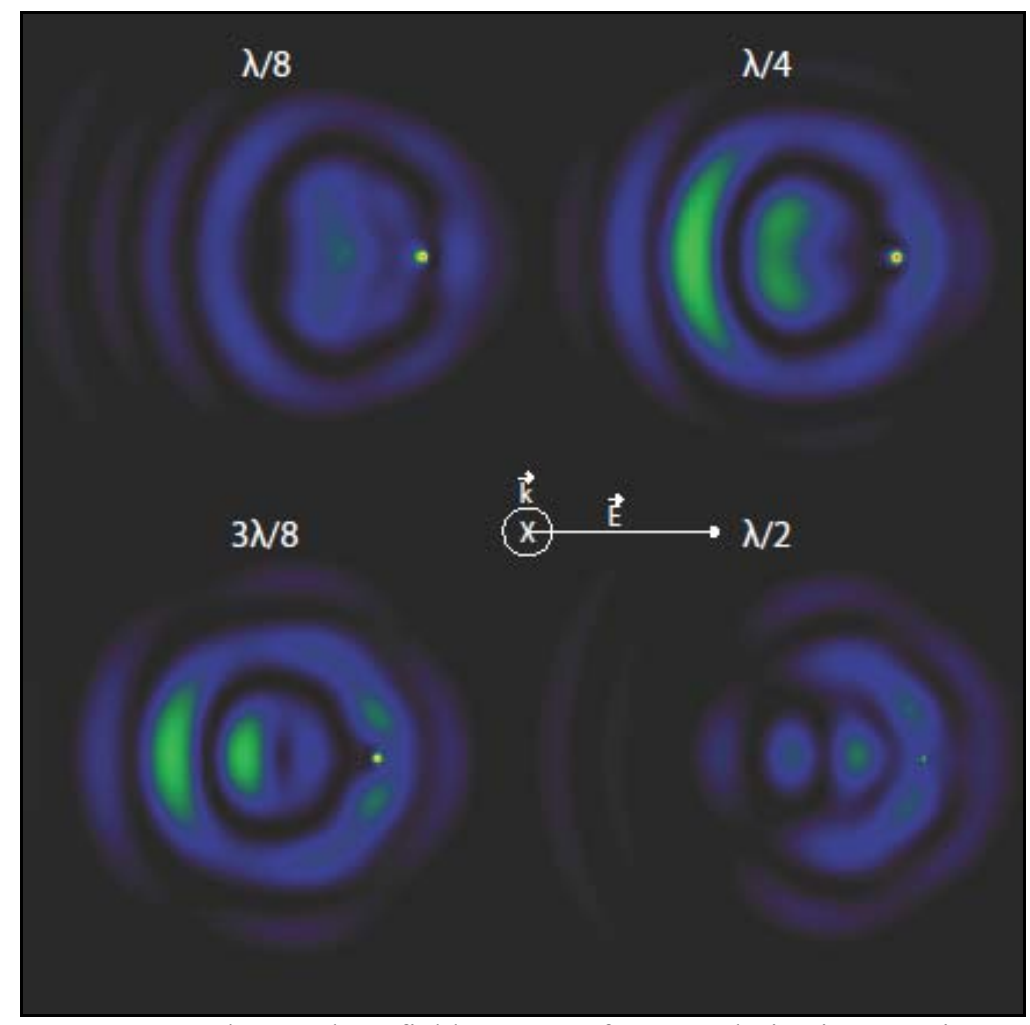

Figure 3.19: The yz-plane field response for a y polarization at point $\mathrm{C}$ in Figure 3.1.

However, to use the phrase "polarization dependence removal" is a slight misnomer, because in actuality there is a necessary polarization associated with each focal position in the yz-plane. The idea is to drive charge from the bulk to the tip 
apex, which can be done if the polarization is in the direction along the bulk, pointed towards the tip. While there is polarization dependence at any given focal point, this dependence is essentially removed by the fact that any point in the bulk can be focused on; and to achieve a maximum field enhancement only the polarization need be adjusted. Alternatively, any polarization in the yz-plane can be used (with the axes used in Figure 3.1) by merely moving the focus to the location which gives the best signal. This would be the more appropriate approach, since it is not possible to actually see where on the tip the light is focused. Therefore, to utilize this effect, it would be necessary to focus on the tip until a good signal to noise ratio is achieved and then adjust the polarization until the highest signal is found.

Moving the focal volume to point D in Figure 3.1 gives Figures $3.20-3.23$, which show a high background field that would provide poor conditions for high resolution near-field imaging. This point is very similar to point $\mathrm{B}$, in which the incident light is also focused in the same plane as the portion of the sample to be imaged. Just as in point $\mathrm{B}$, point $\mathrm{D}$ shows that focusing in the same plane as the imaging location is not a good idea. These images really drive home the concept that the charge is driven in the direction of the polarization, hence the lobes on the sides of the tip apex and nothing at the end of the tip. From these images, it is also clear that focusing outside of the imaging center plane is crucial to background suppression. 


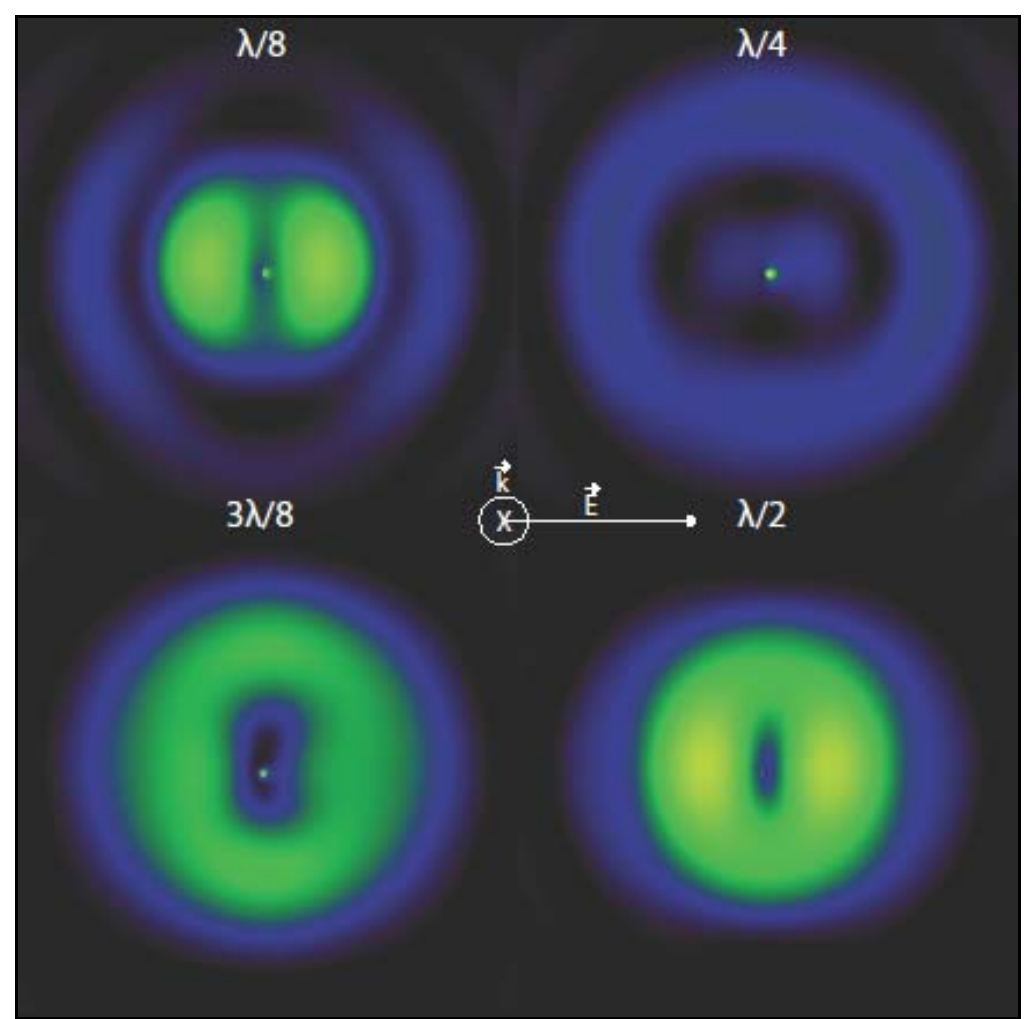

Figure 3.20: The yz-plane field response for a y polarization at point D in Figure 3.1.

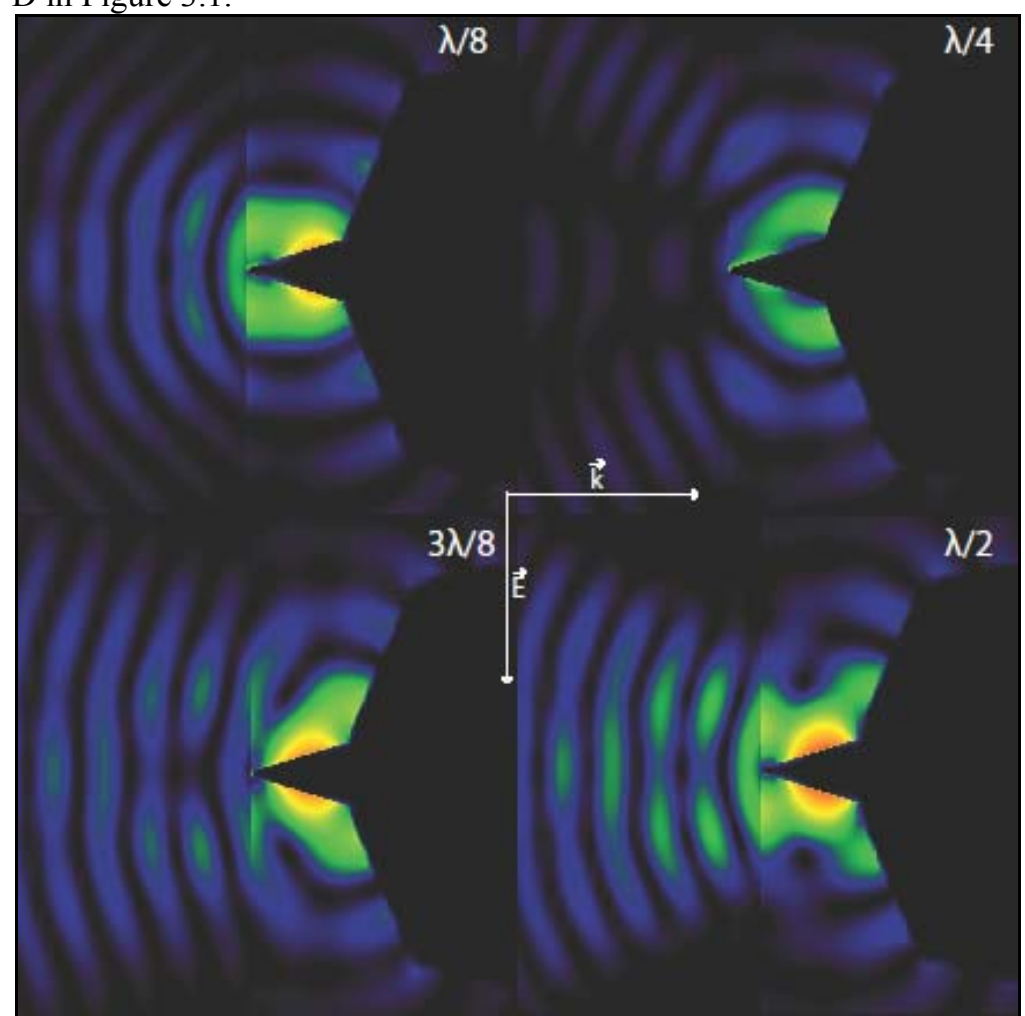

Figure 3.21: The xy-plane field response for a y polarization at point $\mathrm{D}$ in Figure 3.1. 


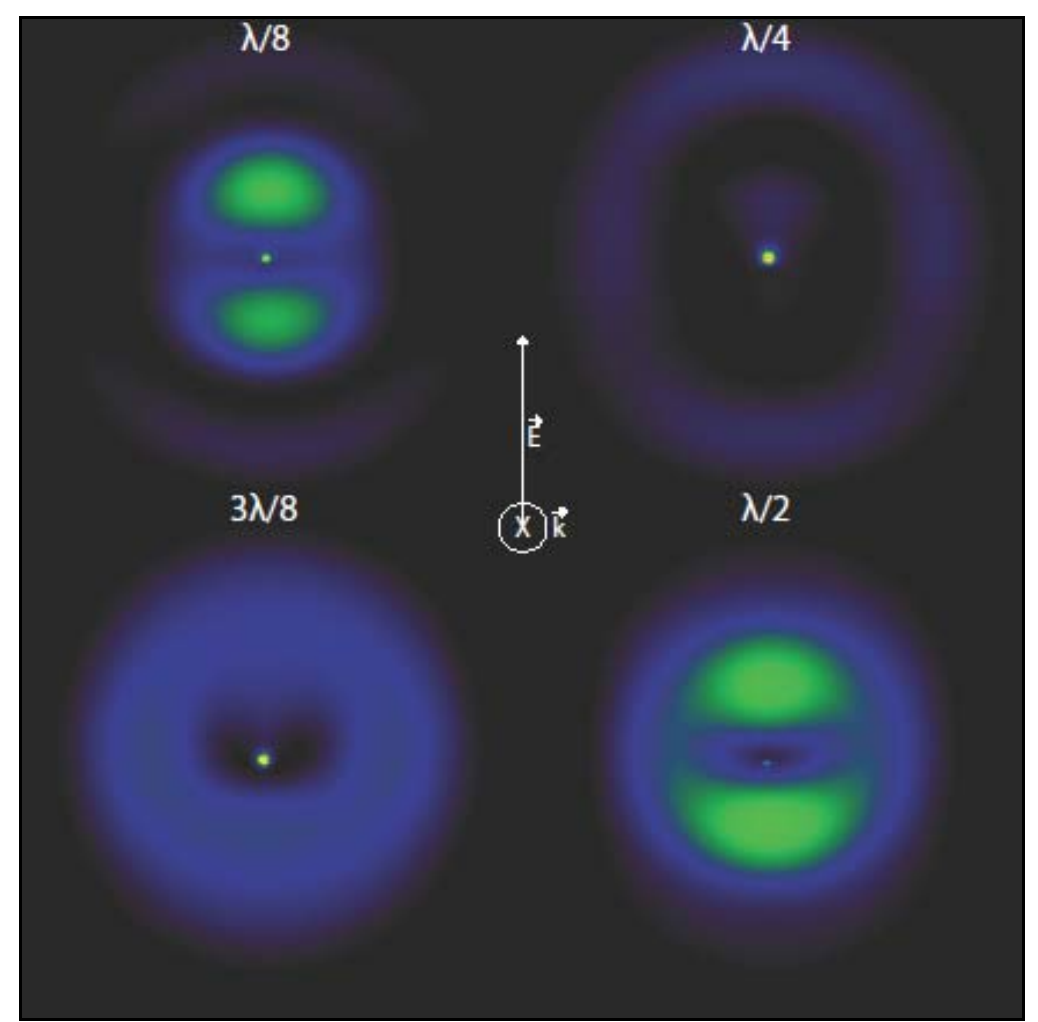

Figure 3.22: The yz-plane field response for a $\mathrm{z}$ polarization at point $\mathrm{D}$ in Figure 3.1.

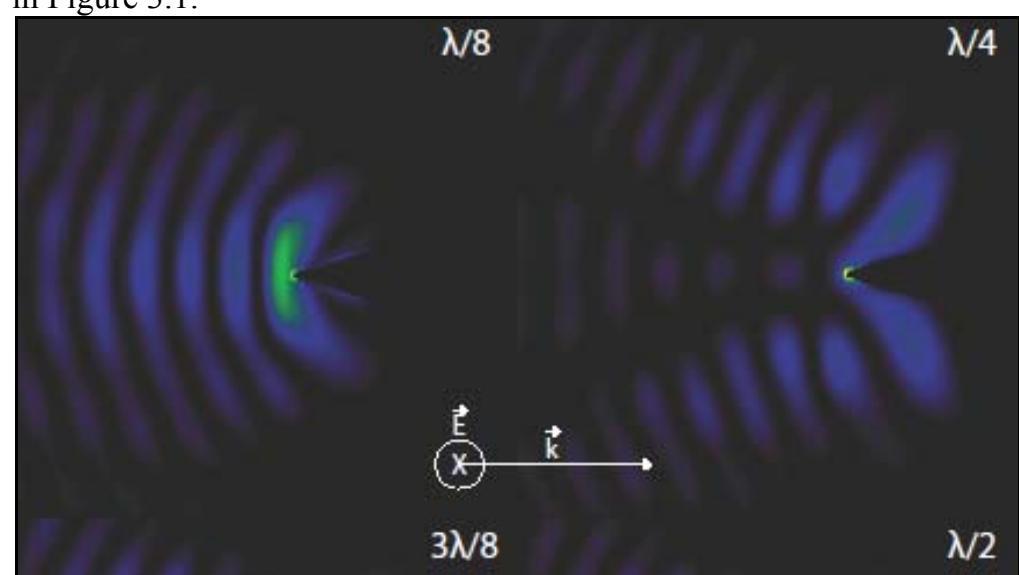

Figure 3.23: The xy-plane field response for a $\mathrm{z}$ polarization at point $\mathrm{D}$ in Figure 3.1. 
There is a slight discrepancy between the two polarizations at point $\mathrm{D}$, shown in Figures 3.22 and 3.22 which remains unexplained. As in point B, the field response should, due to symmetry, be similar in intensity when polarization is rotated. There is a slight difference however, in that the background when the polarization is in the $\mathrm{z}$ direction is slightly lower. This effect is assumed to be some sort of modeling artifact but it remains unclear as to the cause. Due to symmetry the response should be the same in intensity, as is the case with point B.

The final location for the focal volume is at points $\mathrm{E}$ and $\mathrm{F}$, of which only $\mathrm{E}$ is shown, through Figures 3.24-3.27. In these figures, the focal volume is placed away from the imaging center but not on the bulk of the probe. This is, again, a poor imaging scenario, due to the high background signal and the low field at the tip apex. However, it is still possible to see the relationship with polarization. Notice in Figure 3.28 for the $3 \lambda / 8$ snapshot there is a slight field enhancement on the tip apex along the side. This is not in the desired location for near-field excitation but it does show that at least some charge is driven to the tip apex from the bulk. 


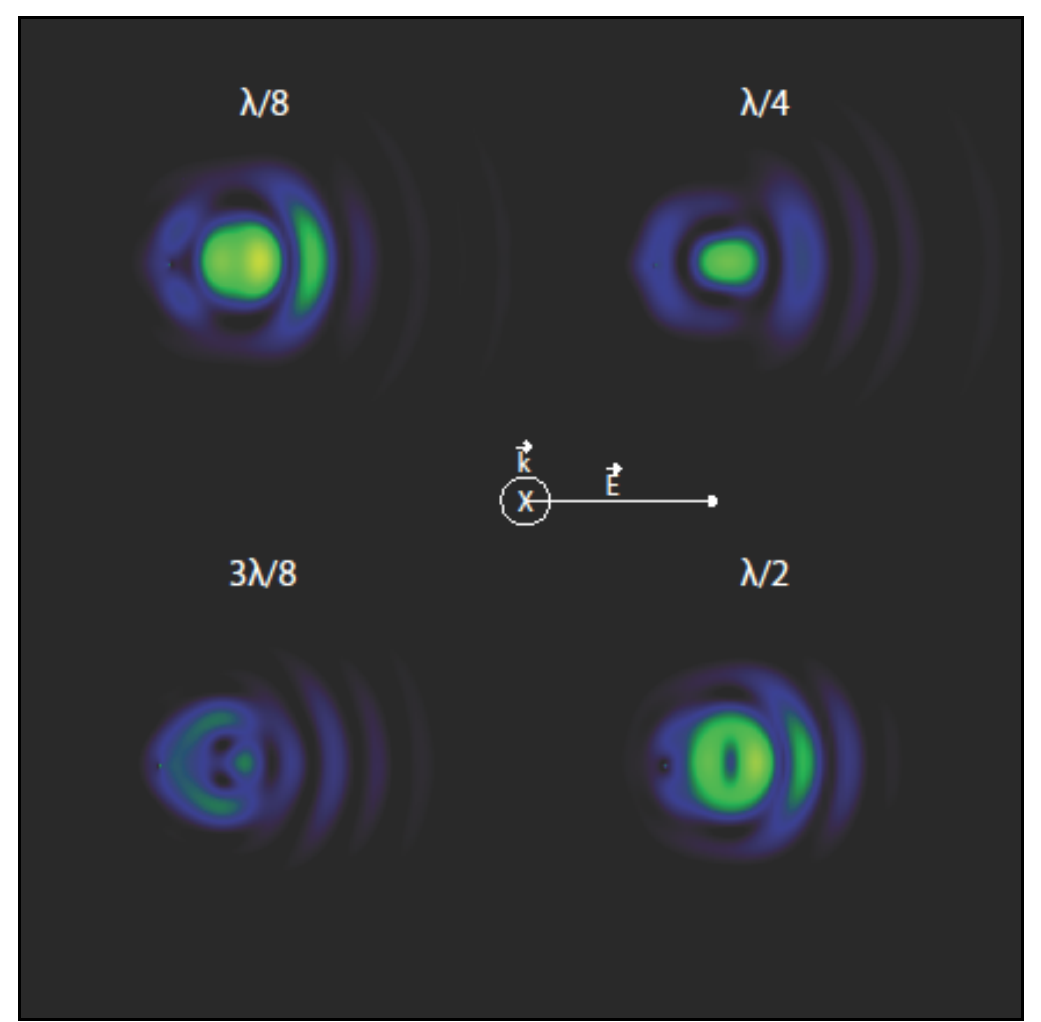

Figure 3.24: The yz-plane field response for a y polarization at point $\mathrm{E}$ in Figure 3.1.

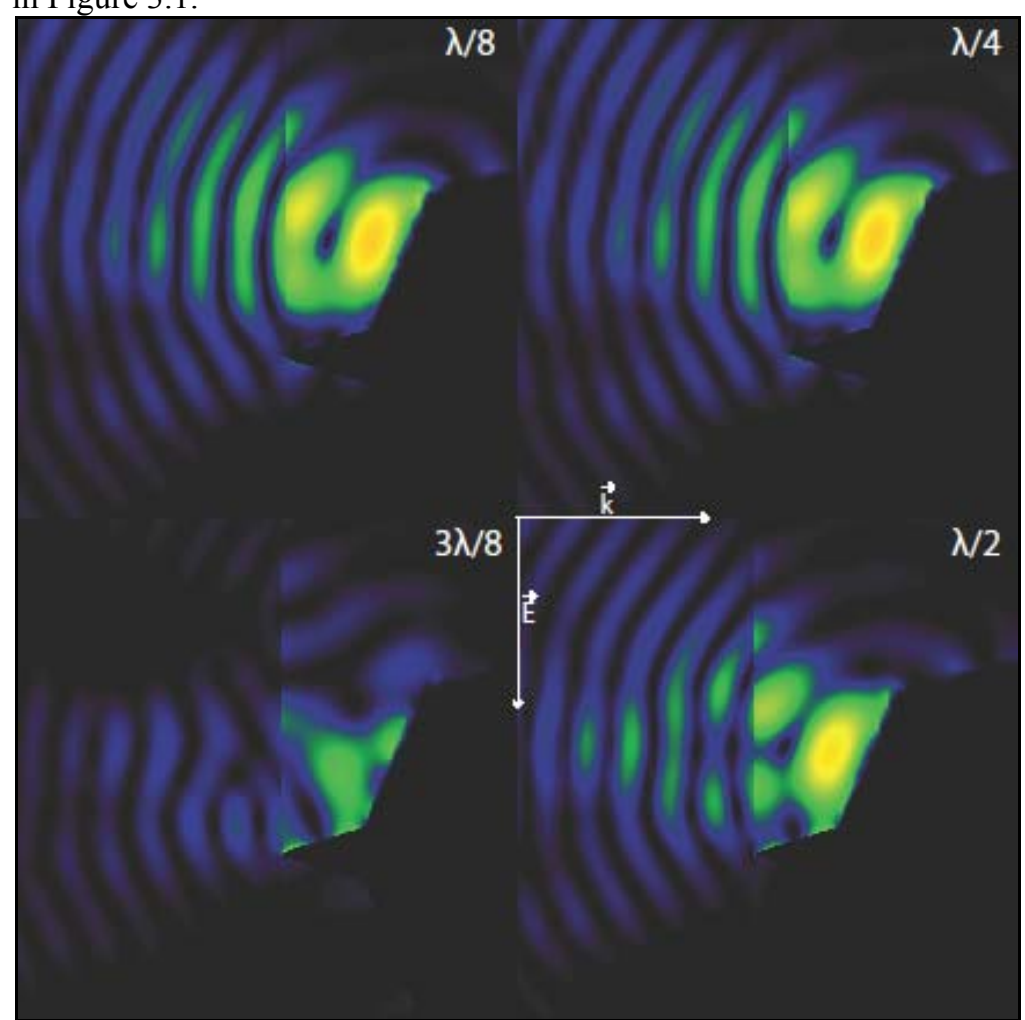

Figure 3.25: The xy-plane field response for a $\mathrm{y}$ polarization at point $\mathrm{E}$ in Figure 3.1. 


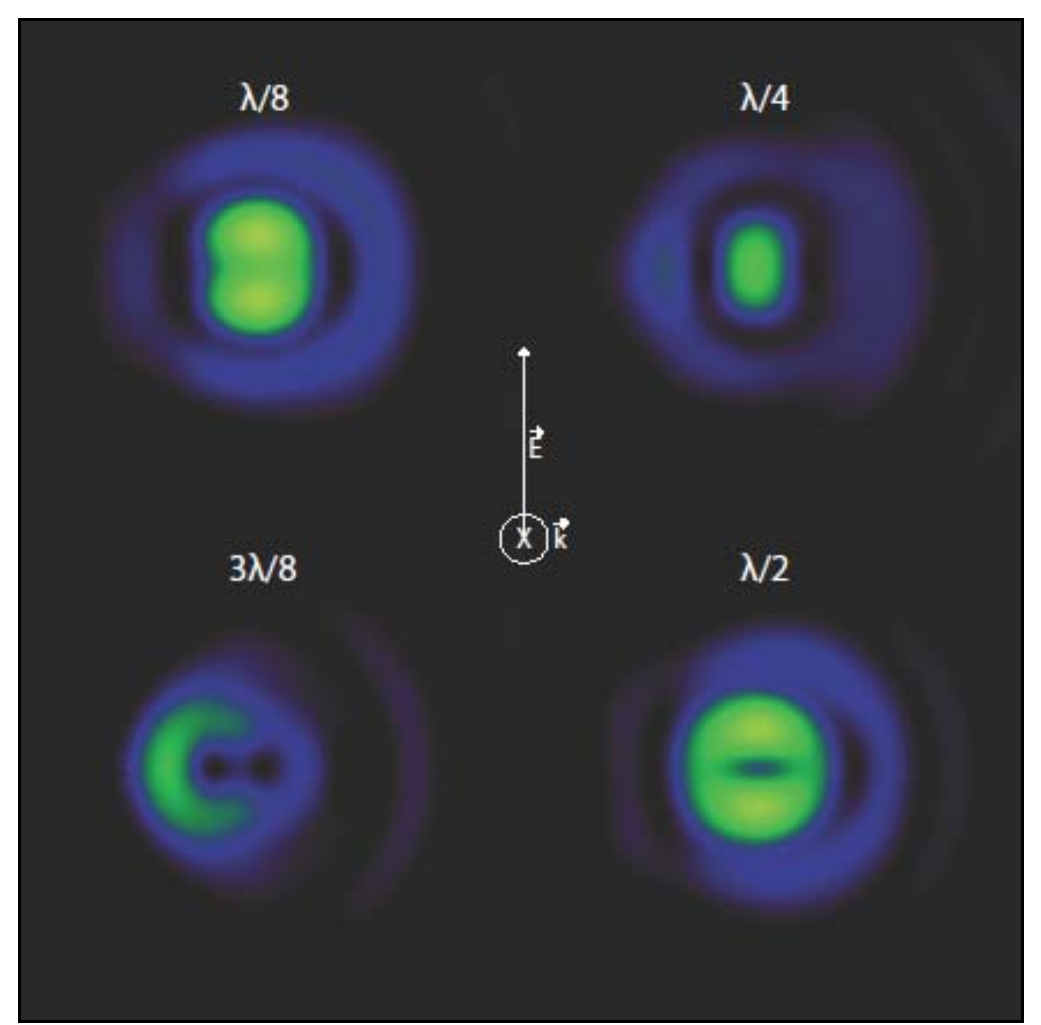

Figure 3.26: The yz-plane field response for a $\mathrm{z}$ polarization at point $\mathrm{E}$ in Figure 3.1.

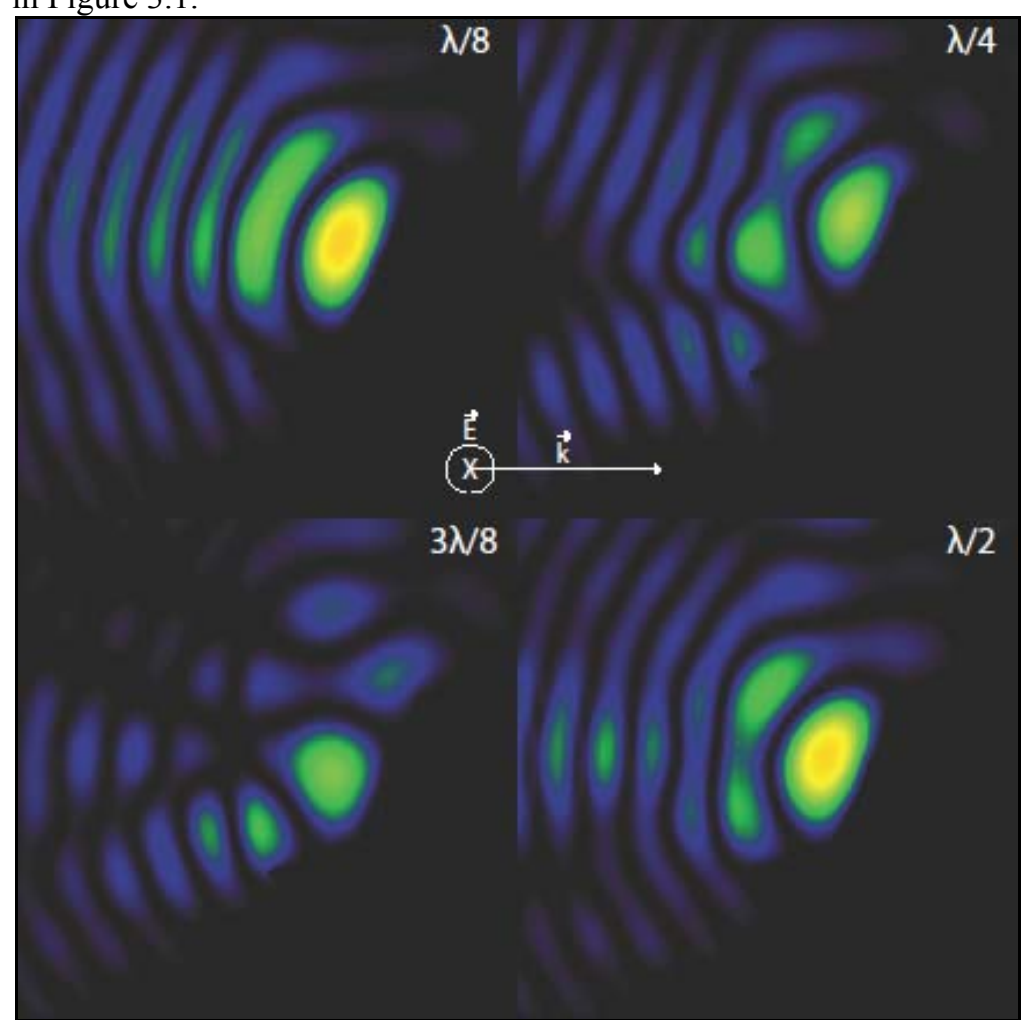

Figure 3.27: The xy-plane field response for a $\mathrm{z}$ polarization at point $\mathrm{E}$ in Figure 3.1. 
The field snapshots throughout this chapter show that the polarization dependence in reality has not been removed. However, any position within the bulk can be utilized as a focal position with equal results, through manipulation of the polarization. Alternatively, any polarization within the xy-plane (as shown below) can be utilized by moving the location of the focus on the bulk. This concept is illustrated in Figure 4.24. At position $\mathrm{P}_{1}$, a polarization in the $\mathrm{y}$ axis is needed, whereas at position $\mathrm{P}_{2}$, a polarization in the $\mathrm{x}$ axis is needed. At position $\mathrm{P}_{3}$, a composite $\mathrm{xy}$ polarization would be needed, shown by the arrow at that location. As shown above, focusing in the bulk region away from the tip apex is key, as any focal locations either in the sample plane or in the tip apex plane will result in very high far-field background, giving a poor signal to noise ratio.

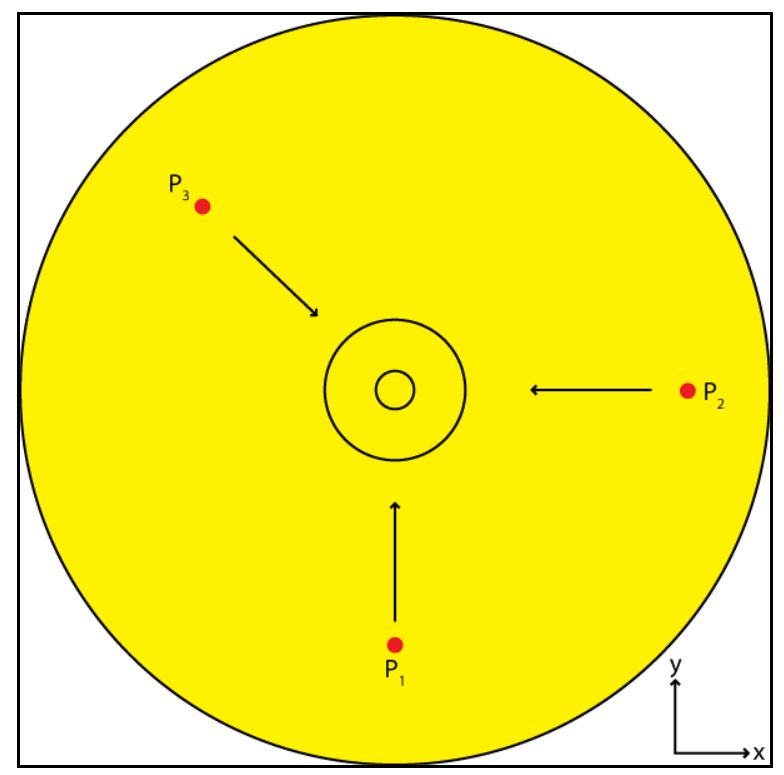

Figure 3.28: Varying location of focal volume or polarization.

The main point here is that it appears that the asymmetric tip would indeed work. With a maximum field of $70 \mathrm{~V} / \mathrm{m}$ at the tip apex for the asymmetric tip modeling, there is an associated field enhancement of about $4,900 \mathrm{~V}^{2} / \mathrm{m}^{2}$ which is very 
large and with background suppression by focusing on the bulk, good near-field imaging conditions will result. 


\section{Chapter 4: Fabrication Methods}

\section{1: Electrochemical etching}

Since the advent of scanning tunneling microscopy (STM), the technique of electrochemical etching has become quite popular in the creation of sub-micron sharp metal tips. Other methods, such as cutting with scissors/razor blade, mechanical polishing, and chemical etching have all been used with varying degrees of success to create STM tips. However, with the advent of near field imaging techniques, the need for not just sharp tips, but sharp tips of specific geometries has arisen. This is something that single step processes just cannot accommodate.

The basis of electrochemical etching is to drive a reaction that would not normally occur, by adding energy in the form of electricity. In the scenario used, gold wire serves as the anode which is being electrochemically etched, a loop of platinum wire serving as the cathode in an electrolytic solution of $37 \%$ hydrochloric acid. The byproducts of this reaction are gold chloride and hydrogen gas. The gold chloride is apparent as a gold color in the solution, which slowly pervades the entire solution. After 10-20 etches with a given solution, the solution is discarded due to contamination by the gold chloride, indicated by the gold color of the solution. The hydrogen gas escapes by bubbling out of the solution, which can cause a problem with reproducibility if the reaction is driven with too high a voltage, as the bubbling becomes so violent that the reaction is not constant throughout the length of the tip [Fotino, 1992].

Most of the literature is focused on the fabrication of Tungsten and Gold probes through the use of DC etching, which has been dubbed the "drop off method". 
This term is due to the way that the reaction occurs at the metal/electrolyte interface, within the meniscus. As the metal at this interface gets smaller, there is a point at which the weight of the portion below the meniscus is larger than that able to be supported by the necked portion, and the lower portion "drops off". One major contributor to probe end radius in a DC etch is the amount of material hanging below the meniscus. Too much material can cause the upper portion to curl up when the lower portion drops off; creating a tip that is useless for scanning microscopy. The literature shows that around 1-2 mm of material below the meniscus produces very sharp tips.

The literature is comprehensive on the use of DC etching for gold and tungsten in order to fabricate STM probes. However, there is a major difference between the fabrication of STM probes and the fabrication of TENOM probes; the idea that perhaps a very sharp tip will not give a good near field imaging scenario, as shown in Chapter 3. Some modifications were therefore made to the etching procedures with an emphasis on obtaining reproducible aspect ratios and surface roughness, as opposed to reproducibly super sharp end radii. The largest modification was the transition to AC etching, which was found to produce the same geometries with a much higher percentage. Figure 4.1 shows the desired geometry which was obtained through AC etching roughly $95 \%$ of the time. This was a very reproducible geometry that worked wonderfully for the purposes of the asymmetric tip fabrication. Both aspect ratio and surface roughness characteristics were excellent for this purpose. 


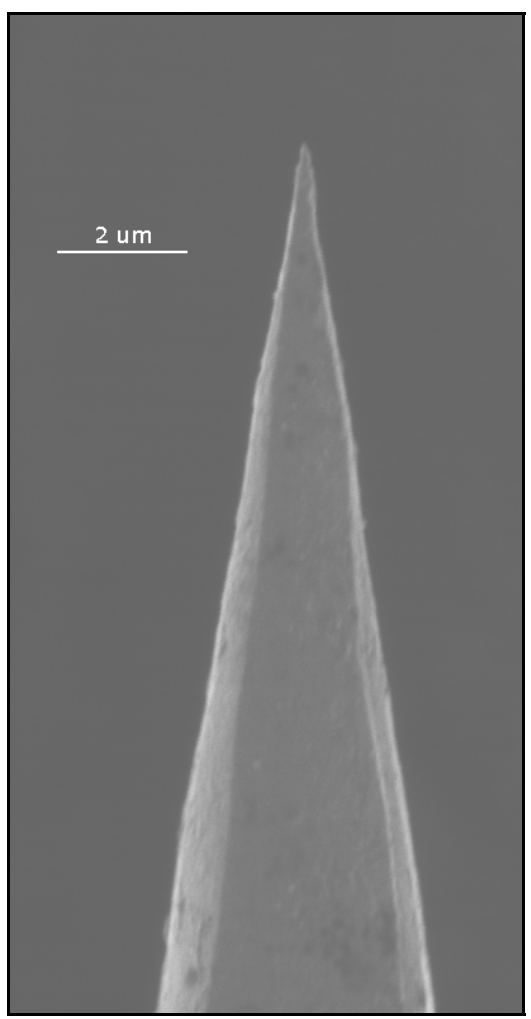

Figure 4.1: A sharp tip, post etch.

\section{2: Focused Ion Beam Systems}

In 1933, a revolution in physics occurred with the invention of the electron microscope by Ernst Ruska. This invention has fueled the technological breakthroughs that society has seen for the past $70+$ years, by allowing physicists to peer deeper and deeper into the nano-world. Utilizing concepts very similar to that of the scanning electron microscope (SEM), the Focused Ion Beam (FIB), is a closely related tool that has pushed the boundaries of technology for the past $\sim 30$ years.

The basis of a FIB system is that heavy ions, usually gallium, are accelerated into a sample, creating an interaction volume like that shown in Figure 4.2. In general, the actual size and shape of the interaction volume is non-trivial in calculation and is left to Monte-Carlo simulations. The beam is scanned across the sample, in discrete 
spatial steps; in a process called rastering. The scan area is divided into a certain number of points, and the beam dwells at each point for a specified amount of time, called the dwell time. The longer the dwell time, the slower the scan and the longer the beam stays at each point, or pixel, in the scan. At each point, secondary electrons or secondary ions are collected to form an image, in a manner very similar to that of the SEM. The major difference between the SEM and the FIB is that due to the much larger mass of the gallium ions compared to the electron, sample atoms are removed, or sputtered from the sample surface.

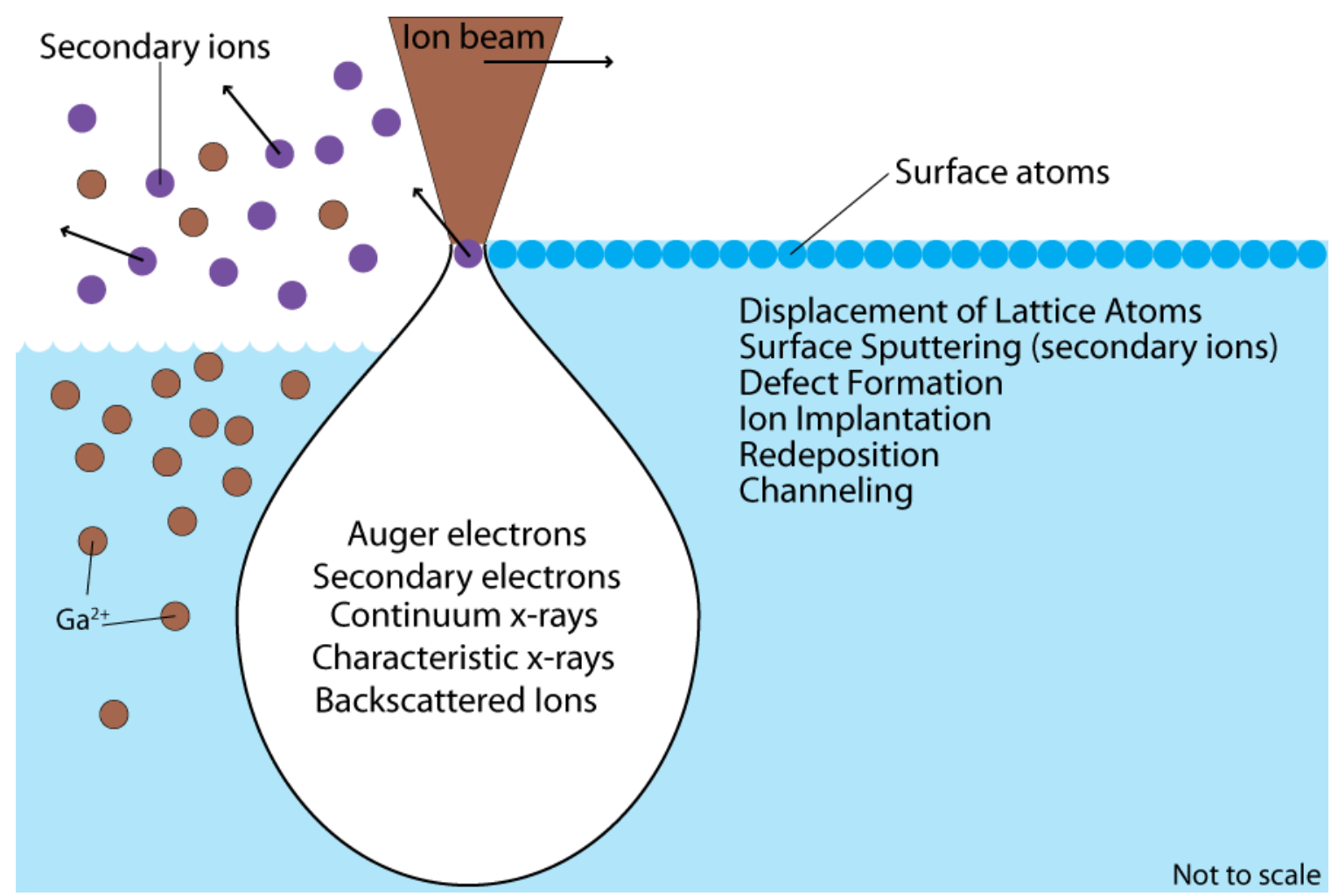

Figure 4.2: A diagram representing the Focused Ion Beam interaction volume.

This sputtering of sample material has a very useful application in that not only can FIB systems be used for imaging but also as an ingenious tool for nanomachining. Since the ions in a FIB are so much more massive than the electrons in an 
SEM, the interaction volume created is much larger, especially at high beam currents and voltages. This allows for the removal of material, in a controlled manner, on a scale much larger than that of an SEM, which is also inherently destructive to samples. This sputtering of sample atoms away from the sample area is one of the major benefits of using a FIB for modification of the sample in the micro or even nano regime. This is the reason why FIB systems are so useful to the modern physicist; they allow for controlled removal of material on the order of nano-scale. The downside to this is that the FIB is inherently destructive to the sample, and at high beam powers, even focusing and stigmating the image is extremely destructive and must be minimized. This destruction can be seen in Figure 5.3, a section of carbon tape. The white square in the center of the image is the location at which the beam had been rastered across for a short period of time. If the magnification was lowered, another square damaged area would be seen in the current image. Basically, the longer the beam dwells on a specific spot of the sample and the higher the beam power, the larger the interaction volume and the more atoms sputtered, resulting in more material removed. 


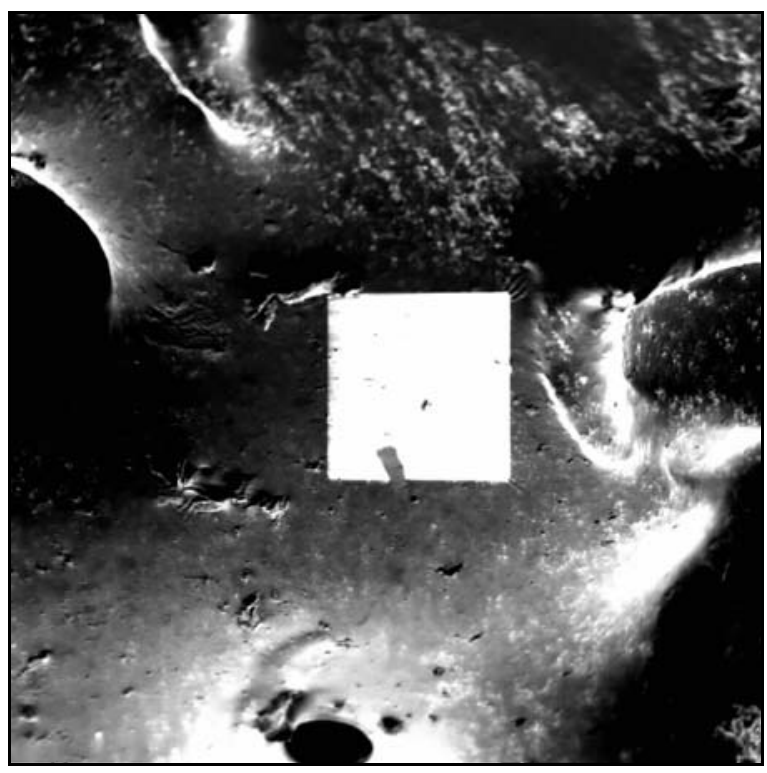

Figure 4.3: The white square shows an area of damage from imaging with the FIB.

One key to imaging in the FIB is to remember that every moment spent on the sample, especially at high magnifications, high beam currents (nanoamps) and high accelerating voltages $(>20 \mathrm{KeV})$, will damage the sample. Generally imaging is done with a low beam current (10's of picoamps), but damage is still very apparent at these low values. Obtaining a high resolution image prior to milling is non-trivial and the beam must be focused / astigmated prior to initiating a milling sequence or the milled feature will be blurred. Also, a longer dwell time will give a crisper image, due to an increase in the signal to noise ratio but the amount of material removed will also increase. This is the concept exploited in this new method of near-field probe fabrication, manipulation of the dwell time at each pixel that the beam focuses on while milling. This is done automatically, as outlined in section 5.5.

One major problem of FIB machining is the deposition of Gallium atoms on the sample surface and the redeposition of sputtered atoms elsewhere on the sample surface. Redeposition is a major hurdle, especially when milling deep, narrow 
features in a material. This is a line of sight phenomena, where sputtered sample atoms leave the surface but are then deposited back onto the surface at a different location. Due to the geometries being worked with, the lack of high aspect ratio holes and the line of sight leading from the probe directly to vacuum, redeposition did not appear to be a problem. Gallium deposition is a potential problem, as it would affect the material parameters of the tip which could in turn affect the field at the tip apex, but this was not investigated.

\begin{tabular}{|l|l|l|l|l}
\hline$s$ \\
40 \\
25 \\
96 & 1229 & 2867 \\
96 & 1639 & 2867 \\
96 & 2048 & 2867 \\
96 & 2457 & 2867 \\
96 & 2867 & 2867 \\
96 & 1229 & 2457 \\
96 & 1639 & 2457 \\
96 & 2048 & 2457 \\
96 & 2457 & 2457 \\
96 & 2867 & 2457 \\
96 & 1229 & 2048 \\
96 & 1639 & 2048 \\
96 & 2048 & 2048 \\
96 & 2457 & 2048 \\
96 & 2867 & 2048 \\
96 & 1229 & 1639 \\
96 & 1639 & 1639 \\
96 & 2048 & 1639 \\
96 & 2457 & 1639 \\
96 & 2867 & 1639 \\
96 & 1229 & 1229 \\
96 & 1639 & 1229 \\
96 & 2048 & 1229 \\
96 & 2457 & 1229 \\
96 & 2867 & 1229 \\
9
\end{tabular}

Figure 4.4: An example of a stream file.

\section{3: Stream files}

A stream file is an ASCII text or binary file which gives a set of instructions to the FIB. These files interact directly with the patterning data acquisition card (DAC) of the FIB, which gives a patterning area of 4,096 in $\mathrm{x}$ by 3,536 in $\mathrm{y}$. Stream files are proprietary to FEI systems and vary slightly between different models. Micrion systems have similar functionality with the MDDL Job File, but are much more difficult to work with, as they are not simple text files.

Stream files work by assigning dwell times in tenths of microseconds to specific $(\mathrm{x}, \mathrm{y})$ coordinates. The dwell times range from $.1 \mu$ s to $4.6 \mu \mathrm{s}$ in an approximately logarithmic scale with 120 values. This provides functionality in patterning beyond the simple 
primitives included in FIB software packages. Even milling in such a simple geometry as a disk with a hole in the middle (donut) can be impossible without the use of stream files. Each stream file contains a specific 3 line header. This header changes between models, as it is specific to the version of software the FIB is using. For XP 3.35 versions, the header is as follows. The first line is the letter "s", to denote a stream file. The second line is the number of times to repeat the pattern. The third line is the number of total $(x, y)$ coordinates within the stream file. Figure $x$ shows an example stream file, from the XP software manual, containing 25 points on which to dwell, with the pattern repeated 40 times.

\section{4: Automation through LabVIEW ${ }^{\mathrm{TM}}$}

LabVIEW is a graphical user interface (GUI) based development package created by National Instruments, utilizing the graphical programming language "G". LabVIEW is short for Laboratory Virtual Instrumentation Engineering Workbench. Programs are known as VI's or "Virtual Instruments" which are generally made for interfacing hardware with software. It is designed to easily send commands to hardware through a data acquisition card. LabVIEW's greatest strength is that one or two icons, known as function blocks, can represent many pages of code, making it a very efficient way of programming.

LabVIEW utilizes two different interfaces to create a VI, the block diagram and the front panel. The block diagram is where all of the actual programming takes place. In the front panel the user interacts with the program including controls, inputs and output displays LabVIEW is different from syntax based programming 
environments in that rather than stringing together lines of code; the programmer connects pictographic function blocks with wires. To place function blocks, it is as simple as drag and drop. These function blocks are then wired together. Each function block acts as multiple lines of code and generally has multiple inputs and/or outputs. The idea is to simplify the process of programming for an individual with little to no knowledge of syntax based programming.

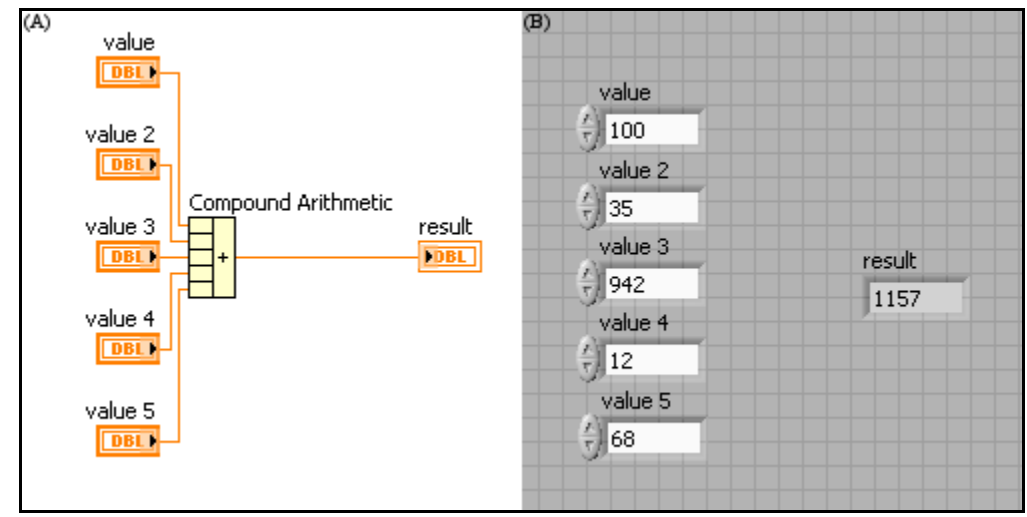

Figure 4.5: An example VI, including both block diagram (left) and front panel (right).

As an example, a very simple VI is used to add five numbers. Both block diagram and front panel is displayed in Figure 4.5. In this VI, values 1-5, which are set in the front panel, are wired into the compound addition function block. The output of the compound addition block is wired to an indicator on the front panel, displaying the result. In practice, any useful VI is going to be much more complicated than this, which is why it has been decided to not include the actual block diagram. One helpful aspect of LabVIEW is that error checking is done in real time while programming, which is a very nice convenience when dealing with the graphical complexity of most VI's (much like a complex circuit diagram). 


\section{5: Pixmaps}

A pixmap is a type of file which, in an image, stores a numerical value representing a color at every pixel location. A pixmap contains $2^{\mathrm{n}}$ colors per pixel, in an n-bit image. Therefore, an 8 bit image contains a range of 256 colors per pixel, from 0 to 255 . This can be an actual color scheme or grayscale when dealing with anything less than and including 8 bit. 8 bit grayscale bitmaps were used extensively, with 0 representing white and 255 as black. Values in between 0 and 255 are scaled accordingly; thus a value of 10 would be very close to white, while similarly, a human eye could not tell the difference between a value of 245 and 255. A value somewhere around 130 would be considered neutral gray.

Bitmaps are a subset of pixmaps, having a depth of only 1 bit, or a color of black or white. However, there is a pixmap file format specifically called Windows Bitmap, or .bmp, which was used exclusively. The name is a bit misleading, because, as mentioned, a bitmap is actually only a 1 bit image, but a .bmp can be up to 32 bit. In the following discussions, all reference to the term bitmap will be used to describe the Windows Bitmap file format, or .bmp. A .bmp file contains more information than just the pixel data. It includes a header and information about the file itself, in addition to a color palette for assigning actual numerical values to colors. Within the actual file data, there is included a color value for each pixel location. However, all information contained in the bitmap aside from the actual data and the size of the image in pixels, is unnecessary and discarded. 


\section{6: Creating stream files from bitmaps}

One of the most critical aspects of this fabrication method is the creation of stream files. These stream files are what give the FIB the functionality needed to create complex geometries. However, it would be nearly impossible to create any kind of realistically useful stream file by hand, due to the sheer number of points. This makes the idea of creating stream files from .bmp's a desirable alternative. Fortunately, software drawing programs are both numerous and straightforward in implementation. Therefore, it is much easier to create a .bmp with any capable image creation software (something even as simple as Paint, which comes with Windows) and then create a stream file from the .bmp than it is to create a stream file of the desired pattern by hand.

For the purpose of creating a stream file from a bitmap, a VI was created in Labview version 8.6. This VI, when started, prompts the user to input the path to a .bmp file. This file is then analyzed by the VI. This process removes all information from the file, except the actual data and size of the file, in pixels. The pixel data is then turned into a column array of dwell times. These dwell times, as previously mentioned, are given a value between $.1 \mu$ s and $4.6 \mu$ s, distributed approximately logarithmically across 120 values. The program also allows for adjustment of the output dwell times, to allow different possible distributions of dwell times for flexibility. This allows for precise control over the dwell times associated with different grayscale values, which means it is possible to obtain variability in the range of dwell times used. Therefore it is possible to give a pixel value of 1 a dwell time of .1 $\mu$ s and a pixel value of 255 a 
dwell time of $4.6 \mu \mathrm{s}$. However, it is just as possible to assign a pixel value of 1 to be a dwell of $3 \mu$ s and a pixel value of 255 , a dwell of $4 \mu$ s.

The size data is used not only to create the header for the stream file, but also the $\mathrm{x}$ and $\mathrm{y}$ coordinates for each position. Since the VI reads pixel data from the top left to the bottom right a counter is used to count from 0 to the maximum $\mathrm{x}$ value for every value of $y$ in the image. Therefore, in a $2 \times 2$ image, the output would be $4(x, y)$ coordinates: $(0,0),(1,0),(0,1)$ and $(1,1)$. Both $\mathrm{x}$ and $\mathrm{y}$ are created separately in their own column arrays. The $\mathrm{x}$ array will count from 0 to the maximum $\mathrm{x}$ value and then repeat until the total number of points has been counted. This is different than the $y$ array, which will count each value $\mathrm{x}_{\max }$ times, and then move to the next value, counting each $\mathrm{y}$ value $\mathrm{x}_{\max }$ times until $\mathrm{x}_{\max } * \mathrm{y}_{\max }$ values have been counted. This total number of points is placed into the header (line 3). A flowchart of the program can be seen in Figure 4.6.

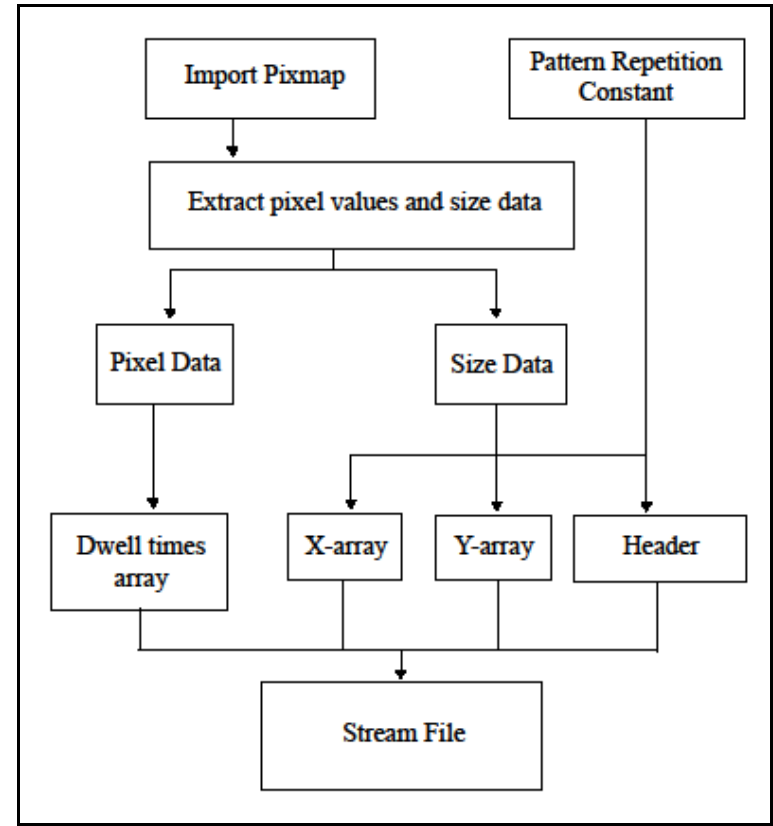

Figure 4.6: The process by which the LabVIEW VI converts a bitmap into a stream file. 
In order to maximize the number of points we are able to use in the bitmap, all white pixels, which are given a value of 0 for dwell time, are removed from the stream file. Thus white pixels are not dwelled on. This concept can be seen in Figure 4.7, which is a testing bitmap and its corresponding stream file from the LabVIEW VI. The bitmap itself consists of 4 pixels: 1 black, 1 white and 2 shades of gray. As can be seen by the resulting stream file, the VI reads the bitmap from the top left to the bottom right, and removes all white pixels. The stream file has a header that indicates a pattern consisting of 3 points, each repeated 10 times. The $\mathrm{x}, \mathrm{y}$ coordinates do not, however, start at $(0,0)$. This is to keep the pattern centered on the FIB software screen.

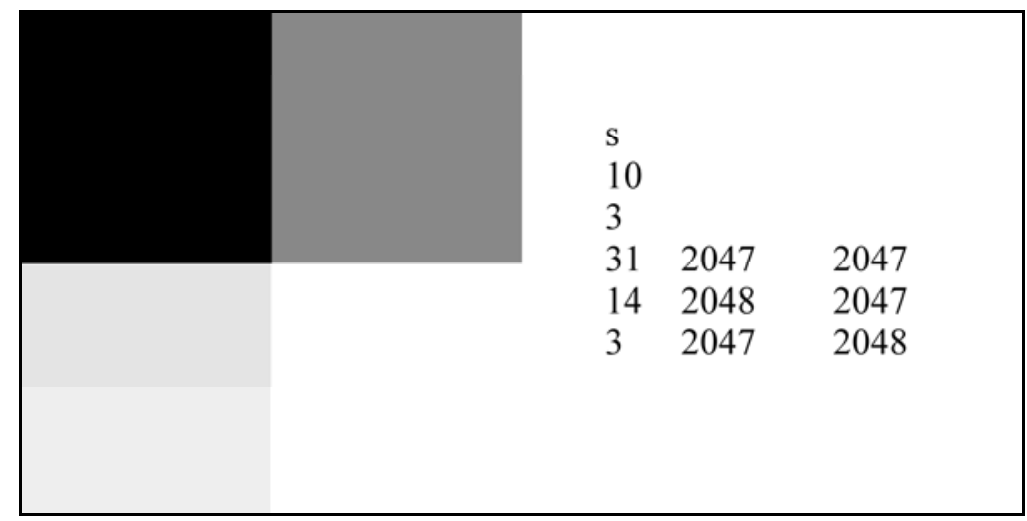

Figure 4.7: A four pixel bitmap and its corresponding stream file.

In reality, the above file would be much too small to be of any use. The FIB rasters over 4,096 pixels by 4,096 pixels, meaning that a 2 pixel by 2 pixel image would be very small, even at extremely high magnifications. This is due to the fact that the pattern remains the same size in square pixels no matter what the magnification, as it is specified by a certain number of pixels, which remains fixed at varying magnifications. 


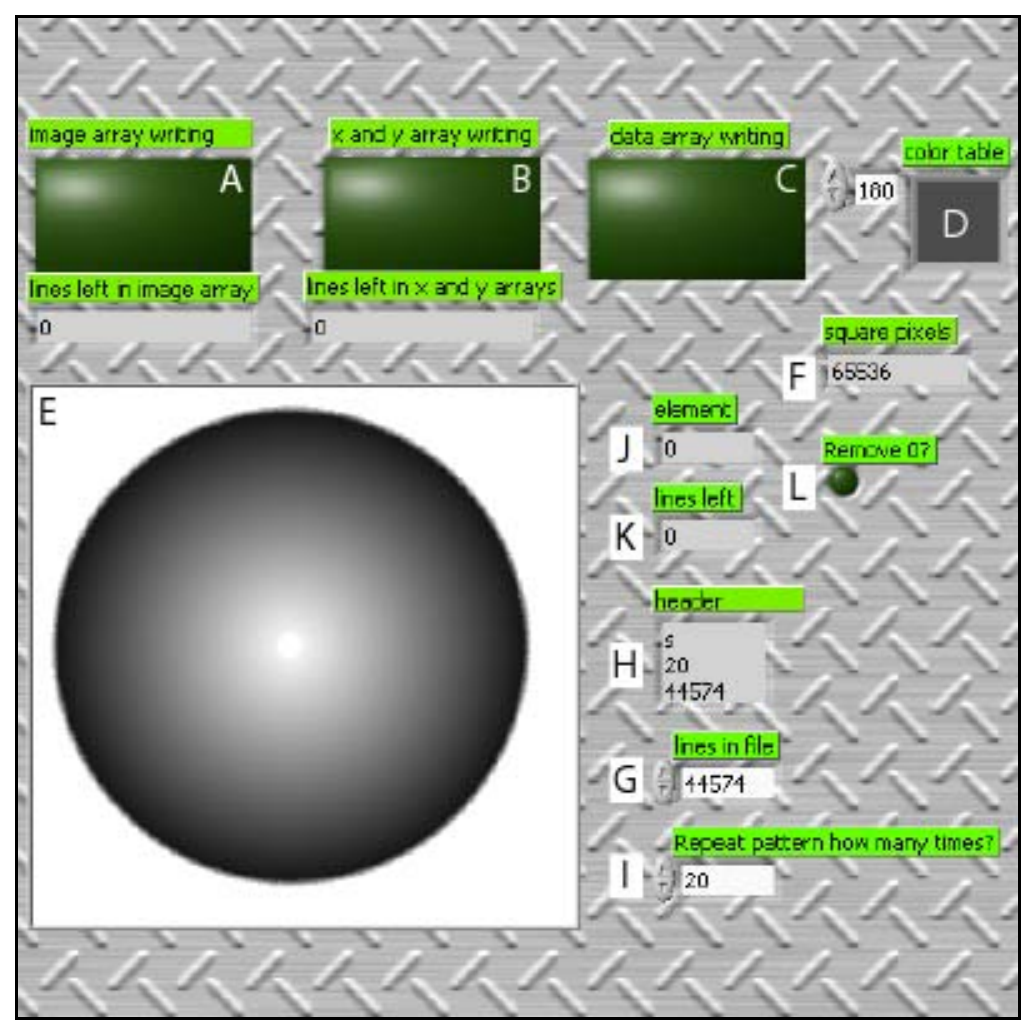

Figure 4.8: The graphical user interface for the VI.

The front panel of the VI is displayed in Figure 4.8. The 3 large boxes are LEDs that correspond to the processes taking place. The leftmost LED (A) indicates when the VI is writing the image array, or the actual pixel data. The middle LED (B) denotes the creation of the $\mathrm{x}, \mathrm{y}$ position arrays. The rightmost LED (C) represents the combination of the previous 2 arrays into the array that will create the stream file. To the right of the LEDs is the color table (D), displaying the grayscale colors and the corresponding value between 0 and 255. The main display (E) in the lower left of the front panel is an image output of the bitmap that the VI is converting to a stream file. The "square pixels" box (F) is the total number of pixels in the bitmap, while the "lines in file" box $(\mathrm{G})$ represents the number of pixels in the output stream file. The "lines in file" box is also inserted into the third line of the header, as can be seen in the 
"header" box $(\mathrm{H})$. The second line of the header is, as expected from the earlier discussion on stream files, the value from the "repeat pattern how many times" box (I), which is how many times the pattern is to be repeated by the FIB. The "element" $(\mathrm{J})$ and "lines left" $(\mathrm{K})$ represent the value of the current pixel being written and how many pixels are left; during data array writing. The "remove 0" LED (L) will light up whenever the "element" value is 0 and that element is not written to the data array. This is the point at which all white pixels are removed from the stream file.

\section{7: Bitmaps used}

All bitmaps created for this project were made using the GNU Image Manipulation Program, or GIMP, which is an open source alternative to Adobe's Photoshop. The creation of a good bitmap is a crucial step for successful probe fabrication, since the probe is made directly from the bitmap. For most of the work

performed, two geometries were used, one for testing and proof of concept on flat substrates and one for actual runs on etched gold tips. These can be seen in Figure 4.9, with the test bitmap on the left. The reason for using this bitmap is for verifying the concept on flat substrates. The darker areas are milled more than the white areas, which should, in theory, result in a conical shape with a small protrusion in the center. This geometry would also work well with an etched tip that has been milled flat at around 2 microns. The hole in the center of each bitmap is designed to give a $100 \mathrm{~nm}$ tip apex if the diameter of the bitmap is 2 microns. 


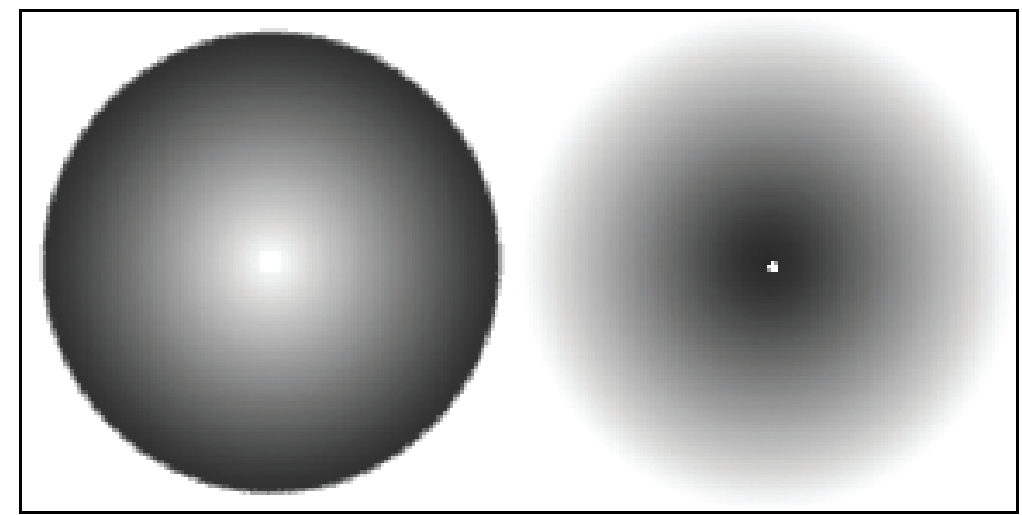

Figure 4.9: The two bitmaps used in the fabrication process.

The geometry on the right of Figure 4.9 is more designed for curved surfaces; however there are some difficulties which arise when dealing with these substrates. The curvature resulting from the etching process is different every time, so no mill is directly the same as any other. This makes repeatability difficult. For this reason it is thought that perhaps the first step should be to cut the tip flat at a 2 micron end diameter and then use the left geometry from Figure 4.9. In order to use the right hand geometry, it would be necessary to modify it for each separate tip, modifying the rate of change from black to white, depending on the curvature of the tip. This does not sound reasonable for an automated process, so again, it is recommended to first cut the tip flat at 2 microns diameter and utilize the left hand geometry.

\section{8: Experimental Procedures}

This section details the exact procedure for creating the symmetric tip geometry, including the correct parameters for each step. Before beginning the process, it was found that a bit of preparation was useful, in the form of sample stub creation. The FIB used was a Strata DB-237 FEI dual beam FIB/SEM which means that while the electron beam is incident, the ion beam is not at a right angle to the sample plane. 
This creates a need for some planning in the form of stub development. Since the ion beam forms an angle of $38^{\circ}$ with the sample, a stub with an angle of $52^{\circ}$ needed to be created, in order to be able to mill the tips head on. Since the dual beam used also contains a STEM (Scanning Transmission Electron Microscope) detector, it is desirable to make the angle slightly shallower than normally necessary, as tilting the stage in the direction of the STEM detector has a smaller range than away from the detector. This gives slightly more room to tilt the sample in case the probe was not mounted correctly. Due to these considerations, sample holders were manufactured from small lengths of aluminum rod, in a way that makes it easy to rotate the probe tip orthogonal to the beam. Originally it was thought that getting the probe head on would be a very difficult process and in the older FIB used (FEI 611) it was indeed difficult, due to the lack of precision in the stage. However, in the DB-237, it was not difficult by any means, except for the caution required in not damaging the STEM detector inside the chamber. The key is to get the tip so that it is exactly orthogonal to the plane of the beam, so that the probe is milled straight on.

The first step involves, as mentioned above, electrochemically etching a length of gold wire (100 $\mu \mathrm{m}$ diameter). It was found, again as mentioned above, that $\mathrm{AC}$ etching will produce more reproducibly smooth, good aspect ratio tips and so an AC etching circuit is used, as pictured in Figure 4.10. 


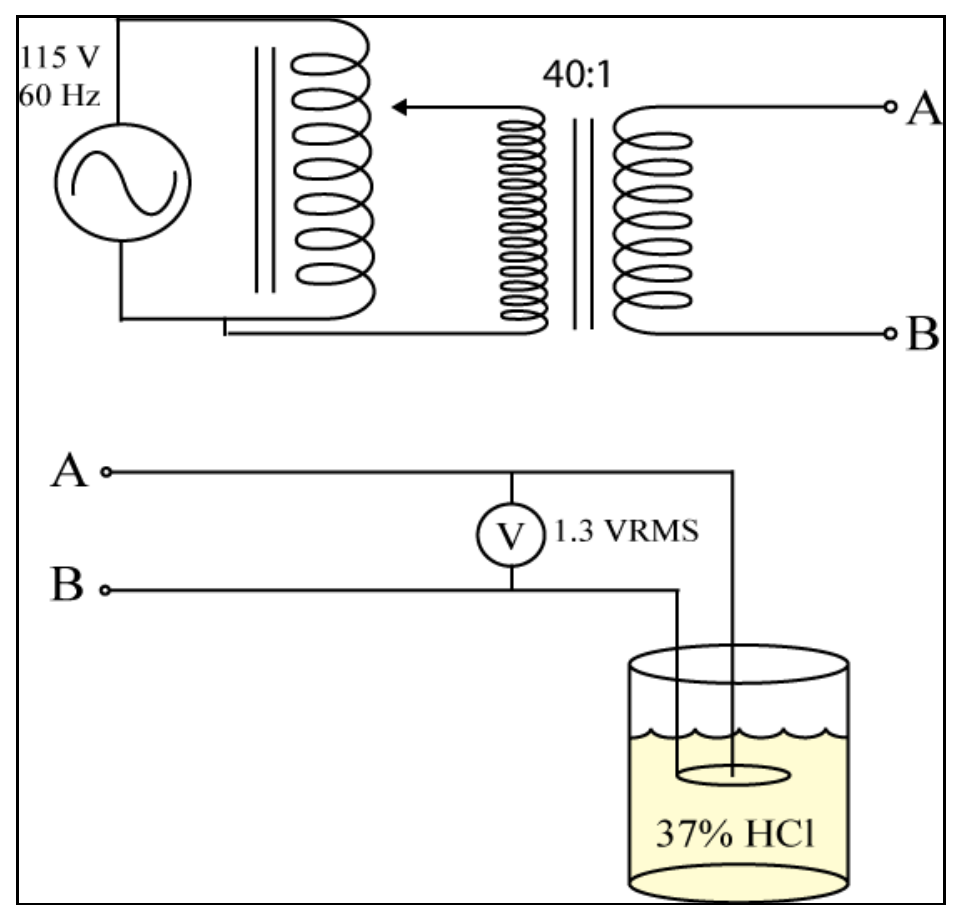

Figure 4.10: The etch circuit used in the electrochemical etching step of the fabrication process.

In this circuit, a normal wall outlet $(115 \mathrm{~V})$ is used as the primary source, which is connected to a high current (10 A) variable transformer (General Radio Type V10), allowing for control of the AC voltage entering the step-down transformer, which brings the voltage down to the range generally reported in the literature for $\mathrm{AC}$ etching of gold $(<10 \mathrm{~V})$. This step down transformer is a Stancor model p-3064 which has an input voltage of $115 \mathrm{~V}$ with an output of $3.15 \mathrm{~V}, 6 \mathrm{~A}$, allowing for a high power output to the setup. This setup was originally a self contained unit, with the current variable transformer replacing a Type 10B powerstat, which only has an ouput current of 1.75 A. This fixed a myriad of reliability issues from complete unreliability to extreme surface roughness. The etch itself is conducted, for a reliably smooth surface and a good aspect ratio, with a voltage at or around 1.3 VRMS. Again, the key to the reproducibility appears to be to not let the process become current limited, so careful selection of the correct transformers is necessary. 
There are a few concerns with etching when trying to get reproducible results. It is advisable to always insert both the gold wire and platinum counter-electrode to the same depth in the $\mathrm{HCl}$. The gold should not protrude beyond the bottom of the counter-electrode. The depth to which both are inserted is slightly variable; however, insertion of roughly $2 \mathrm{~mm}$ below the bottom of the meniscus gave good results. This is generally done using a micrometer. Another concern with the etching is vibration, especially if doing a DC etch. As mentioned above DC etching follows the "drop off" method, and occurs at the meniscus/tip interface. Therefore vibrations would cause the meniscus height to vary adding another, random, variable to the process. This would also occur with $\mathrm{AC}$ etches because the reaction occurs throughout the solution, so varying meniscus height would effect the tip above the level of the solution. It is necessary to also keep all electrodes free from corrosion (difficult when working with $\mathrm{HCl}$ ), as this creates an inconsistent electrical connection and adds a variable to the process. Very fine sandpaper is helpful for keeping electrical contacts clean, although after many etches, the contacts will need to be replaced. For this reason, low-cost copper alligator clips are used in the etch circuit to hold the cathode and anode in the solution.

The next step after etching is to begin making cuts with the FIB. As mentioned above it is likely best to first make a cut at a right angle to the tip, forming a flat region roughly $2 \mu \mathrm{ms}$ in diameter. After this first cut, it is necessary to bring the tip into a head on view. This step is one of the most crucial to the whole process, as the bitmap must line up exactly with the flat portion of the tip, or the effects shown in Figure 4.11 or 4.12 can result, where the cut made is off-center or tilted. 


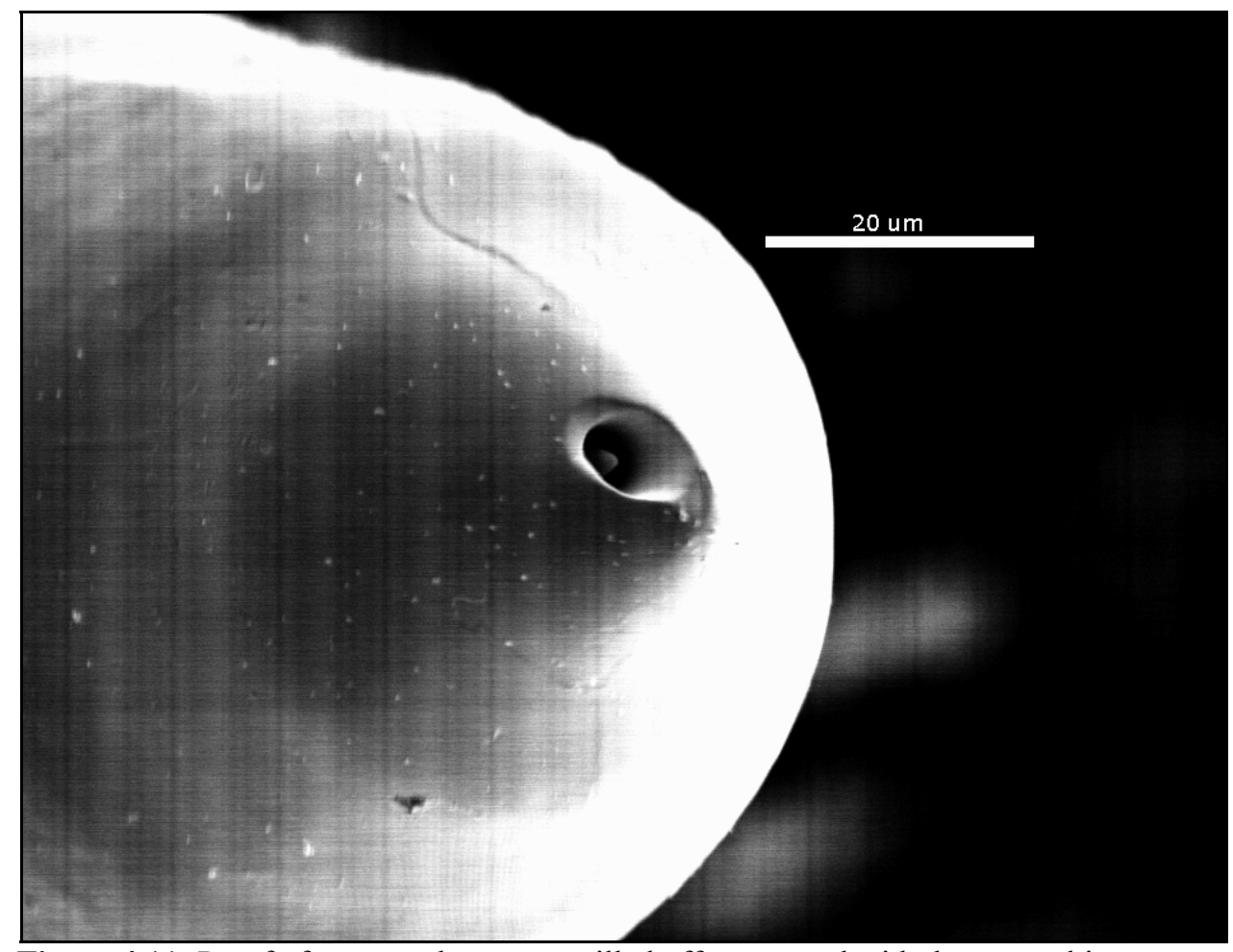

Figure 4.11: Proof of concept, however, milled off center and with the wrong bitmap. The tip inside the hole is $\sim 500 \mathrm{~nm}$ in diameter. This is very preliminary.

Generally, imaging with the FIB should be minimized and use of the electron

beam maximized, however, this can be difficult, especially if not at eucentric height, where the two beams are coincident. When using the FIB to image, a very low beam current should be used to minimize sample damage. It was found that $10 \mathrm{pA}$ was the best compromise between image clarity and lack of damage to the probe. For milling a current of $100 \mathrm{pA}$ can be sufficient, although this is somewhat low for a large volume of material removal. Currents of up to $5,000 \mathrm{pA}$ were investigated, with no clear answer as to which is the best for a general situation. The problem with high currents is the possibility of milling too far, as in Figure 4.12, where the probe is utterly destroyed. Notice the sub-100 $\mathrm{nm}$ tip in the center of the blasted region, showing the concept can indeed produce a sharp tip. 


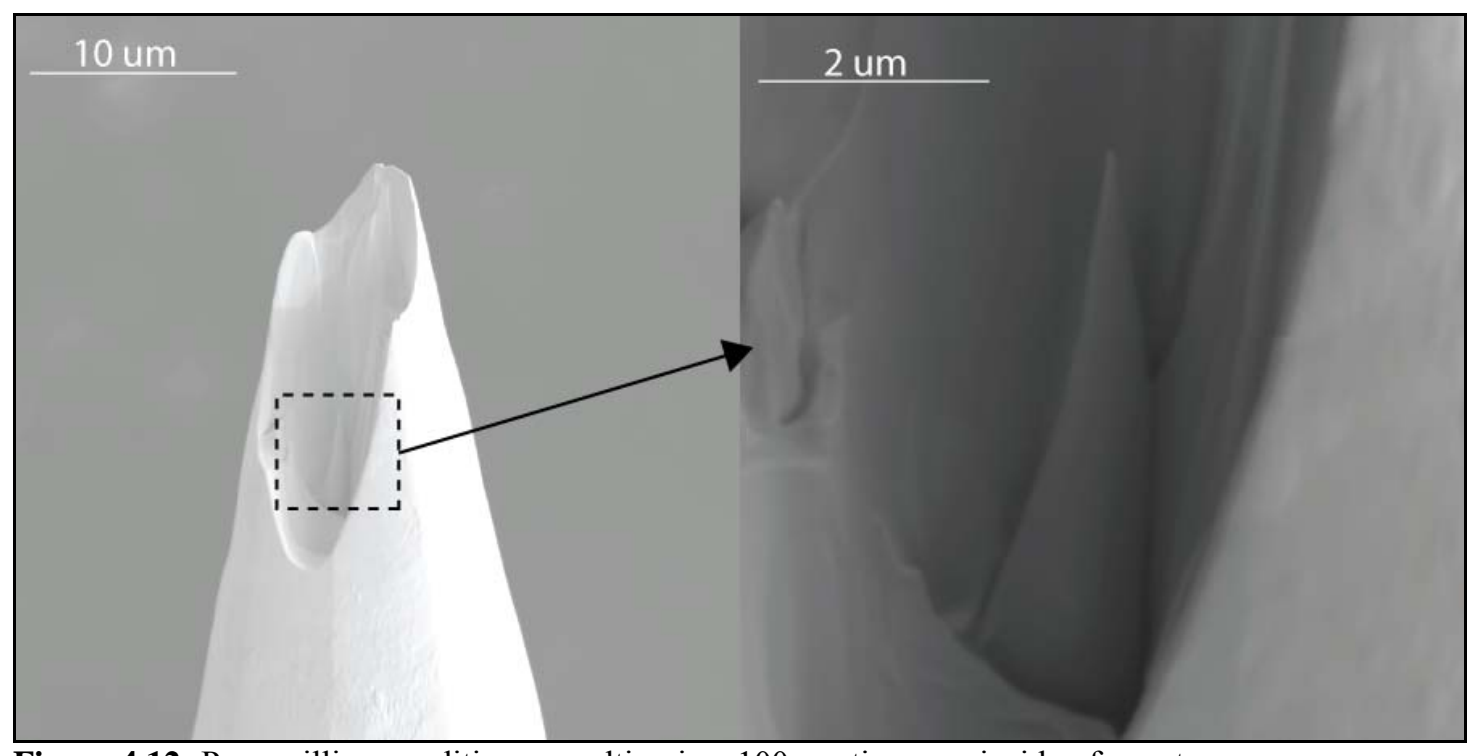

Figure 4.12: Poor milling conditions, resulting in a $100 \mathrm{~nm}$ tip apex, inside of a crater. 


\section{Chapter 5: Discussion and Conclusions}

\section{1: Discussion and Conclusions}

It has been shown through FDTD modeling that with the symmetric probe design there is a very real benefit in the possibility of utilizing any polarization within the sample plane, by moving the focal location. Another benefit is, as in the asymmetric design, the ability to remove the effects of the far-field focal spot from the imaging center for the purpose of background suppression. Also shown was that the symmetric design offers close enough values in field enhancement when compared to the asymmetric design, that the possibility of using it in the current TENOM system is quite real. All in all, the symmetric design, according to the modeling, would offer a reasonable replacement to the current asymmetric design and would theoretically remove a variable from the concerns of the TENOM user.

The concepts used in the LabVIEW VI are sound. They work well for what they are intended. However, there are some bugs which may need to be worked out. For example, when bringing the pattern into view within the FIB software, it is offcenter. This should be corrected in order to make the process more automated and lessen time with the ion beam interacting with the sample. It may also be desirable to expand the size of the on screen pattern as well, however if this is done the number of points in the pattern must remain under 1 million. This means that the pixel density will go down and therefore the quality of the pattern will be lessened. This modification to the VI would allow for higher magnification patterning, allowing for a sharper tip apex. 
For the most part, the etching process has been finalized. Tips are being etched with high reproducibility on the macro level; however, there is no eliminating the variations between different tips in the nanometer regime. This makes for a different initial condition for every tip when beginning the FIB work. However, this is unavoidable; therefore the etching process need not be changed at this time.

One of the motivations for this work turned out to be a detriment; ease of fabrication. This was not an easier probe to manufacture when compared to the asymmetric design, at least not in reality, yet. Once the process has been perfected, it should be an easier process. Depending on the FIB used, rotating the sample to a head on view can be difficult and as mentioned, custom sample holder fabrication may and most likely will, be necessary. Correct parameters for accelerating voltage, beam current and milling times all need to be worked out in detail and a relationship developed between these variables with the etched tip geometry. This will require many hours of work with a FIB system. The old fabrication process is difficult and time consuming but at this point, it works, whereas the lookup table head on method does not currently work. The idea of using bitmaps to create geometries with the FIB works well and could be used in many other applications. However the application of this concept to tip fabrication is still not worked out in full detail.

Why this hasn't worked perfectly is due to a myriad of reasons. For one, grain boundaries present a problem in that different grains mill at different rates. Another reason is that the aspect ratio of each probe is slightly different. Some probes are conical while others are pyramidal. To apply this method to a general case is nontrivial and will require more work. 
The best probe obtained using this method is shown in Figures 5.1 - 5.3. The first figure (5.1) is a low magnification view, showing the whole tip in the head on view. Also visible in this figure is a damaged area of probe from the imaging process in the FIB. This is the $\sim 15 \mu \mathrm{m}$ square sitting in the center of the image. Figure 5.2 is from this damaged region. In this image can be seen at least one grain boundary, which may explain the pyramidal shape on the right side of the tip. Also visible is the tip apex, which is the intended result. This apex is shown in Figure 5.3, from a side, SEM view. As per the scale marker bar, this apex is roughly $400 \mathrm{~nm}$ in diameter, which is too large for good imaging. Generally it is advisable to utilize an apex of 100 nm or less.

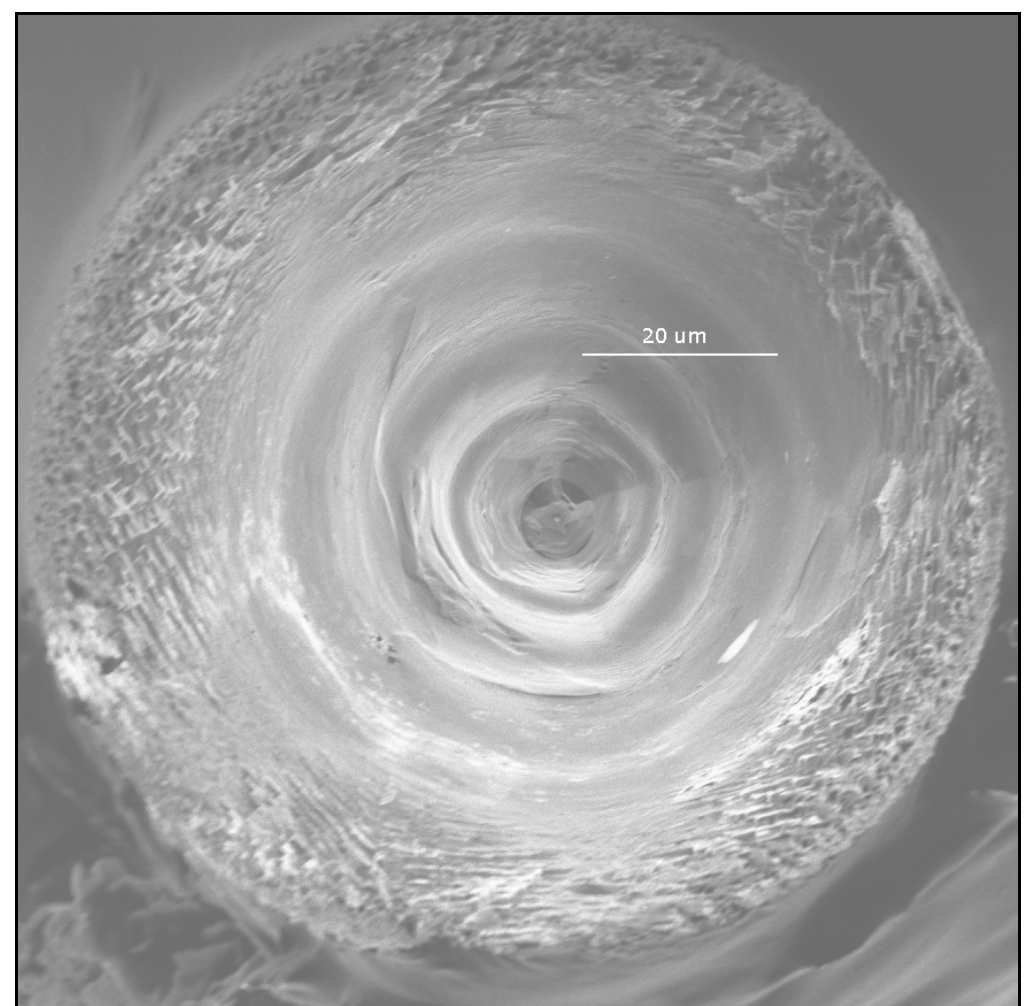

Figure 5.1: Zoomed out head on view of a tip that was fabricated using the lookup table method. 
It has been possible to fabricate a tip apex of less than $100 \mathrm{~nm}$. However, this has only occurred once on a probe that was milled for too long and/or with too high of a beam current (Figure 4.12). It is a proof of concept however and shows that more time need be spent on the FIB portion of this project.

In summary, it appears that the symmetric tip design would indeed work as a near-field probe, however, a non-trivial fabrication method was not found and thus more work needs to be done in this area.

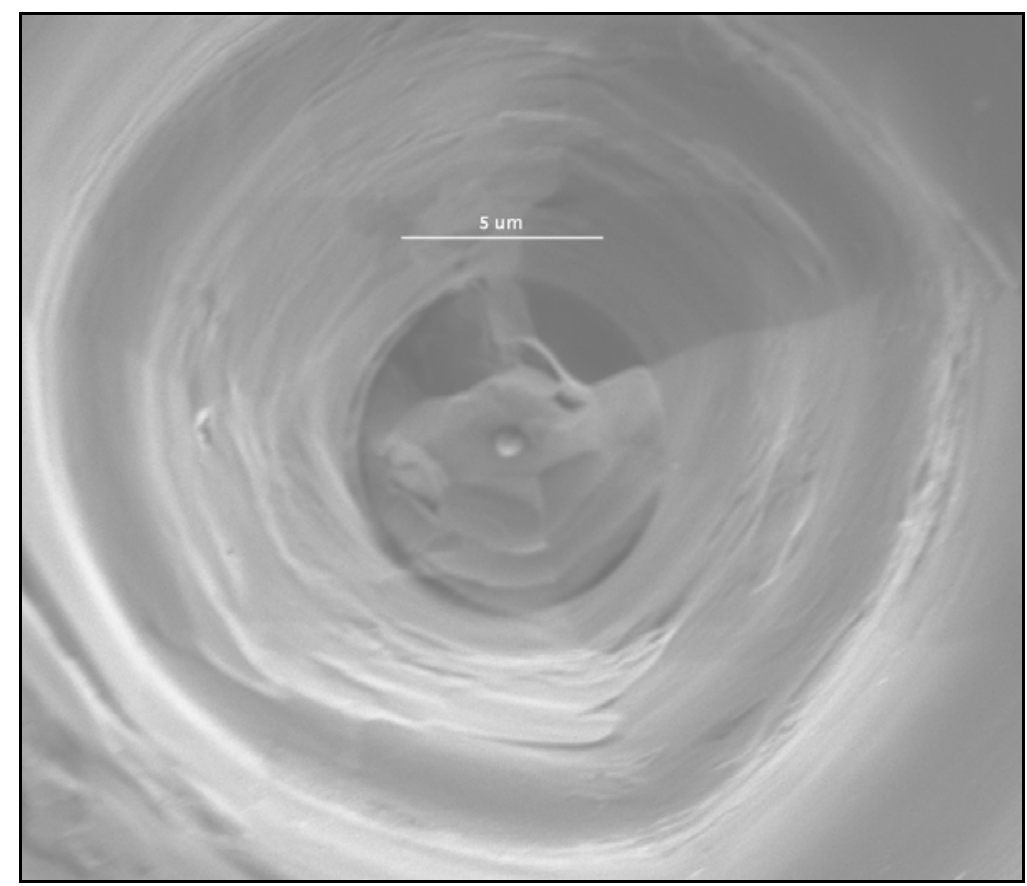

Figure 5.2: A top down view of the best tip created using this lookup table method. Zoomed in view of the damaged square in Figure 5.1.

The next step is to actually mount a symmetric probe and obtain near-field images with it. This step should be straightforward, as the current TENOM system is capable of utilizing the symmetric probe geometry. This would allow for a comparison between the tips in a manner other than numerical modeling.

Another future step is the development alternative probe geometries. Perhaps the symmetric tip is not the best design in terms of field enhancement. Other 
geometries have been shown to produce huge field enhancements (for example, two triangles pointed toward each other on the end of a tip, with a small separation distance). Perhaps this method of fabrication would be beneficial to these designs. Since the method relies only on the bitmaps created, there is no restriction to only symmetric designs.

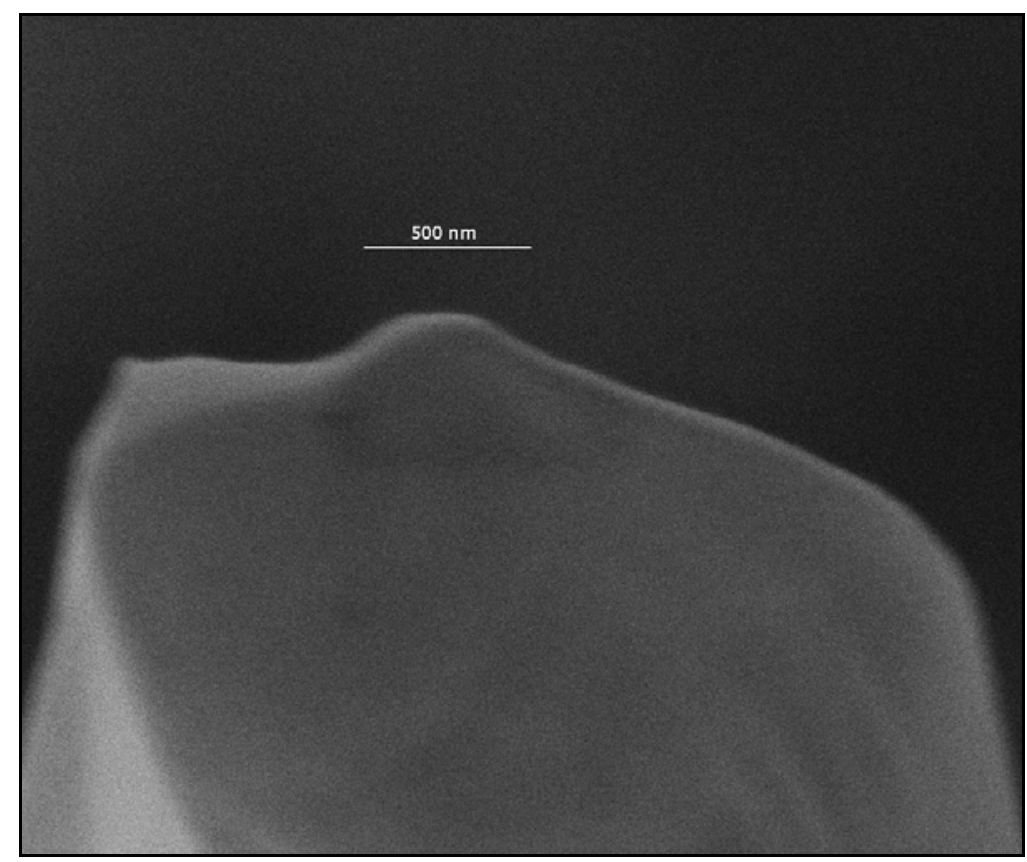

Figure 5.3: A side view of the tip shown in Figures 5.1 and 5.2.

The final step is automation. This would allow for support of a commercialized TENOM system. This step would be non-trivial but very much possible. This could be realized, given an automated, reproducible etching system which places the tip into a specialized holder for the FIB. This holder would have multiple tips at a constant spacing, so that the FIB can move easily from tip to tip. The key would be placing the tips in the holder so that they are all oriented in a consistent manner. This would allow for the FIB to make the same cut and make the same tip in a reproducible way. 


\section{References:}

Ash, E. A. and Nichols, G. (1972). Super-resolution Aperture Scanning Microscope. Nature, Vol. 237, 510-512

Bethe, H. A. (1944). Theory of Diffraction by Small Holes. The Physical Review, Vol 66,163-182

Betzig, E. Isaacson, M. and Lewis, A. (1987). Collection mode nearfield scanning optical microscopy. Applied Physics Letters, Vol 51, 2088-2091

Betzig, E. and Trautman, J. K. (1992). Near-Field Optics: Microscopy, Spectroscopy, and Surface Modification Beyond the Diffraction Limit. Science, Vol. 257, 189-195

Betzig, E. and Chichester, R. (1993). Single Molecules Observed by NearField Scanning Optical Microscopy. Science, Vol 262,1422-1425

Bouwkamp, C. J. (1950). On the diffraction of electromagnetic waves by small circular disks and holes. Philips Research Report

Fotino, M. (1992). Tip Sharpening by normal and reverse electrochemical etching. Rev. Sci. Instr., Vol64, 159-167

Kawata, S. and Inouye, Y. (1993). Scanning probe optical microscopy using a metallic probe tip. Ultramicroscopy, Vol. 57, 313-317

Krug, J., Sanchez, E. J., and Xie, S. Design of near-field optical probes with optimal field enhancement by finite difference time domain electromagnetic simulation, Journal of Chemical Physics, Vol. 116, 10895-10901

Kunz, K. S. and Luebbers, R. (1993). The Finite Difference Time Domain Method for Electromagnetics. New York, NY: CRC Press

Lewis, A. et al. (1984). Development of a 500 angstrom spatial resolution light microscope. Ultramicroscopy, Vol. 13, 227-231

Luebbers, R. J. and Kunz, K. S. (1991). A Frequency-Dependent FiniteDifference Time-Domain Formulation for Transient Propagation in Plasma. IEEE Transactions on Antennas and Propagation, Vol. 39, 29-34

Mehtani, D. et al. (2005). Nano-Raman spectroscopy with side-illumination optics. Journal of Raman Spectroscopy, Vol. 36, 1068-1075 
Novotny, L. and Hecht, B. (2006). Principles of Nano-Optics. Cambridge University Press: New York.

O'Keefe, J. A. (1995). Resolving Power of Visible Light. Journal of the Optical Society of America, Vol. 46, 359.

Pohl, D. W., Denk, W. and Lanz, M. (1984). Optical stethoscopy: Image recording with resolution $\lambda / 20$. Applied Physics Letters, Vol. 44, 651-653

Remcom xFDTD Users manual and Reference Manual. (2005). Version 6.3

Sanchez, E. J., Novotny, L., and Xie, X. S. (1999). Near-Field Fluorescence Microscopy Based on Two-Photon Excitation with Metal Tips. Physical Review Letters, Vol. 82, 4014-4017

Specht, M. et al. (1992). Scanning Plasmon Near-Field Microscope. Physical Review Letters, Vol. 68, 476-479

Synge, E. H. (1928). A Suggested Method for extending Microscopic Resolution into the Ultra-Microscopic Region. Phil. Mag. Vol. 6, 356-362

Wessel, J. (1985). Surface-enhanced optical microscopy. J. Opt. Soc. Am. B, Vol. 2, 1538-1540

Yee, K. S. (1965). Numerical Solution of Initial Boundary Value Problems Involving Maxwell's Equations in Isotropic Media. IEEE Transactions on Antennas and Propagation, Vol.14, 302-307

Zenhausern, F., Martin, Y. and Wickramasinghe, H. K. (1995). Scanning Interferometric Apertureless Microscopy: Optical Imaging at 10 Angstrom Resolution. Science, Vol. 269, 1083-1085 